\title{
The Role of Zooplankton in Regulating Carbon Export and Phytoplankton Community Structure: Integrating Models and Observations
}

\author{
by \\ Kevin Matthew Archibald
}

Submitted to the Department of Earth, Atmospheric, and Planetary Sciences in partial fulfillment of the requirements for the degree of

Doctor of Philosophy

at the

MASSACHUSETTS INSTITUTE OF TECHNOLOGY

and the

WOODS HOLE OCEANOGRAPHIC INSTITUTION

February 2021

(C)2021 Kevin M. Archibald.

All rights reserved.

The author hereby grants to MIT and WHOI permission to reproduce and to distribute publicly paper and electronic copies of this thesis document in whole or in part in any medium now known or hereafter created.

Author

Joint Program in Oceanography/Applied Ocean Science \& Engineering Massachusetts Institute of Technology \& Woods Hole Oceanographic Institution

November 25, 2020

Certified by

Michael G. Neubert

Senior Scientist

Woods Hole Oceanographic Institution

Thesis Supervisor

Certified by

Heidi M. Sosik

Senior Scientist

Woods Hole Oceanographic Institution

Thesis Supervisor

Accepted by . .

Michael Follows

Chair, Joint Committee for Biological Oceanography Massachusetts Institute of Technology 


\title{
The Role of Zooplankton in Regulating Carbon Export and Phytoplankton Community Structure: Integrating Models and Observations
}

\author{
by \\ Kevin Matthew Archibald \\ Submitted to the Department of Earth, Atmospheric, and Planetary Sciences \\ on November 25, 2020, in partial fulfillment of the \\ requirements for the degree of \\ Doctor of Philosophy
}

\begin{abstract}
In this thesis, I explore two topics in plankton ecology with a combination of models and observations. First, I investigate the contribution of zooplankton diel vertical migration (DVM) to the vertical flux of carbon as part of the biological pump. I do this by constructing and analyzing a global model that includes DVM and is driven by satellite-based estimates of primary productivity. There has long been speculation about the significance of DVM to the biological pump, but quantitative estimates of its impact are rare. I estimate that DVM constitutes approximately $16 \%$ of the global carbon export flux associated with the biological pump and that the relative contribution of DVM is higher in subtropical latitudes. In later chapters, I build two nutrient-phytoplanktonzooplankton (NPZ) models with different levels of complexity to evaluate the role of nutrient supply and grazing in promoting phytoplankton diversity. Zooplankton switching plays a significant role in promoting diversity because it allows competing phytoplankton types to coexist in situations that would otherwise lead to competitive exclusion. When implemented in a size-structured NPZ model, stronger switching increases the evenness of the distribution of biomass between coexisting size classes, which is used as a proxy for taxonomic diversity. I also describe a particular characteristic of the Kill-the-Winner functional response (used in the NPZ models), which I have termed synergistic grazing. Synergistic grazing occurs when the grazing rate on one phytoplankton type increases as the biomass of an alternative phytoplankton type increases. This characteristic can result in unintuitive model dynamics. Finally, I describe patterns in phytoplankton community size structure in the shelfbreak region of the Northeast U.S. Shelf using high-resolution flow-cytometry measurements. I find that enhancement of phytoplankton biovolume at the shelfbreak front is common during the springtime, but these enhancement events are not associated with consistent changes in community size structure. I evaluate these results in the context of hypotheses generated based on my analysis of the NPZ models.
\end{abstract}

Thesis Supervisor: Michael G. Neubert

Title: Senior Scientist

Woods Hole Oceanographic Institution

Thesis Supervisor: Heidi M. Sosik

Title: Senior Scientist

Woods Hole Oceanographic Institution 


\section{Acknowledgments}

I would like to thank my committee, Michael Neubert, Heidi Sosik, Stephanie Dutkiewicz, and Dennis McGillicuddy, as well as Scott Doney, for the guidance and feedback they have provided in planning and executing this research. Thank you to Rubao Ji for chairing my thesis defense.

I have been extraordinarily lucky to have had three amazing scientists as advisers during my time at WHOI. Scott, thank you for seeing potential in me and for inspiring me to always think bigger. Mike, thank you for your kind and thoughtful advice and for always looking out for my well-being, both in and out of the lab. Heidi, thank you for providing me with so many wonderful research opportunities and for all they ways you have encouraged me to never stop striving for excellence.

To the other members of both the Doney and Sosik lab groups, thank you for all the mentorship, assistance, and camaraderie. My thesis is much improved as a result of the great work environments I got to be a part of, both in terms of scientific excellence and a friendly, accepting atmosphere.

To all those involved in the SPRIOPA project, thank you for helping me navigate the complex, interdisciplinary questions that always seem to be popping up when I am trying to interpet my results. Thank you also for making many sea-sick cruises a little more fun.

Many thanks as well to the academic programs offices at WHOI and at MIT for all the ways in which they have supported me as a student and as a researcher.

Finally, to my dear friends and family, thank you for the endless and unconditional support you have given me over the years. I am forever grateful.

The research in this thesis was supported by the National Science Foundation (NSF, OCE-1434000 and OCE-1657803) and the National Aeronautics and Space Administration (NASA) as part of the EXport Processes in the global Ocean from RemoTe Sensing (EXPORTS) field campaign (grant 80NSSC17K0692) and the North Atlantic Aerosol and MarineEcosystems Study (NAAMES, grantsNNX15AE72G and 80NSSC18K0018). Additional support came from NSF (OCE-1655686 and OCE-1657803) and the Simons Foundation (561126). 


\section{Contents}

1 Introduction $\quad 15$

2 Modeling the impact of zooplankton diel vertical migration on the carbon export

$\begin{array}{ll}\text { flux of the biological pump } & 21\end{array}$

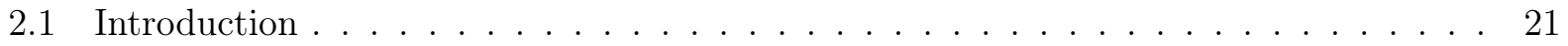

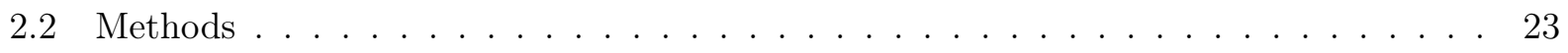

2.2.1 Model Construction . . . . . . . . . . . . . . . . . . . 23

2.2.2 Application of the Model . . . . . . . . . . . . . . . . . . . 34

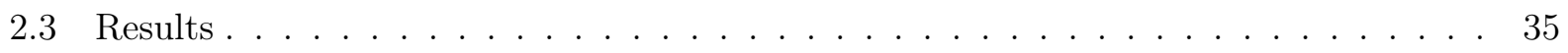

2.3 .1 1-D Model at BATS . . . . . . . . . . . . . . . . 35

2.3.2 Global Model Dynamics . . . . . . . . . . . . . . . . . . . . 37

2.3.3 Model Sensitivity . . . . . . . . . . . . . . . . . . . 40

2.4 Discussion . . . . . . . . . . . . . . . . . . . . . . . 45

3 The Kill-the-Winner functional response: Coexistence dynamics and synergistic $\begin{array}{ll}\text { grazing } & 57\end{array}$

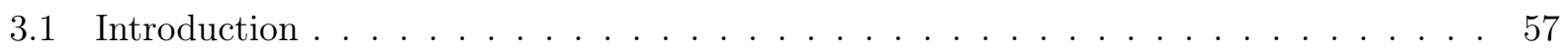

3.2 Model Construction and Analysis . . . . . . . . . . . . . . . . . . 59

3.2.1 Preference and switching in the KTW functional response . . . . . . . . . . 59

3.2.2 The diamond-shaped food web model . . . . . . . . . . . . . . 61

3.3 Discussion . . . . . . . . . . . . . . . . . . . . . . 71

4 Increased nutrient supply and zooplankton switching increase diversity in a size- 


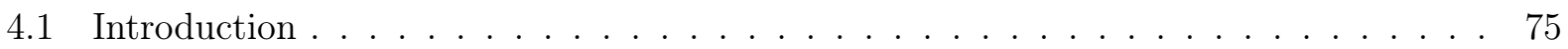

4.2 Model Construction and Simulation . . . . . . . . . . . . . . . . 77

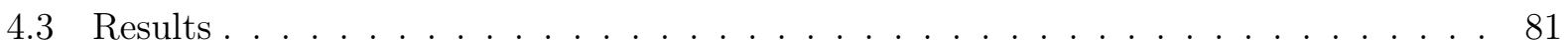

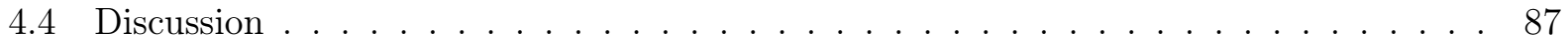

5 Patterns in phytoplankton community size structure in the shelfbreak front re$\begin{array}{ll}\text { gion of the Northeast U.S. Shelf } & 91\end{array}$

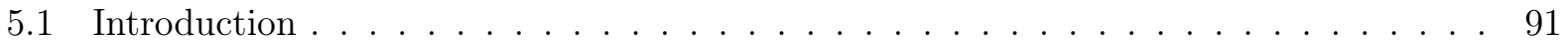

5.2 Methods . . . . . . . . . . . . . . . . . . . . . . 95

5.2 .1 Underway instrument setup . . . . . . . . . . . . . . . . . . . 95

5.2 .2 Data calibration and collation . . . . . . . . . . . . . . 97

5.2 .3 Categorical variables . . . . . . . . . . . . . . . . . . . . . . 98

5.2 .4 Statistical analyses . . . . . . . . . . . . . . . . . . . 99

5.3 Results . . . . . . . . . . . . . . . . . . . . . . 101

5.3 .1 Hydrographic context . . . . . . . . . . . . . . . 101

5.3.2 Patterns in total phytoplankton biovolume . . . . . . . . . . . . . . 105

5.3 .3 Patterns in size structure f . . . . . . . . . . . . . . . 107

5.4 Discussion . . . . . . . . . . . . . . . . . . . . . . 115

6 Conclusions $\quad 121$

A Proof of competitive exclusion of phytoplankton $P_{2}$ under Cases 1 and 3 of the diamond-shaped food web model $\quad 125$

$\begin{array}{lr}\text { B References } & 127\end{array}$ 


\section{List of Figures}

2-1 Conceptual diagram of the plankton food web as represented in the model and including the various export fluxes. Biomass standing stocks are indicated by solid lines and fluxes by dashed lines. Orange indicates a passive sinking export flux out of the euphotic zone and purple indicates DVM-mediated fluxes. The gray dashed line shows values that are empirical observations derived from satellite data products. 28

2-2 (a) Biomass of nano- and micro-phytoplankton $\left(\mathrm{P}_{n}, \mathrm{P}_{m}\right)$ and zooplankton $(\mathrm{Z})$ in the mixed layer over the seasonal cycle at the Bermuda Atlantic Time Series grid point. (b) Depth of the euphotic zone $\left(\mathrm{z}_{e u}\right)$, the mixed layer $\left(\mathrm{z}_{m l}\right)$, and the $10^{-3} \mathrm{~W} / \mathrm{m}^{2}$

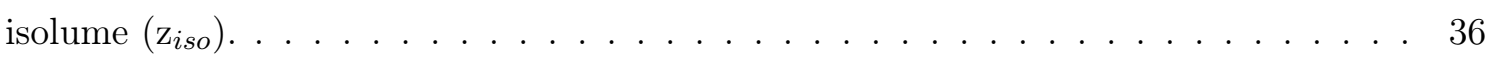

2-3 Monthly means of (a) the passive sinking flux $\left(F_{\text {eu }}\right)$, (b) the DVM-mediated export flux $\left(J_{d v m}\right),(\mathrm{c})$ the export ratio $(\mathrm{ER})$, DVM export ratio (DER), and the DVM respiration ratio (DRR) and (d) respiration depression (RD) at BATS. Note the difference of vertical scales between $(\mathrm{a})$ and $(\mathrm{b}) \ldots \ldots \ldots \ldots \ldots$

2-4 Vertical profiles at BATS of (a) particulate carbon flux (F(z)) and (b) the production of DIC $(R(z))$ in the twilight zone for the month of April, during the peak of the phytoplankton bloom. The euphotic zone is indicated by the gray box. The blue line represents a model run when $p_{d v m}=0.5$ and the red dashed line represents the control run when $p_{d v m}=0$.

2-5 Global distribution of (a) net primary productivity (NPP), (b) the NPP biovolume ratio $\left(\mathrm{NPP}_{n} / \mathrm{NPP}_{m}\right),(\mathrm{c})$ zooplankton biomass $(\mathrm{Z})$, and (d) migration depth $\left(\mathrm{z}_{\text {iso }}\right)$.

The value at each grid point is the annual mean over the seasonal cycle. . . . . . . . 41 
2-6 Global distribution of the magnitude of the (a) total export flux, (b) the passive sinking export flux $\left(\mathrm{F}_{e u}\right)$, and (c) the DVM-mediated export flux $\left(\mathrm{J}_{d v m}\right)$. The value at each grid point is the annual mean over the seasonal cycle. . . . . . . . . . . . 43

2-7 Global distribution of the (a) export ratio (ER), (b) DVM export ratio (DER), (c) DVM respiration ratio (DRR), and (d) respiration depression $(\mathrm{RD})$. The value at each grid point is the annual mean over the seasonal cycle. . . . . . . . . . . . . 44

2-8 Results of the sensitivity analysis. Each box represents the percent change in the model output based on a $1 \%$ increase in the parameter value. Parameter rows with zero sensitivity on all the output metrics have been excluded. . . . . . . . . . . . 46

2-9 Comparison of model estimates of the DVM-mediated export flux to empirical observations at a variety of locations. A 1:1 line is included for reference. . . . . . . . 50

3-1 The proportion of $P_{1}$ in a zooplankter's diet as a function of the proportion of $P_{1}$ in the environment based on Eq. 3.1 and assuming two phytoplankton types. The different curves show different combinations of preference and switching: (a) neither preference nor switching $\left(\rho_{1}=\rho_{2}, \alpha=1\right)$, (b) switching without preference $\left(\rho_{1}=\rho_{2}, \alpha>1\right)$, (c) preference for $P_{1}$ without switching $\left(\rho_{1}>\rho_{2}, \alpha=1\right)$, (d) preference for $P_{1}$ with switching $\left(\rho_{1}>\rho_{2}, \alpha>1\right)$, (e) preference for $P_{2}$ without switching $\left(\rho_{1}<\rho_{2}, \alpha=1\right)$, (f) preference for $P_{2}$ with switching $\left(\rho_{1}<\rho_{2}, \alpha>1\right) \ldots \ldots \ldots$

3-2 (a) The structure of the diamond-shaped food web model and (b) the MichaelisMenten curves for the nutrient uptake of $P_{1}$ and $P_{2} \ldots \ldots \ldots$

3-3 Asymptotic behavior of Cases 2 and 4-6 of model (3.2)-(3.4) as a function of $N_{0}$ and $D$. For each parameter combination we chose initial conditions at random from uniform distributions $\left(N(0) \sim U[0,15], P_{1}(0) \sim U[0,2], P_{2}(0) \sim U[0,2], Z(0) \sim U[0,10]\right)$, and simulated the model from $t=0$ to $t=10,000$ days. Colors indicate the asymptotic result. Along the black curve $r_{2}=0$ (cf., Eq 3.9); below the curve, $r_{2}<0$ and above the line $r_{2}>0$. A fifth outcome, in which $N=N_{0}$ and $P_{1}=P_{2}=Z=0$, is not illustrated in this graph. When $N_{0}<R_{1}^{*}$, this "washout equilibrium" is stable. The washout region is too small to be seen in the graphs above. . . . . . . . . . . 68 
3-4 $G_{1}\left(1, P_{2}\right)$ is the grazing rate on $P_{1}$ as a function of the density of $P_{2}$, when $P_{1}=1$. In these graphs, synergistic grazing occurs when $\partial G_{1}\left(1, P_{2}\right) / \partial P_{2}>0$; i.e., when the zooplankter exhibits switching behavior $(\alpha=2)$. Other parameters are given in

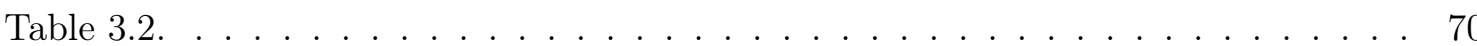

4-1 Size-structured model configuration with one nutrient (N) box, $n$ phytoplankton boxes $(\mathrm{P})$, and one zooplankton box $(\mathrm{Z}) \ldots \ldots \ldots \ldots \ldots$

4-2 Total phytoplankton community biomass and zooplankton biomass at equilibrium, as well as phytoplankton size class richness, as a function of input nutrient concentration $\left(N_{0}\right)$ under both $(\mathrm{a})$ non-switching and $(\mathrm{b}-\mathrm{d})$ switching scenarios. $\ldots \ldots \ldots . . . .83$

4-3 Distribution of biomass between the 10 phytoplankton size classes at equilibrium for different values of $\alpha$ as a function of $N_{0} \ldots \ldots \ldots \ldots \ldots$

4-4 Phytoplankton community size class (a) richness, (b) evenness, and (c) Shannon Diversity as a function of $N_{0}$ and $\alpha$. The contours show $r_{j}=0$ for each size classes $j=1: 10$. For these simulations, $k_{\text {sat }}=15$.

4-5 Phytoplankton community size class (a) richness, (b) evenness, and (c) Shannon Diversity as a function of $N_{0}$ and $k_{\text {sat }}$. The contours show $r_{j}=0$ for each size classes $j=1: 10$. The vertical axis is plotted in the reverse direction because smaller values of $k_{\text {sat }}$ correspond to stronger zooplankton grazing pressure. For these simulations,

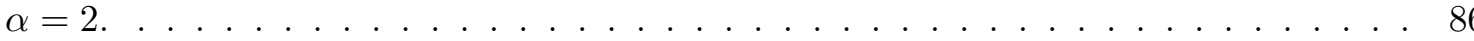

5-1 A conceptual model demonstrating how increased phytoplankton size class evenness may be generated under conditions of simultaneous high nutrient availability and high zooplankton grazing impact. . . . . . . . . . . . . . . . . 94

5-2 Location of the SPIROPA sampling transect (black) and the ship tracks for each of the three cruises. Bathymetry is shown in gray. . . . . . . . . . . . . . . . 96

5-3 Composite particle size distributions for each of the three cruises. . . . . . . . . 102

5-4 Time series for each cruise showing the total biovolume concentration and biovolume in each size class. Data are plotted on the same y-axis for each cruise. . . . . . . . 103 
5-5 Time series for each cruise showing the total biovolume concentration and biovolume in each size class. Data are plotted using different y-axes for each cruise to better visualize variability within the time series. . . . . . . . . . . . . . . 104

5-6 AVHRR satellite imagery showing sea surface temperature over the study area during (a) early SPR18, (b) late SPR18, (c) SPR19, and (d) SUM19. Transect station locations are indicated with black circles. . . . . . . . . . . . 106

5-7 Distribution of total biovolume concentration in each water mass for all seasonal

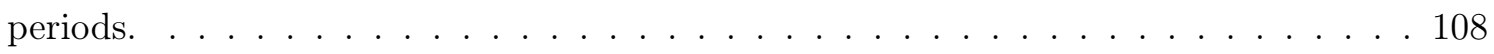

5-8 Simplex plots showing biovolume fraction of each size class. Samples are color coded by water mass. . . . . . . . . . . . . . . . . . . . . . 109

5-9 Distribution of picoplankton biovolume fraction for each water mass for all seasonal periods. Note that range of the $\mathrm{x}$-axis is limited to $(0,0.3) \ldots \ldots \ldots$

5-10 Distribution of nanoplankton biovolume fraction for each water mass for all seasonal

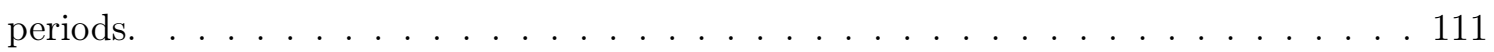

5-11 Distribution of microplankton biovolume fraction for each water mass for all seasonal

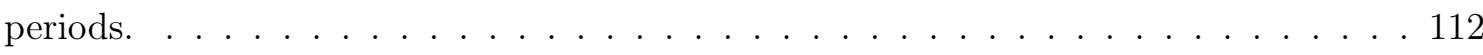

5-12 Distribution of biovolume concentrations in each size class, and the resulting mean size class composition, in each water mass. . . . . . . . . . . . 113

5-13 Distribution of Shannon Diversity Index and Evenness calculated from total biovolume in each size class. . . . . . . . . . . . . . . . . . . . . . 114 


\section{List of Tables}

2.1 DVM model state variables . . . . . . . . . . . . . . . . . 25

2.2 DVM model parameters . . . . . . . . . . . . . . . . . . . . . 33

2.3 Summary of global statistics. Annual mean and standard deviation values include temporal variability across months in the yearly climatology and spatial variability across the global model domain. . . . . . . . . . . . . . . . . . . . 42

2.4 Summary of the comparisons between model output at BATS and in situ observations made at the Bermuda Atlantic Time Series scientific station. Observation means and standard deviations (SD) are taken from reviews by (Steinberg et al., 2001) and (Madin et al., 2001). . . . . . . . . . . . . . . . . . . 4 48

3.1 Summary of model test cases in the diamond-shaped food web model ${ }^{a, b} \ldots 66$

3.2 Parameters in the diamond-shaped food web model . . . . . . . . . . . . . 67

4.1 Parameter values used to simulate the size-structured phytoplankton community model 80

5.1 Date ranges and abbreviations for the four seasonal periods over the SPIROPA project.100

5.2 Summary of statistically significant differences in water mass characteristics for each seasonal period. The second column lists which water masses had significantly higher biovolume concentrations compared to all other water masses for that period. Columns three through five indicate whether those water masses with higher biovolume concentration also had significantly different size class composition, Shannon Diversity Index, and Evenness. . . . . . . . . . . . . . . 116 


\section{Chapter 1}

\section{Introduction}

Plankton are ubiquitous in the world's oceans. Phytoplankton are responsible for roughly one-half of the planet's primary productivity (Field et al., 1998). The most common zooplankton, copepods, are the most abundant multicellular animals on earth (Schminke, 2007). The coordinated vertical migrations of zooplankton have been described as the largest migration events anywhere on earth (Hays, 2003). Plankton ecosystems form the base of the entire marine food web, supporting higher trophic levels and constraining global fisheries catch (Chassot et al., 2010). Phytoplankton and zooplankton are also critically important for cycling nutrients on a global scale and help to regulate the climate (Buitenhuis et al., 2006; Sabine et al., 2004). Pursuing a deeper understanding of the complex dynamics of this diverse group of organisms has inspired oceanographers for more than a century, and the use of ecological models has, historically, played an important role in that pursuit.

A model is a set of one or more equations that describe the behavior of an ecological system. Models in oceanography range from the very simple, such as a single equation describing the growth of phytoplankton during the spring bloom (Fleming, 1939), to the highly complex, such as the Biogeochemical Elemental Cycling (BEC) component of the Community Earth System Model (CESM), which includes dozens of state variables and hundreds of parameters (Letscher et al., 2015). Models are a fundamental tool in oceanography. The utility of models can be characterized by examining the three types of questions that models help answer: theoretical, heuristic, and predictive (Franks, 2002). That is, when a researcher asks the question, "What would happen?", "How does it happen?", or "What will happen?", then a model can help answer that question by abstracting the relevant ecological processes and simulating them under a variety of assumptions or conditions. 
One kind of model is especially important to this thesis: the Nutrient-Phytoplankton-Zooplankton (NPZ) model. The use of NPZ models in oceanography was pioneered by Gordon Riley, who developed a model of the seasonal phytoplankton dynamics on Georges Bank, and area in the Gulf of Maine near Cape Cod, MA (Riley, 1946). Riley's model was surprisingly simple, including only six parameters and six environmental forcing variables. Nevertheless, his model was able to reproduce the dynamics of the spring bloom quite well. More importantly, by describing the spring bloom in terms of the balance between phytoplankton growth and loss due to respiration and grazing, Riley demonstrated the utility of models for describing and understanding the drivers behind ecological phenomena.

In the decades since Riley's foundational study, models of planktonic marine ecosystems have expanded rapidly in both complexity and scope (Anderson and Gentleman, 2012). Detritus was identified as a biogeochemically important resource in marine ecosystems and modelers began building NPZD models that included the recycling and export of organic material in the environment (Fasham et al., 1990; Steele, 1974; Wroblewski, 1977). Others noted the vast diversity in phytoplankton biogeochemical function and have expanded the phytoplankton component of NPZ models to include different phytoplankton functional types (Gregg et al., 2003; Le Quéré et al., 2005; Moore et al., 2002). Cell size was also identified as a particularly important factor for structuring phytoplankton communities since many biogeochemical characteristics of phytoplankton are related to their size (Poulin and Franks, 2010; Sieburth et al., 1978; Ward et al., 2012). Researchers began studying the effect of physical dynamics on plankton communities by coupling NPZ models to ocean hydrographic models to study the dynamics of plankton ecosystems in the context of their physical environment (Evans and Parslow, 1985; Flierl and Davis, 1993; Franks and Chen, 2000; McGillicuddy et al., 1995a; McGillicuddy et al., 1995b). The most advanced modern biogeochemical models simulate marine primary productivity on a global scale with nutrient, phytoplankton, and zooplankton components containing explicit representations of many different, biogeochemically unique functional types coupled to complex global ocean and atmospheric circulation models (Aumont et al., 2015; Letscher et al., 2015; Thorton et al., 2009).

The availability of sophisticated and highly complex models raises an important question - do simple NPZ models have any place in modern biological oceanography, or should they be viewed as precursors to more advanced models better suited to represent our increasing understanding of 
the complex dynamics of marine plankton? I argue that the advantages of simple models lie in the simplicity itself. Simple models have a number of practical advantages over more complicated ones. The small number of parameters and state variables means that analytical methods for studying their dynamic behavior are often available. The range of simulation scenarios are more easily explored and the output is generally more easily understood. Sensitivity analysis is also more feasible to conduct and interpret.

Beyond the practical advantages however, there are certain kinds of questions for which simple models are better suited. A simple model, when well-designed and applied in the appropriate context, is a powerful tool to abstract specific ecological interactions and examine their dynamics. Simple models describe the fundamental drivers and mechanisms within a system. As such, they are a logical starting point when approaching an ecological question. A simple model should not be rejected unless it fails to adequately describe the relevant ecological phenomena. Analysis should therefore progress first from an understanding of a simple model on to the analysis of more complex models, as the need arises given the question being addressed.

This is not in any way to say that more complex models are not appropriate for some questions. The choice of model should be informed by the question. There is a famous quote from the statistician George Box, "All models are wrong, but some are useful" (Box, 1979). I remind myself of this often. Because every model is, to some degree, an abstraction, the purpose of a model is never to perfectly reproduce the details of an ecosystem in silico, but rather to tell us something interesting about an ecosystem. For some questions, a complex global biogeochemical model is necessary, and for some questions, such as those explored in this thesis, a humble NPZ model is exactly what we need.

The use of models, regardless of their complexity, comes with a unique set of challenges. Since models are abstractions of real ecosystems, the assumptions used in the model often do not hold true in the real world. This makes comparing model simulations to observational data a potentially complicated task. Inevitably, data collected in the field is subject to processes and variability not represented in the model. Working with both models and field data, therefore, is an iterative process. That is, the model helps us to interpret the data and the data, in turn, helps constrain the construction of the model. Theoretical models help us generate hypotheses about how we think an ecosystem works. We can then test these hypotheses by making observations and conducting 
experiments. By comparing our model against the observations, we can learn where our model fails to represent the ecosystem and ask how the model can be improved.

This thesis contains two examples of questions that can be investigated through the collaborative use of simple models and field data. In Chapter 2, I ask what contribution zooplankton diel vertical migration (DVM) makes to the global carbon export flux of the biological pump. I build an NPZ model to represent the surface plankton ecosystem and describe two types of fluxes of carbon out of the surface ocean - the passive sinking flux of particulate organic carbon and the DVM-mediated flux associated with zooplankton making daily migrations from the surface to the twilight zone. This model is forced by satellite measurements of primary productivity and the size structure of the phytoplankton community. I use model simulations to characterize the global variability in these two fluxes and investigate what factors control the relative contribution of zooplankton DVM to carbon export. The model is validated using empirical measurements of the export flux in a variety of biogeochemical regimes and historical data of the global distribution of zooplankton biomass.

In Chapters 3-5, I explore how bottom-up and top-down controls on phytoplankton communities promote diversity. I build two different NPZ models to investigate different aspects of this relationship. In Chapter 3, I use a diamond-shaped food web model to evaluate zooplankton switching, a phenomenon in which zooplankton grazers modify their feeding behavior based on the relative abundance of different phytoplankton types in the environment. Switching allows two competing phytoplankton types to coexist in situations that would otherwise lead to competitive exclusion. In Chapter 4, I expand the diamond-shaped food web model to a size-structured phytoplankton community model and investigate how the coexistence criteria play out in a community with multiple competing phytoplankton size classes. Using the distribution of phytoplankton biomass between size classes as a proxy for diversity, I explore how the combination of high nutrient availability and zooplankton switching results in increased diversity in the phytoplankton community. In Chapter 5, I analyze data collected during multiple cruises across the Northeast U.S. continental shelf and describe patterns in phytoplankton community size structure and its relationship to frontal enhancement in phytoplankton biomass. I leverage the understanding gained through the analysis of NPZ models in previous chapters to generate hypotheses about the mechanisms that control size structure in the region.

The combination of NPZ models and observational data presented in this thesis contributes to 
our understanding of the important role that zooplankton play in pelagic ecosystems. Zooplankton are much more than just a link that connects primary production to higher trophic levels. By modulating the competition between multiple phytoplankton types, zooplankton grazing helps generate the dazzling array of diversity observed in marine phytoplankton communities. Zooplankton also have important biogeochemical functions of their own, and are a critical pathway of carbon export in the biological pump. By representing these phenomena in simple NPZ models, I was able to isolate and analyze a few specific mechanisms that make zooplankton so important in biological oceanography. I hope that the work presented here encourages others to appreciate what an important tool NPZ models have, and continue to be, in the pursuit of understanding our world's oceans. 


\section{Chapter 2}

\section{Modeling the impact of zooplankton diel vertical migration on the carbon export flux of the biological pump}

This chapter was originally published as: Archibald, K. M., Siegel, D. A., and Doney, S. C. (2019). Modeling the impact of zooplankton diel vertical migration on the carbon export flux of the biological pump. Global Biogeochemical Cycles, 33(2), 181-199.

This publication is reproduced here with permission from the publisher, The American Geophysical Union.

\section{$2.1 \quad$ Introduction}

Marine food webs help mediate the transfer of atmospheric carbon dioxide downward into the water

column via a suite of ecological mechanisms collectively referred to as the biological pump (Falkowski et al., 1998). Photosynthesis by marine phytoplankton fixes carbon dioxide in the ocean's surface into organic carbon, which is passed through the food web. A portion of this carbon sinks out of the euphotic zone in the form of algal cell aggregates and zooplankton fecal pellets, or may be actively transported to depth by zooplankton vertical migrations (Steinberg et al., 2000). The integrated effect of these mechanisms is a downward flux of carbon from the surface ocean deeper into the water 
column, where carbon may be sequestered in the deep ocean on time scales that are significant for ecological and climate processes (Falkowski et al., 1998).

Quantifying the carbon export flux of the biological pump and understanding how it may change in response to variable climate or biogeochemical conditions is critical to gaining a complete and predictive understanding of the global carbon cycle (Siegel et al., 2014). Since comprehensive global studies of the biological pump are financially and logistically challenging, the development of mechanistic models is an important supplement to field studies. A key step in the development of these models is exploring the impact of different pathways of carbon export (Siegel et al., 2016). In addition to particles that sink out of the euphotic zone, carbon can also be actively transported by the diel vertical migrations of zooplankton (Siegel et al., 2016; Steinberg and Landry, 2017).

Many species of zooplankton participate in diel vertical migrations (DVM), spending the dark hours of the night in surface waters to graze on phytoplankton and smaller zooplankton, then migrating to depth during the day and simultaneously transporting grazed carbon with them (Longhurst et al., 1990; Steinberg and Landry, 2017). This behavior may be a response intended to reduce predation by visual predators in the well-lit surface waters during the daylight hours (Hays, 2003; Lampert, 1989). A portion of the carbon that is grazed at the surface during the night is metabolized or deposited as fecal pellets in the twilight zone during the day, thereby contributing to the export of carbon to below the euphotic zone (Steinberg et al., 2002).

A number of modeling studies have quantified the regional contribution of zooplankton DVM to the carbon export flux in a variety of environments, and consistently found a significant effect of DVM on the biological pump (Bianchi et al., 2013b; Putzeys and Hernández-Leon, 2005; Wallace et al., 2013). Work by (Bianchi et al., 2013b) concluded that carbon transported via DVM accounts for $10-30 \%$ of the total particulate flux and as much as $50 \%$ of the metabolic carbon dioxide produced in the twilight zone. These results underscore the importance of spatially decoupling grazing from zooplankton respiration and the production of fecal pellets and demonstrates the strong influence that DVM can have on mesopelagic biogeochemistry (Bianchi et al., 2013a). Still, the DVM transport pathway remains an uncommon feature in biogeochemical models of carbon export and the global contribution of DVM-mediated export is largely unquantified.

This study extends the work of (Siegel et al., 2014) by quantifying the contribution of DVM activity to the total export flux of the biological pump and exploring the effect of DVM on mesopelagic 
biogeochemistry. We apply the model to a global domain to compare how variable forcing conditions change how DVM contributes to the biological pump. Because the underlying food-web model is driven diagnostically with satellite ocean color data, the expanded euphotic zone-mesopelagic model provides capability to estimate time-evolving DVM globally over seasonal cycles, as well as estimate inter-annual variability. The model is introductory in nature and we make a number of simplifying assumptions concerning zooplankton population dynamics and DVM behavior in order to explore the broad-scale implications of DVM-mediated export and the effects on twilight zone biogeochemistry. Our hope is that this model will contribute to a mechanistic understanding of the role of zooplankton populations in the biological pump.

\subsection{Methods}

\subsubsection{Model Construction}

The model is constructed using two components - the existing euphotic zone module (Siegel et al., 2014) and a new twilight zone (mesopelagic) module. The euphotic zone component uses a food web model forced by satellite observations of net primary production (NPP) and the time rate of change in phytoplankton biomass and size structure to estimate export of both algal aggregates and zooplankton fecal pellets out of the surface ocean. For details on the satellite data products used to drive the surface module, see (Siegel et al., 2014). The twilight zone module tracks the fate of exported carbon in the mesopelagic and adds the effects of zooplankton DVM. The twilight zone component is vertically structured and defines the depth dependence of the vertical flux of particulate organic carbon (POC) and the production of respiratory dissolved inorganic carbon (DIC), or equivalently, oxygen consumption.

Export out of the euphotic zone is modeled here as the sum of two separate fluxes, the passive sinking flux $\left(F_{e u}\right)$ and the DVM-mediated flux $\left(J_{d v m}\right)$.

$$
\text { Total Export Flux }=F_{e u}+J_{d v m}
$$

$F_{\text {eu }}$ is defined as the sum of the sinking of phytoplankton cell aggregates $\left(F_{\text {alg }}\right)$ and the sinking of 
zooplankton fecal pellets produced in the euphotic zone $\left(F_{f e c}\right)$.

$$
F_{e u}=F_{a l g}+F_{f e c}
$$

$J_{d v m}$ is the sum of fecal pellets produced in the twilight zone $\left(J_{f e c}\right)$ and carbon metabolized by migrating zooplankton in the twilight zone $\left(J_{m e t}\right)$.

$$
J_{d v m}=J_{f e c}+J_{m e t}
$$

\section{The Euphotic Zone Module}

The drivers of the food web model in the euphotic zone are satellite-derived monthly climatologies of NPP and phytoplankton biomass in two size classes, nano- and micro-phytoplankton $\left(P_{n}, P_{m}\right)$, from

the Sea-viewing Wide-Field-of-view Sensor (SeaWiFS) ocean color mission (Siegel et al., 2014). The model assumes that a fixed proportion $\left(f_{\text {alg }}\right)$ of the NPP fraction produced by micro-phytoplankton integrated over the euphotic zone $\left(\mathrm{NPP}_{m}\right)$ is exported as sinking aggregates such that

$$
F_{\text {alg }}=f_{\text {alg }} N P P_{m}
$$

None of the NPP fraction from nano-phytoplankton $\left(\mathrm{NPP}_{n}\right)$ is exported as sinking aggregates. The value of $f_{a l g}$ is assumed to be 0.1 based on previous food web models (Boyd and Trull, 2007; Michaels and Silver, 1988; Siegel et al., 2014). Fecal pellet production depends on zooplankton grazing, where the volumetric rate of grazing on each phytoplankton class $\left(G_{n}, G_{m}\right)$ is estimated using a food web model forced by measurements of NPP and the time rate of change in phytoplankton biomass (Siegel et al., 2014).

$$
\frac{d P_{i}}{d t}=\frac{N P P_{i}}{z_{e u}}-G_{i}-\delta_{i} \frac{F_{a l g}}{z_{e u}}-m_{p} P_{i}-\frac{P_{i}}{z_{m l}} \frac{d z_{m l}}{d t} H\left(\frac{d z_{m l}}{d t}\right)
$$

$\mathrm{dP}_{i} / \mathrm{dt}$ is the observed time rate of change of the ith size fraction of phytoplankton biomass, $\mathrm{NPP}_{i}$ is the vertically integrated net primary production of each phytoplankton size fraction, $G_{i}$ is the grazing rate on each size fraction of phytoplankton, $z_{e u}$ is the euphotic depth, and $m_{p}$ is the linear mortality rate of phytoplankton. The phytoplankton linear mortality rate is equal to $0.1 \mathrm{~d}^{-1}$ 
Table 2.1: DVM model state variables

\begin{tabular}{||c|c|c||}
\hline Variable & Description & $\mathrm{Units}$ \\
\hline$F_{e u}$ & total POC flux out of the euphotic zone & $\mathrm{mgC} / \mathrm{m}^{2} \mathrm{~d}$ \\
$J_{d v m}$ & DVM-mediated export flux & $\mathrm{mgC} / \mathrm{m}^{2} \mathrm{~d}$ \\
$F_{a l g}$ & flux of algal aggregates out of the euphotic zone & $\mathrm{mgC} / \mathrm{m}^{2} \mathrm{~d}$ \\
$F_{f e c}$ & flux of fecal pellets out of the euphotic zone & $\mathrm{mgC} / \mathrm{m}^{2} \mathrm{~d}$ \\
$J_{f e c}$ & flux of fecal pellets produced in twilight zone & $\mathrm{mgC} / \mathrm{m}^{2} \mathrm{~d}$ \\
$J_{m e t}$ & metabolized carbon produced in twilight zone & $\mathrm{mgC} / \mathrm{m}^{2} \mathrm{~d}$ \\
$P_{n}$ & nano-phytoplankton biomass & $\mathrm{mgC} / \mathrm{m}^{3}$ \\
$P_{m}$ & micro-phytoplankton biomass & $\mathrm{mgC} / \mathrm{m}^{3}$ \\
$N P P_{m}$ & micro-phytoplankton NPP & $\mathrm{mgC} / \mathrm{m}^{2} \mathrm{~d}$ \\
$N P P_{n}$ & nano-phytoplankton NPP & $\mathrm{mgC} / \mathrm{m}^{2} \mathrm{~d}$ \\
$G_{m}$ & grazing rate on micro-phytoplankton & $\mathrm{mgC} / \mathrm{m}^{3} \mathrm{~d}$ \\
$G_{n}$ & grazing rate on nano-phytoplankton & $\mathrm{mgC} / \mathrm{m}^{3} \mathrm{~d}$ \\
$z_{e u}$ & depth of the euphotic zone & $\mathrm{m}$ \\
$z_{m l}$ & depth of the mixed layer & $\mathrm{m}$ \\
$G_{a b s}$ & grazed carbon absorbed by zooplankton & $\mathrm{mgC} / \mathrm{m}^{3} \mathrm{~d}$ \\
$Z$ & zooplankton biomass & $\mathrm{mgC} / \mathrm{m}^{3}$ \\
$z_{i s o}$ & depth of the $10^{-3}$ W $/ \mathrm{m}^{2}$ isolume & $\mathrm{m}$ \\
$F(z)$ & POC flux in the twilight zone & $\mathrm{mgC} / \mathrm{m}^{3} \mathrm{~d}$ \\
$T(z)$ & temperature & ${ }^{\circ} \mathrm{C}$ \\
$O_{2}(z)$ & dissolved oxygen concentration & $\mu \mathrm{mol} / \mathrm{L}$ \\
$R(z)$ & time rate of change of DIC in the twilight zone & $\mathrm{mgC} / \mathrm{m}^{3} \mathrm{~d}$ \\
\hline
\end{tabular}


following previous model parameterizations (Moore et al., 2004; Siegel et al., 2014). Because the sinking flux loss term, $F_{a l g} / z_{e u}$, only affects micro-phytoplankton, $\delta_{i}=1$ when $i=m$ and $\delta_{i}=0$ when $i=n$. The final term in Eq. 2.5 is the detrainment term, which represents dilution when the mixed layer is deepening such that $\mathrm{H}(\mathrm{x})=1$ if $\mathrm{x}>0$ and 0 otherwise (Siegel et al., 2014).

The estimated grazing rates on the nano- and micro- size fraction of NPP support the growth of small and large zooplankton respectively (Fig. 2-1). Large zooplankton also graze on small zooplankton. In this model, "large zooplankton" refers to those organisms which spend some portion or all of their diel cycle in the euphotic zone. It does not include zooplankton which are residents of the twilight zone and never cross the euphotic zone boundary. A portion of the total grazing flux into the large zooplankton box is converted to sinking fecal pellets, which includes both direct grazing on micro-phytoplankton and indirect grazing on nano-phytoplankton through grazing on small zooplankton. The fraction of large zooplankton fecal pellets that are produced in the euphotic zone and the twilight zone, respectively, will depend on the fraction of the large zooplankton community that is participating in DVM and the proportion of fecal pellets produced in the euphotic zone by migrating zooplankton. The fecal pellet flux out of euphotic zone $\left(F_{f e c}\right)$ is estimated as

$$
F_{f e c}=\left(p_{d v m} f_{f e c}+\left(1-p_{d v m}\right)\right)\left(m_{f e c} G_{m}+n_{f e c} G_{n}\right) z_{e u}
$$

and those fecal pellets not produced in the euphotic zone are carried by migrating zooplankton to the twilight zone. Fecal pellets produced in the twilight zone by migrators from the surface $\left(J_{f e c}\right)$ are modeled as

$$
J_{f e c}=p_{d v m}\left(1-f_{f e c}\right)\left(m_{f e c} G_{m}+n_{f e c} G_{n}\right) z_{e u}
$$

where $G_{m}$ and $G_{n}$ are the volumetric grazing rates on micro- and nano-phytoplankton respectively, $m_{f e c}$ and $n_{f e c}$ are the proportion of grazed carbon on each phytoplankton size class converted to large zooplankton fecal pellets, $p_{d v m}$ is the fraction of large zooplankton in the euphotic zone participating in DVM, and $f_{f e c}$ is the fraction of fecal pellets produced in the euphotic zone. The conversion of nano-phytoplankton biomass into large zooplankton fecal pellets is significantly less efficient due to the extra trophic step through small zooplankton, so we set $m_{f e c}$ equal to 0.3 and $n_{f e c}$ equal to 0.1 based on previous modeling studies (Michaels and Silver, 1988; Siegel et al., 2014). 
Observations of DVM biomass, based on the zooplankton biomass difference between day and night, indicate that the fraction of the zooplankton community participating in DVM can vary greatly and ranges from near zero to near one based on season and size fraction (Isla et al., 2015; Putzeys and Hernández-Leon, 2005; Takahashi et al., 2009). Analysis of acoustic scattering layer data by (Klevjer et al., 2016) found that an average of $50 \%$ of the mesopelagic backscatter participated in DVM, although this number varied significantly between different oceanographic regions and ranged from $20 \%$ to $90 \%$. We assumed a global value for $p_{d v m}$ of 0.5 based on these results.

The fraction of fecal pellets produced in the euphotic zone by migrating zooplankton depends on the time that migrating zooplankton spend in the euphotic zone relative to their gut clearance rate. The timing of DVM corresponds well to sunrise and sunset (Bianchi and Mislan, 2016), so we have assumed that the time that migrating zooplankton spend in the twilight zone is equal to day length. Based on evidence that grazing rates for migrating zooplankton are most intensive during the night (Haney, 1988), we assumed that all grazing by vertically migrating zooplankton occurs in the euphotic zone rather than the twilight zone. The only fecal pellets that are produced at depth are the result of one gut-full brought down from the surface. Therefore, the fraction of fecal pellets that migrating zooplankton produce in the twilight zone is equal to the ratio of one gut-full of grazed phytoplankton biomass to the total number of gut-fulls grazed over the daytime portion of the light cycle. Therefore, $f_{f e c}$ is defined as

$$
f_{f e c}=1-\frac{r}{24-\text { day length }}
$$

where $r$ is the mean gut clearance rate for grazing zooplankton and day length is represented as a function of latitude and day of the year following (Forsythe et al., 1995). There is considerable variability among measurements of zooplankton gut clearance rates resulting from differences in taxa, body size, life history stage, and temperature, with estimates ranging from 20 to 100 minutes (Atkinson et al., 1996; Bautista and Harris, 1992; Dam and Peterson, 1988). Clearance rates also tend to be slower for zooplankton participating in DVM (Atkinson et al., 1996). Here, we have chosen to use a constant value for gut clearance rate of 1 hour. Eq. 2.8 will fail to accurately represent the time fraction that migrating zooplankton spend in the euphotic zone when day length approaches either 0 or 24 hours, such as occurs near the poles, when DVM timing may not reflect the 


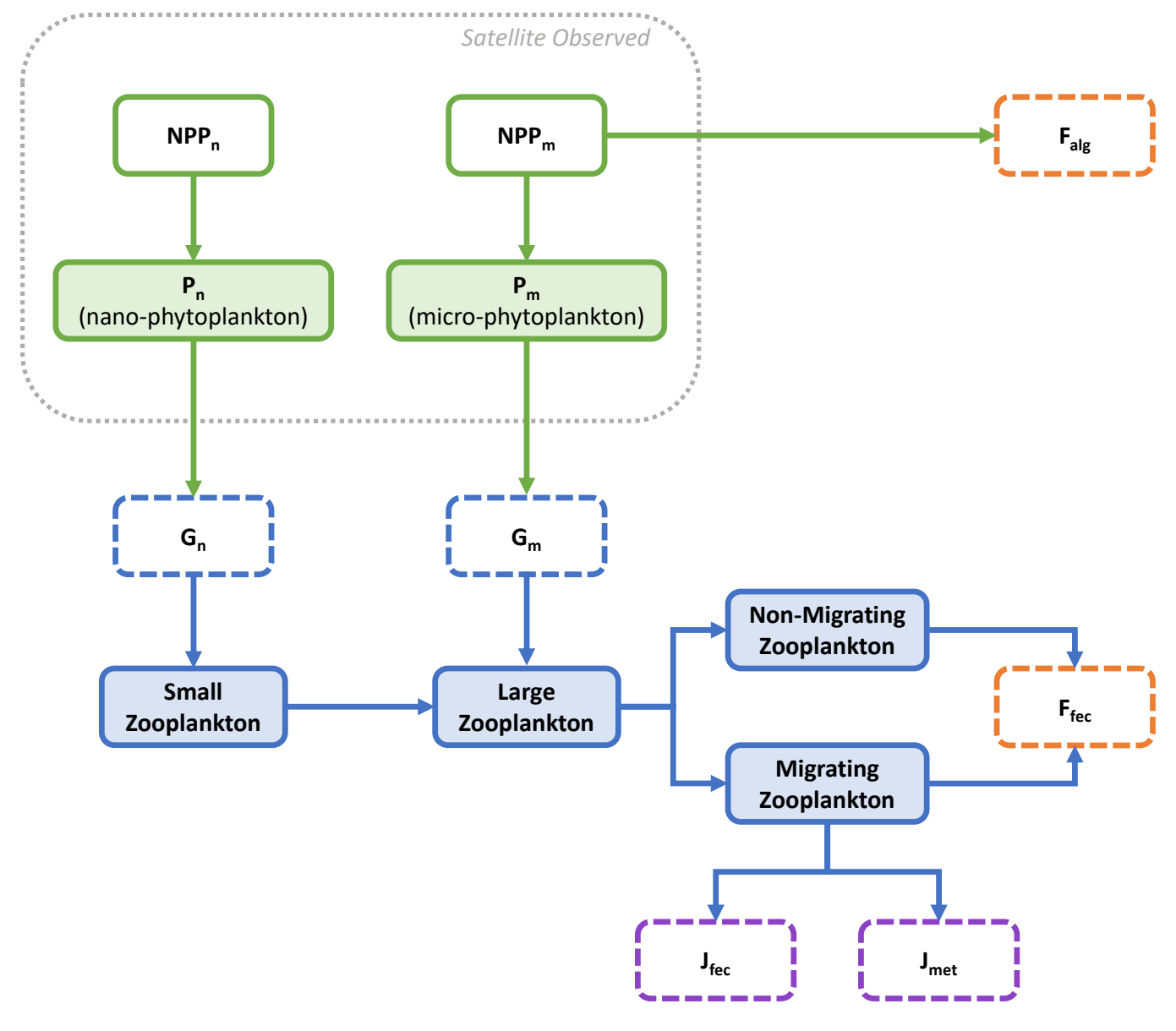

Figure 2-1: Conceptual diagram of the plankton food web as represented in the model and including the various export fluxes. Biomass standing stocks are indicated by solid lines and fluxes by dashed lines. Orange indicates a passive sinking export flux out of the euphotic zone and purple indicates DVM-mediated fluxes. The gray dashed line shows values that are empirical observations derived from satellite data products. 
length of the solar day (Fischer and Visbeck, 1993; Last et al., 2016). However, due to constraints on available satellite data as discussed below, the analysis of this model was restricted to the area between $60^{\circ} \mathrm{N}$ and $60^{\circ} \mathrm{S}$.

Grazed carbon which is not converted into fecal pellets is absorbed by zooplankton to be either metabolized or assimilated into biomass. It is important to note that we only modeled large zooplankton explicitly, and then only those large zooplankton which spend some part of their diel cycle in the euphotic zone, since small zooplankton were assumed to not participate in DVM (Isla et al., 2015). The absorbed carbon is the fraction of grazed phytoplankton biomass which is converted into large zooplankton biomass. Similar to the conversion of phytoplankton biomass to fecal pellets discussed above, the conversion of phytoplankton biomass to large zooplankton biomass is thought to be more efficient for the micro- size fraction than the nano- size fraction because of the additional trophic step through small zooplankton. We scaled the conversion of grazed nano-phytoplankton into large zooplankton by the ratio of $n_{f e c}: m_{f e c}$ to reflect the loss of organic carbon from small zooplankton respiration prior to consumption by large zooplankton. Grazed carbon absorbed by large zooplankton $\left(G_{a b s}\right)$ is calculated as

$$
G_{a b s}=\left(1-m_{f e c}\right) G_{m}+\frac{n_{f e c}}{m_{f e c}}\left(1-m_{f e c}\right) G_{n}
$$

A fixed proportion of the carbon absorbed by large zooplankton is respired and the relative proportion of this respiration which occurs in the twilight zone is based on the temperature difference between the surface and a given depth. $J_{m e t}$, the production of respiratory DIC in the twilight zone by vertically migrating zooplankton, is given by

$$
J_{m e t}=p_{m e t} p_{d v m}\left(f_{m e t} G_{a b s} z_{e u}\right)
$$

where

$$
p_{m e t}=\frac{Q_{10}^{\frac{T(z)-T(0)}{10}}}{Q_{10^{\frac{T(z)-T(0)}{10}}+1}^{10}}
$$

$f_{m e t}$ is the fraction of absorbed carbon that is metabolized, $p_{m e t}$ is the fraction of total metabolism that occurs in the twilight zone, $Q_{10}$ is the metabolic temperature coefficient, $\mathrm{T}(\mathrm{z})$ is the temperature at depth $\mathrm{z}$, and $\mathrm{T}(0)$ is the temperature at the surface. We set $f_{m e t}$ equal to 0.5 and $Q_{10}$ equal to 
2 (Ikeda, 2014; Steinberg and Landry, 2017).

Large zooplankton biomass in the euphotic zone $(\mathrm{Z})$ was modeled as

$$
\frac{d Z}{d t}=\left(1-f_{m e t}\right) G_{a b s}-m_{z}\left(Z-Z_{0}\right)-p_{z}\left(Z-Z_{0}\right)^{2}
$$

where $m_{z}$ and $p_{z}$ are linear and quadratic mortality rates, respectively, and $Z_{0}$ is a lower threshold for zooplankton biomass, which was included to stabilize the model. We chose values of $0.05 \mathrm{~d}^{-1}$ for

$m_{z}$ and $0.15 \mathrm{mgC}^{-1} \mathrm{~m}^{3} \mathrm{~d}^{-1}$ for $p_{z}$ to be consistent with previous models of surface food webs (Doney et al., 1996; Fasham et al., 1990). This equation is implemented with $\mathrm{Z}(\mathrm{t})$ as a state variable that changes dynamically in time over a climatological seasonal cycle at each grid location.

\section{The Twilight Zone Module}

We assumed that migrating zooplankton in the twilight zone were normally distributed about a mean migration depth located at the $10^{-3} \mathrm{~W} / \mathrm{m}^{2}$ isolume (Bianchi et al., 2013b). We parameterized the variability in zooplankton DVM depth with a truncated Gaussian function that was $100 \mathrm{~m}$ wide and had a half power thickness of $50 \mathrm{~m}$ based on acoustic measurements of the thickness of the migrating zooplankton layer in DVM events by (Bianchi and Mislan, 2016). The depth of this isolume is calculated using satellite measurements of surface photosynthetically active radiation (PAR) and two separate light attenuation coefficients $(k)$, one for the euphotic zone and one for the twilight zone. The PAR attenuation coefficient in the euphotic zone was calculated from satellite-observed euphotic depth, where the variable $z_{e u}$ is defined as the depth at which PAR is $1 \%$ of the measured surface value. The value of $k$ for the twilight zone was set to 0.03 based on minimum in situ light attenuation measurements (Son and Wang, 2015). The depth of migration was further restricted by in situ oxygen concentration. We defined the lower limit of zooplankton hypoxia tolerance as 15 $\mu \mathrm{mol} / \mathrm{L}$. The mean depth of migration is then the shallower depth of the $10^{-3} \mathrm{~W} / \mathrm{m}^{2}$ isolume and the $15 \mu \mathrm{mol} / \mathrm{L}$ oxygen isopleth.

Large zooplankton fecal pellets produced in the twilight zone $\left(J_{f e c}\right)$ contribute to the sinking POC flux composed of algal aggregates and zooplankton fecal pellets produced in the euphotic zone. We assumed that the gut clearance rate and the migration transit time were roughly equivalent based on global estimates of the mean transit time (1.75 hours) from acoustic data (Bianchi and 
Mislan, 2016). Fecal pellets carried by vertical migrators were distributed along the migration path using a uniform distribution between the euphotic depth and the maximum migration depth to produce a depth-dependent function of fecal pellet production in the twilight zone $\left(J_{f e c}(z)\right)$. In contrast, we assume that the migration transit time is small compared to the time spent at depth such that DIC produced by migrating zooplankton in the twilight zone is centered around the maximum migration depth rather than distributed along the migration path. We calculated the depth-dependent distribution of the production of metabolic DIC around the maximum migration depth $\left(J_{\text {met }}(z)\right)$ by taking the convolution of $J_{\text {met }}$ with the truncated Gaussian described above that represents variability in the concentration of migrating zooplankton around the target depth.

The shape of the POC flux profile in the euphotic zone results from the combined influence of physical and biological factors including the breakage of large particles into smaller slower-sinking ones, remineralization by the attached microbial community, and grazing by mesopelagic (nonmigrating) zooplankton and fish, all of which serve to attenuate the flux over depth, and the influx of fecal pellets produced by migrating large zooplankton, which adds to the flux. We parameterized the physical and biological attenuation of the POC flux as the exponential decay of the labile portion of the flux and included the effect of temperature and dissolved oxygen concentration on the remineralization rate (Laufkötter et al., 2017; Lima et al., 2014). The POC flux profile in the twilight zone $(F(z))$ was discretized as a function of depth such that

$$
F\left(z_{i}\right)=(1-\alpha) F\left(z_{i-1}\right)+\alpha F\left(z_{i-1}\right) \exp \left[\frac{-1}{\lambda} e^{k_{t} T\left(z_{i}\right)} \frac{O_{2}\left(z_{i}\right)}{O_{2}\left(z_{i}\right)+k_{o}} \Delta z\right]+J_{f e c}\left(z_{i}\right)
$$

with upper boundary condition $F\left(z_{e u}\right)=F_{e u}$. Algorithmically, this means that at each depth bin we apply the exponential decay function to the influx of sinking particles from the depth bin above plus the input of fecal pellets from $J_{f e c}(z) . \Delta z$ is the depth bin width, $\alpha$ is the labile fraction of the flux, $\lambda$ is the characteristic length scale of remineralization, $k_{t}$ is the temperature dependence parameter, and $k_{o}$ is the oxygen half-saturation coefficient. The characteristic length scale is defined as the ratio of sinking speed $(\mathrm{m} / \mathrm{d})$ to remineralization rate $(1 / \mathrm{d})$. Within a temperature dependent parameterization, $\lambda$ is the characteristic length scale at $0^{\circ} \mathrm{C}$ where the temperature coefficient is 1. While the reference $\lambda$ remains constant, the remineralization length scale is modulated by the temperature dependence term such that the functional length scale is equal to $200 \mathrm{~m}$ at $0{ }^{\circ} \mathrm{C}$ and 
declines to $200 / \mathrm{e} \mathrm{m}$ at $20^{\circ} \mathrm{C}$. The parameter values for this function (Table 2.2) are consistent with previous models of mesopelagic remineralization of the POC flux (Laufkötter et al., 2017; Lima et al., 2014). We used 2009 World Ocean Atlas data (annual climatology, $1^{\circ}$ grid) to define the mesopelagic temperature $\left(\mathrm{T}(\mathrm{z}),{ }^{\circ} \mathrm{C}\right)$ and oxygen concentrations $\left(\mathrm{O}_{2}, \mu \mathrm{mol} / \mathrm{L}\right)$.

Oxygen consumption and the production of metabolic DIC in the model is the sum of remineralization of the vertical POC flux and the metabolism of vertically migrating zooplankton. The respiratory source term $(\mathrm{R}(\mathrm{z}))$, or equivalently the transformation of organic carbon in the euphotic zone to DIC in the twilight zone, is defined as

$$
R(z)=\frac{d D I C}{d t}=-\frac{d F}{d z}+J_{m e t}(z)
$$

We defined three metrics to quantify the relative contributions of model variables to water column biogeochemistry. The DVM export ratio is the ratio of the DVM-mediated export flux to the total export flux to below the euphotic zone,

$$
\text { DVM Export Ratio }=\frac{J_{d v m}}{J_{d v m}+F_{e u}}
$$

Similarly, the DVM respiration ratio is the ratio of the respiration performed by vertically migrating zooplankton to the integrated respiration in the mesopelagic from the euphotic depth to $1000 \mathrm{~m}$.

$$
\text { DVM Respiration Ratio }=\frac{J_{m e t}}{\int_{z_{e u}}^{1000} R(z) d z}
$$

Finally, we defined the weighted depth of respiration to quantify how DVM activity by zooplankton pushes the production of DIC and oxygen utilization deeper into the water column.

$$
\text { Weighted Depth of Respiration }=\frac{\int_{z_{e u}}^{1000} z * R(z) d z}{\int_{z_{e u}}^{1000} R(z) d z}
$$

Because the weighted depth of respiration depends on both DVM activity and variability in the temperature and oxygen concentrations, we report the "Respiration Depression" as the difference between the weighted depth of respiration from the DVM model and a baseline remineralization profile that includes only the respiration of sinking particles and no DVM $\left(p_{d v m}=0\right)$. 
Table 2.2: DVM model parameters

\begin{tabular}{||c|c|c|c||}
\hline Parameter & Description & Value & Units \\
\hline$f_{a l g}$ & fraction of $N P P_{m}$ exported as aggregates & 0.1 & \\
$m_{p}$ & phytoplankton linear mortality & 0.1 & $\mathrm{~d}^{-1}$ \\
$p_{d v m}$ & fraction of zooplankton vertically migrating & 0.5 & \\
$m_{f e c}$ & fraction of grazed $N P P_{m}$ converted to fecal pellets & 0.3 & \\
$n_{f e c}$ & fraction of grazed $N P P_{n}$ converted to fecal pellets & 0.06 & \\
$f_{f e c}$ & fraction of fecal pellet production in the euphotic zone & var. & \\
$r$ & gut clearance rate & 1 & $\mathrm{hr}$ \\
$p_{m e t}$ & fraction of metabolism in the twilight zone & var. & \\
$Q_{10}$ & $Q_{10}$ temperature coefficient & 2 & \\
$f_{m e t}$ & fraction of absorbed carbon metabolized & 0.5 & \\
$m_{z}$ & zooplankton linear mortality & 0.05 & $\mathrm{~d}^{-1}$ \\
$p_{z}$ & zooplankton quadratic mortality & 0.01 & $\mathrm{mgC}^{-1} \mathrm{~m}^{3} \mathrm{~d}^{-1}$ \\
$k$ & PAR attenuation coefficient & 0.03 & $\mathrm{~m}^{-1}$ \\
$\alpha$ & biomass labile fraction & 0.8 & \\
$\lambda$ & remineralization length scale & 200 & $\mathrm{~m}^{-1}$ \\
$k_{t}$ & remineralization temperature dependence & 0.05 & ${ }^{\circ} \mathrm{C}^{-1}$ \\
$k_{o}$ & oxygen half-saturation coefficient & 10 & $\mu \mathrm{mol}^{\mathrm{L}}$ \\
$\Delta z$ & depth interval & 10 & $\mathrm{~m}$ \\
\hline
\end{tabular}


Together these metrics quantify the magnitude of the effect that zooplankton DVM has on carbon export out of the euphotic zone and on DIC production and oxygen utilization in the twilight zone. Higher values of the DVM export ratio and the DVM respiration ratio indicate that zooplankton migrations are accounting for greater fractions of carbon export and oxygen utilization in the twilight zone. A positive value of the respiration depression indicates that zooplankton DVM is pushing the DIC production and oxygen utilization deeper in the water column.

\subsubsection{Application of the Model}

We first applied the above 1-dimensional model to monthly mean satellite observations from the Bermuda Atlantic Time Series (BATS) scientific station at $31^{\circ} 40^{\prime} N, 64^{\circ} 10^{\prime} W$. We calculated the depth profile of the vertical POC flux and the profile of DIC production in the twilight zone, and the export ratio over the seasonal cycle (total carbon export out of the euphotic zone divided by depth-integrated NPP). For this test case, and in contrast to the global model discussed below, the mean and standard deviation (SD) calculated for this grid point represents seasonal variability over monthly means from the annual climatology and does not account for regional variability.

Next, we applied the 1-dimensional model to a global, $1^{\circ}$ grid. We kept the parameters of the model constant across the global domain. These parameter estimates represent mean or characteristic values that describe a variety of different phytoplankton and zooplankton communities which occur in the world's oceans. Differences between grid points are the result of variability in the drivers of the model - observed NPP, phytoplankton biomass, size structure, temperature, and oxygen concentration. A baseline simulation was run with $p_{d v m}=0$ for a control scenario in which there was no DVM. Global patterns were based on the annual mean over the seasonal cycle at each grid point. The mean and standard deviation calculated for the global model includes both seasonal and regional variability. Only grid points that had at least eight months of available satellite data over the seasonal cycle were included in the analysis. This excluded most of the grid at high latitudes due to the persistence of clouds and sea ice cover, and the extended duration of the polar night.

We tested the sensitivity of the model to each of the parameters numerically by increasing each of the parameters by $1 \%$ and calculating the resulting change in a suite of model outputs that serve to characterize the magnitude and composition of the global export flux: the total global export flux, the average export ratio, the average magnitude of the passive sinking flux, the average 
magnitude of the DVM-mediated export flux, the DVM export ratio, the DVM respiration ratio, and the respiration depression. The choice of a $1 \%$ perturbation is not intended to reflect realistic estimates of parameter error, but instead is used as a baseline to test the relative sensitivity of the model to each parameter. A response close to or greater than 1\% (in either the positive or negative direction) indicates a relatively high sensitivity to that parameter. The assumption implicit to this approach is that parameter error falls within a region of linear stability. We tested the model response to parameter perturbations of up to $100 \%$ and confirmed that the sensitivity is linear for most model parameters and outputs, indicating the sensitivity results presented here should scale well with parameter errors larger than $1 \%$.

\subsection{Results}

\subsubsection{1-D Model at BATS}

Annual mean $( \pm \mathrm{SD}) \mathrm{NPP}$ at the BATS grid point was $277 \pm 58 \mathrm{mgC} / \mathrm{m}^{2} \mathrm{~d}$. Satellite climatologies of phytoplankton biomass show a seasonal mixed layer phytoplankton bloom dominated by nano-phytoplankton with peaks in March and September (Fig. 2-2). Micro-phytoplankton biomass was low over the entire seasonal cycle, with a small increase in February, just before the nanophytoplankton bloom. During the summer, when nano-phytoplankton biomass was high, microphytoplankton biomass was near zero. Modeled large zooplankton biomass was lower than phytoplankton biomass and showed significantly less seasonality. A small peak in zooplankton occurred during March and April following the increase in micro-phytoplankton. The mean $( \pm \mathrm{SD})$ nanophytoplankton biomass was $10 \pm 2.7 \mathrm{mgC} / \mathrm{m}^{3}$, mean micro-phytoplankton biomass was $0.8 \pm 0.79$ $\mathrm{mgC} / \mathrm{m}^{3}$, and mean zooplankton biomass was $3.5 \pm 0.7 \mathrm{mgC} / \mathrm{m}^{3}$. Mean euphotic depth at BATS was $82 \pm 11 \mathrm{~m}$, mean mixed layer depth was $52 \pm 33 \mathrm{~m}$, and mean depth of the $10^{-3} \mathrm{~W} / \mathrm{m}^{2}$ isolume was $378 \pm 27$ m (Fig. 2-2).

Fig. 2-3 shows the monthly averages of the magnitude of the carbon export fluxes $F_{e u}$ and $J_{d v m}$ at BATS, as well as the quantitative metrics used to evaluate the relative magnitude of these fluxes. Export from the euphotic zone by passive sinking $\left(F_{e u}\right)$ showed significant seasonality with a peak in March and April corresponding to the yearly maximum in micro-phytoplankton and zooplankton biomass. $F_{e u}$ was dominated by the sinking fecal pellet flux. $J_{d v m}$ is approximately one-third as 

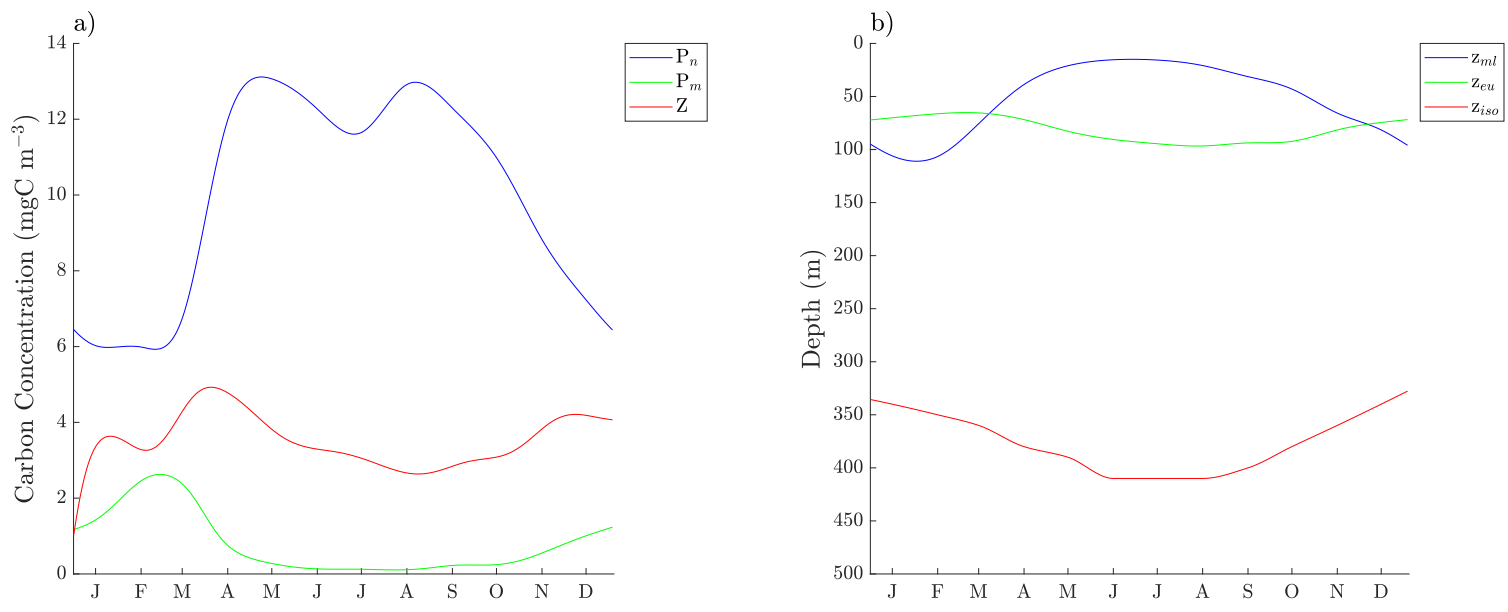

Figure 2-2: (a) Biomass of nano- and micro-phytoplankton $\left(\mathrm{P}_{n}, \mathrm{P}_{m}\right)$ and zooplankton (Z) in the mixed layer over the seasonal cycle at the Bermuda Atlantic Time Series grid point. (b) Depth of the euphotic zone $\left(\mathrm{z}_{e u}\right)$, the mixed layer $\left(\mathrm{z}_{m l}\right)$, and the $10^{-3} \mathrm{~W} / \mathrm{m}^{2}$ isolume $\left(\mathrm{z}_{\text {iso }}\right)$. 
large as $F_{e u}$, and in contrast is dominated by metabolic production of DIC in the twilight zone rather than fecal pellets. Model estimates of the export ratio, DVM export ratio, and the DVM respiration ratio showed very little seasonality. The mean $( \pm \mathrm{SD})$ export ratio at BATS was $0.07 \pm 0.03$, with an annual mean fraction of 0.05 of the NPP exported by $F_{\text {eu }}$ and 0.02 by $J_{d v m}$. The annual mean DVM export ratio was $0.23 \pm 0.02$ and the annual mean DVM respiration ratio was $0.21 \pm 0.02$, indicating that zooplankton DVM activity accounted for approximately equal proportions of total export and total twilight zone respiration. The annual mean respiration depression, compared to the model run which did not account for DVM, was $54 \pm 8 \mathrm{~m}$. Respiration depression showed significant seasonality with much larger values in the summer than in the winter.

Fig. 2-4 shows the depth dependence of the vertical POC flux and the production of DIC, or equivalently, oxygen utilization in the twilight zone at BATS. While DVM activity is responsible for a large local maximum in DIC production at depth, it has relatively little impact on the vertical profile of sinking POC because the magnitude of $J_{f e c}$ is much smaller than $J_{m e t}$ and because fecal pellets are deposited uniformly along the migration path and DIC is all produced at the maximum depth. In the absence of any DVM behaviors, the profiles of both POC flux and DIC production would be monotonically decreasing (Fig. 2-4).

\subsubsection{Global Model Dynamics}

Annual mean $( \pm \mathrm{SD})$ global NPP was $414 \pm 194 \mathrm{mgC} / \mathrm{m}^{2} \mathrm{~d}$, with an integrated global carbon flux of $6.5 \mathrm{PgC} / \mathrm{yr}$. The global mean export ratio was $0.12 \pm 0.05$. Passive sinking of particles out of the euphotic zone $\left(F_{\text {eu }}\right)$ accounted for an annual mean $( \pm \mathrm{SD})$ fraction of $0.1 \pm 0.05$ of the global NPP and the DVM-mediated flux $\left(J_{d v m}\right)$ accounted for an additional $0.02 \pm 0.01$ of global NPP. The carbon transported by zooplankton DVM was a significant contributor to the export flux. In the control run with no DVM, the global carbon export flux was 5.7 $\mathrm{PgC} / \mathrm{yr}$ and the annual mean export ratio was $0.10 \pm 0.04$. The integrated global flux values represent an area of the ocean that is $3.16 \times 10^{8} \mathrm{~km}^{2}$, which is approximately $20 \%$ smaller than the total area of the ocean (Siegel et al., 2014). Due to constraints on available data for grid points found at high latitudes, polar blooms are largely absent from these calculations.

The global distributions of model drivers and state variables are shown in Fig. 2-5. NPP is higher near the coast and in upwelling zones, including eastern boundaries and around the equator. The 

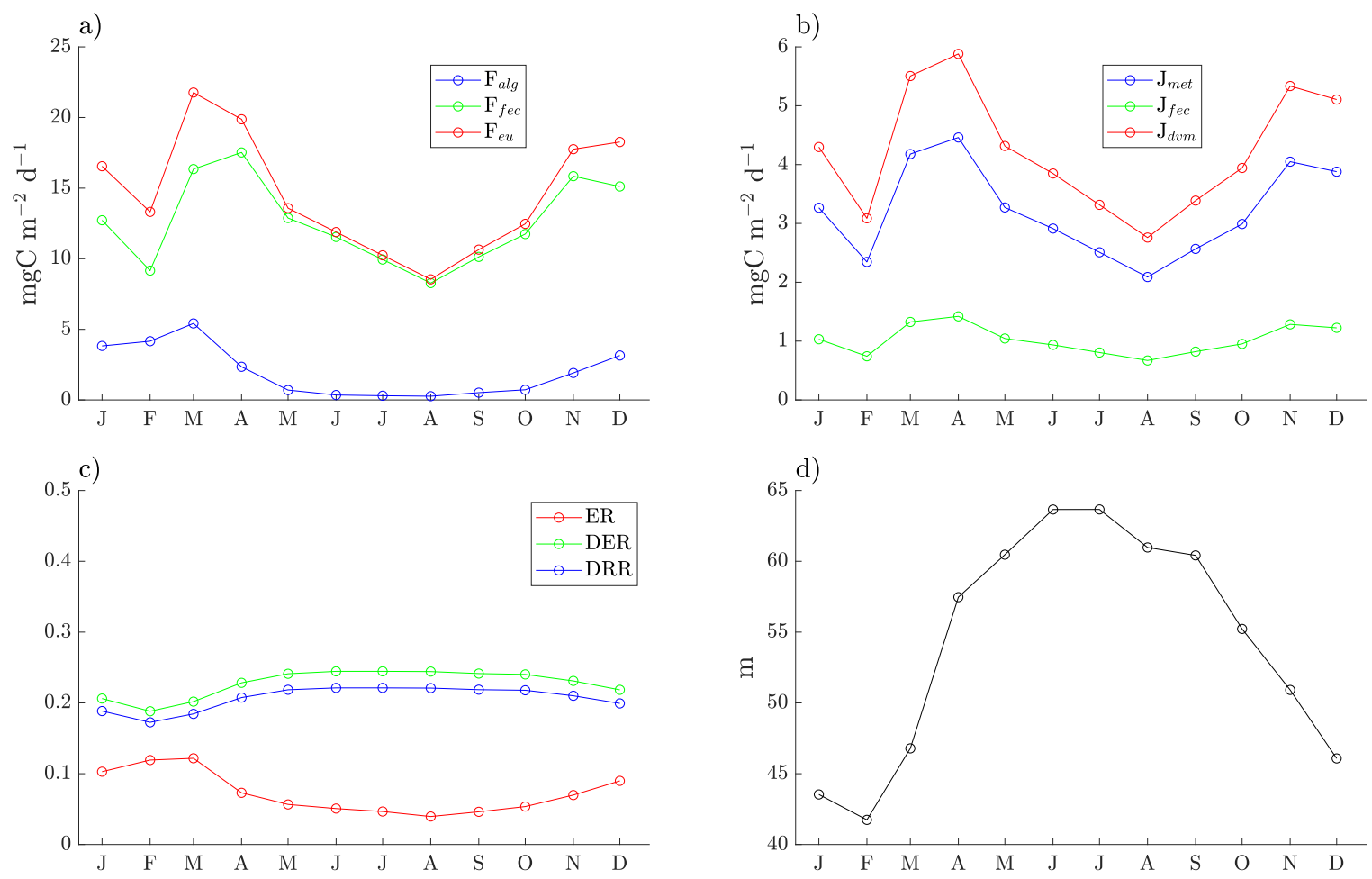

Figure 2-3: Monthly means of (a) the passive sinking flux $\left(F_{\text {eu }}\right)$, (b) the DVM-mediated export flux $\left(J_{d v m}\right),(\mathrm{c})$ the export ratio (ER), DVM export ratio (DER), and the DVM respiration ratio (DRR) and $(\mathrm{d})$ respiration depression (RD) at BATS. Note the difference of vertical scales between (a) and (b). 

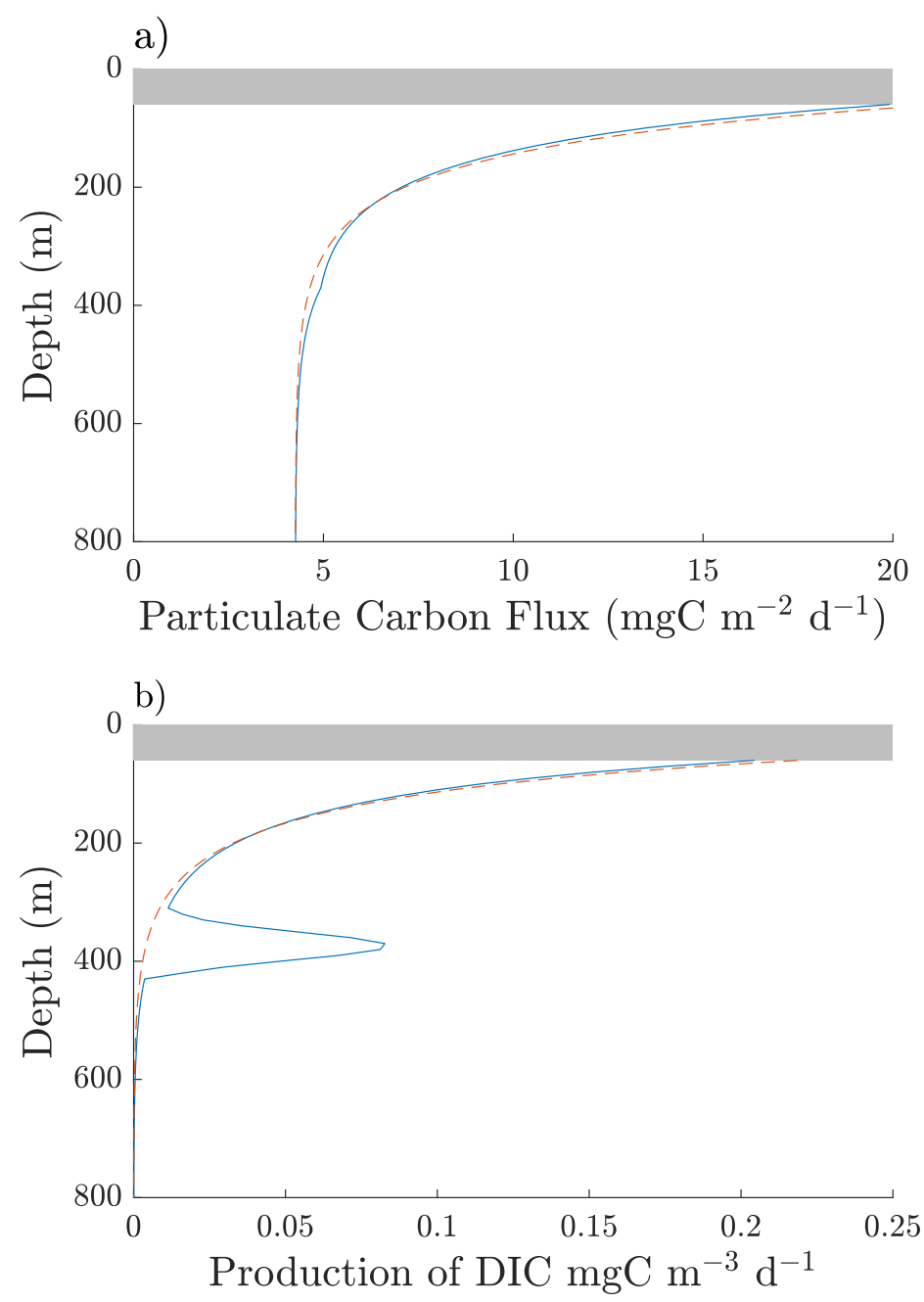

Figure 2-4: Vertical profiles at BATS of (a) particulate carbon flux $(\mathrm{F}(\mathrm{z})$ ) and (b) the production of DIC $(\mathrm{R}(\mathrm{z}))$ in the twilight zone for the month of April, during the peak of the phytoplankton bloom. The euphotic zone is indicated by the gray box. The blue line represents a model run when $p_{d v m}=0.5$ and the red dashed line represents the control run when $p_{d v m}=0$. 
high NPP areas tend to be dominated by micro-phytoplankton, while nano-phytoplankton dominate the ecosystem in the center of ocean gyres in the subtropical latitudes. Modeled large zooplankton biomass had similar patterns to NPP. The global mean ( \pm SD) zooplankton biomass was $2.8 \pm 0.9$ $\mathrm{mgC} / \mathrm{m}^{3}$. The mean DVM depth was $356 \pm 41 \mathrm{~m}$. Migration depth was typically deeper in the subtropics where incident light is high and chlorophyll concentrations are low, although mesopelagic oxygen concentration had a strong shoaling effect. Large hypoxic regions, such as the north and south subtropical eastern Pacific and the northern Indian ocean had migration depths significantly lower than the global mean. The modeled migration depths were only slightly shallower, on average, than the global mean DVM depth reported by (Bianchi and Mislan, 2016), who estimated a mean value of $411 \mathrm{~m}$ based on acoustic doppler current profiler data from 1990 to 2010. This discrepancy may indicate that modeling migration depth by assuming a constant target isolume modified by low oxygen concentrations may be insufficient.

The magnitude of the total export flux, as well as each of the component fluxes $F_{\text {eu }}$ and $J_{d v m}$, was larger in highly productive coastal zones and upwelling regions (Fig. 2-6). However, despite the magnitude of these fluxes following patterns in NPP, the relative contribution of $F_{e u}$ and $J_{d v m}$ to total export displayed distinctly different global patterns (Fig. 2-7). Both the DVM export ratio and the DVM respiration ratio, which define the proportional contributions of DVM activity to total export and mesopelagic respiration, were higher in the subtropical latitudes where the community was dominated by nano-phytoplankton (Fig. 2-5). Respiration depression was highest in the subtropical latitudes as well, with significantly reduced values in hypoxic regions where the migration depth was very shallow (Fig. 2-7). The global mean ( \pm SD) DVM export ratio was $0.16 \pm 0.04$. The global mean DVM respiration ratio was $0.16 \pm 0.06$. The global mean respiration depression was $30 \pm 18 \mathrm{~m}$. A summary of the global results is presented in Table 2.3.

\subsubsection{Model Sensitivity}

We tested sensitivity by calculating the model response to a $1 \%$ change in each of the parameters. Proportional responses close to or greater than $1 \%$ indicate a relatively high sensitivity to a given parameter. All of the model responses fell between $-2 \%$ and $+1 \%$. The modeled total global export flux was more robust to changes in the parameter values than the relative contributions of $F_{e u}$ and $J_{d v m}$ to total export (Fig. 2-8). The global export flux was most sensitive to $n_{f e c}$, which controls 


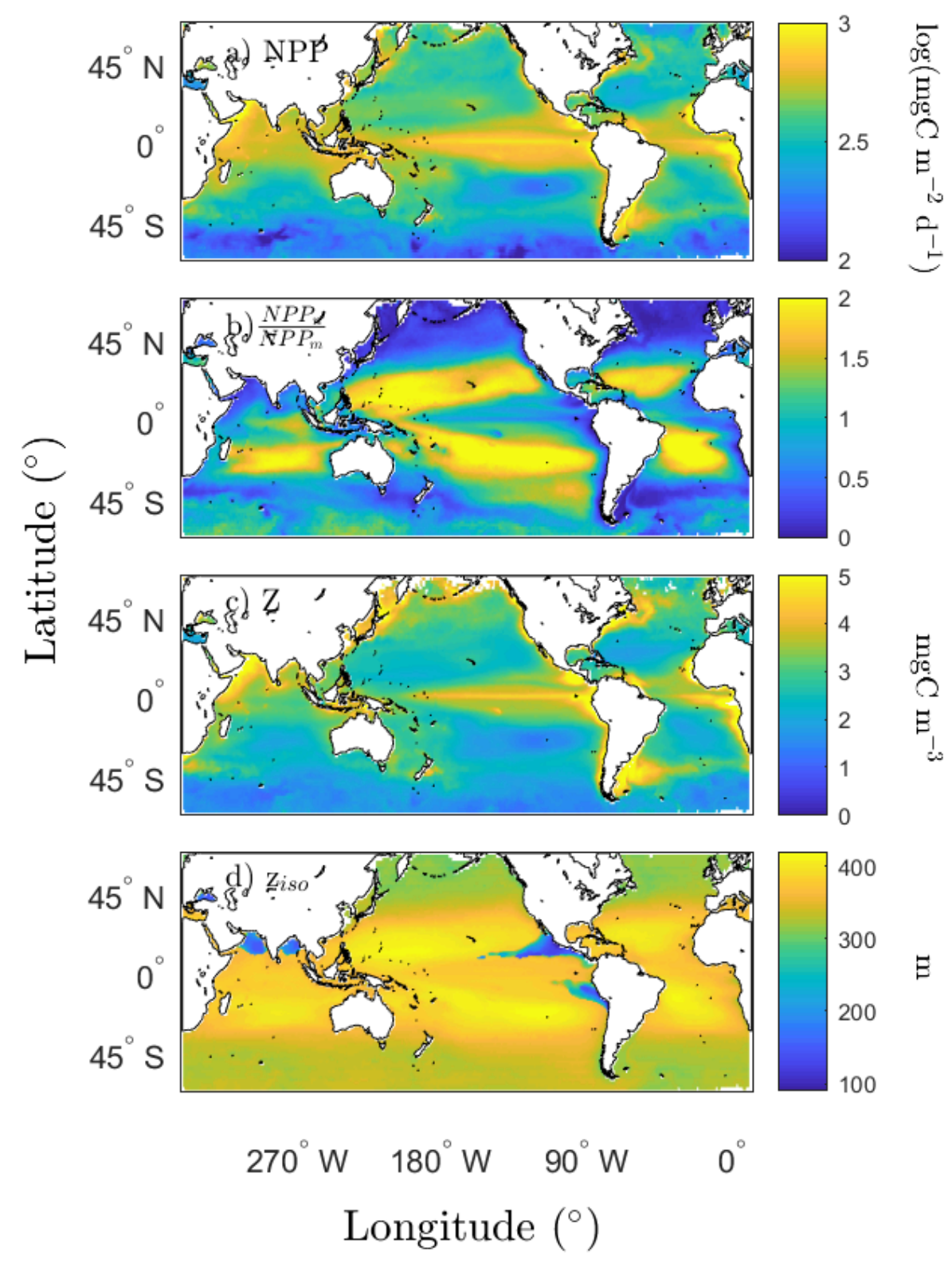

Figure 2-5: Global distribution of (a) net primary productivity (NPP), (b) the NPP biovolume ratio $\left(\mathrm{NPP}_{n} / \mathrm{NPP}_{m}\right)$, (c) zooplankton biomass $(\mathrm{Z})$, and $(\mathrm{d})$ migration depth $\left(\mathrm{z}_{i s o}\right)$. The value at each grid point is the annual mean over the seasonal cycle. 
Table 2.3: Summary of global statistics. Annual mean and standard deviation values include temporal variability across months in the yearly climatology and spatial variability across the global model domain.

\begin{tabular}{||c|c|c||}
\hline Statistic & \multicolumn{2}{|c||}{ Mean (SD) } \\
& $p_{d v m}=0$ & $p_{d v m}=0.5$ \\
\hline NPP $\left(\mathrm{mgC} \mathrm{m}^{-2} \mathrm{~d}^{-1}\right)$ & $414(194)$ & $414(194)$ \\
Global Export Flux $(\mathrm{PgC} / \mathrm{yr})$ & 5.7 & 6.5 \\
Export Ratio & $0.10(0.04)$ & $0.12(0.05)$ \\
DVM Export Ratio & & $0.16(0.04)$ \\
DVM Respiration Ratio & & $0.16(0.06)$ \\
Respiration Depression $(\mathrm{m})$ & & $30(18)$ \\
\hline
\end{tabular}



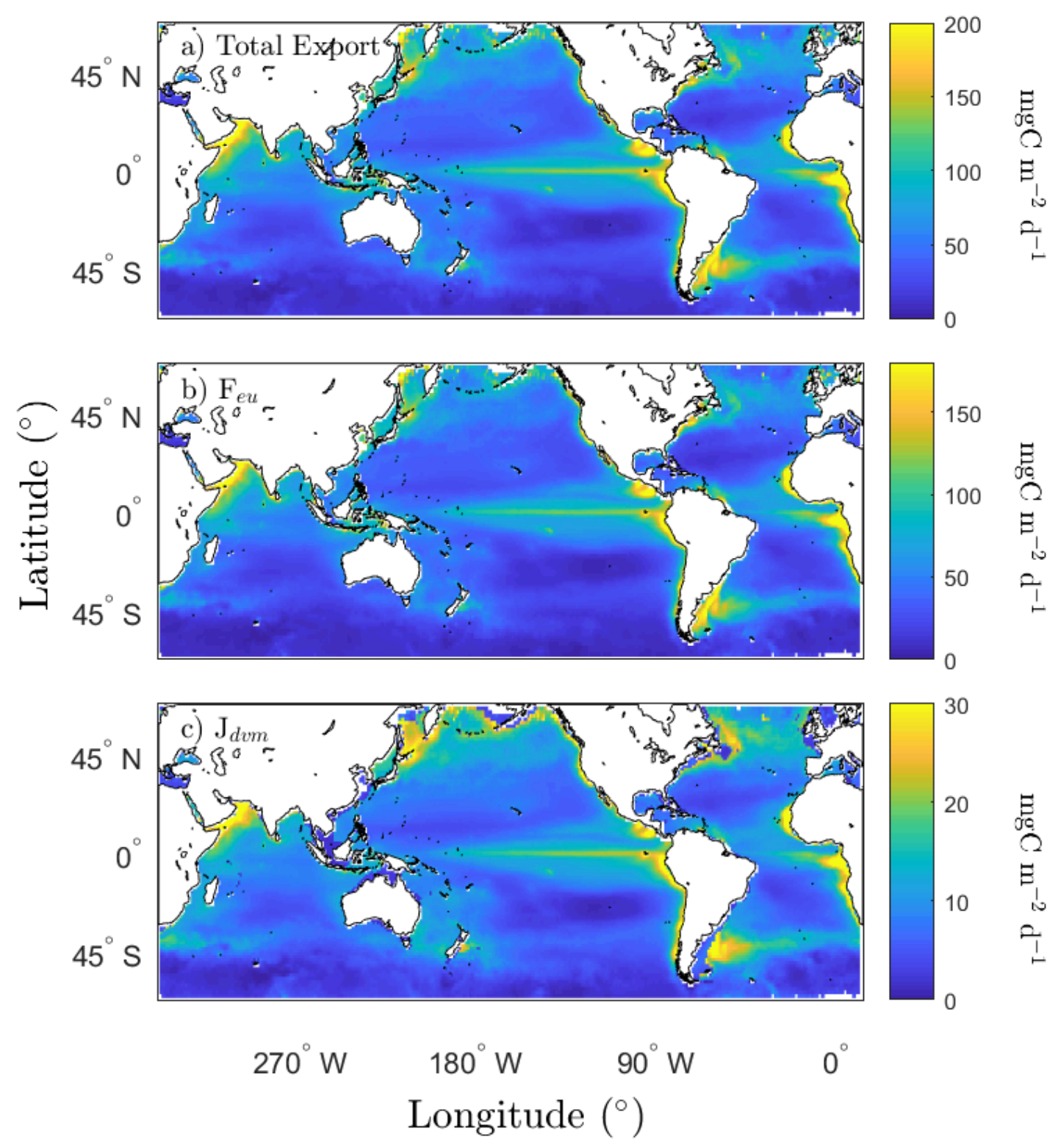

Figure 2-6: Global distribution of the magnitude of the (a) total export flux, (b) the passive sinking export flux $\left(\mathrm{F}_{e u}\right)$, and (c) the DVM-mediated export flux $\left(\mathrm{J}_{d v m}\right)$. The value at each grid point is the annual mean over the seasonal cycle. 


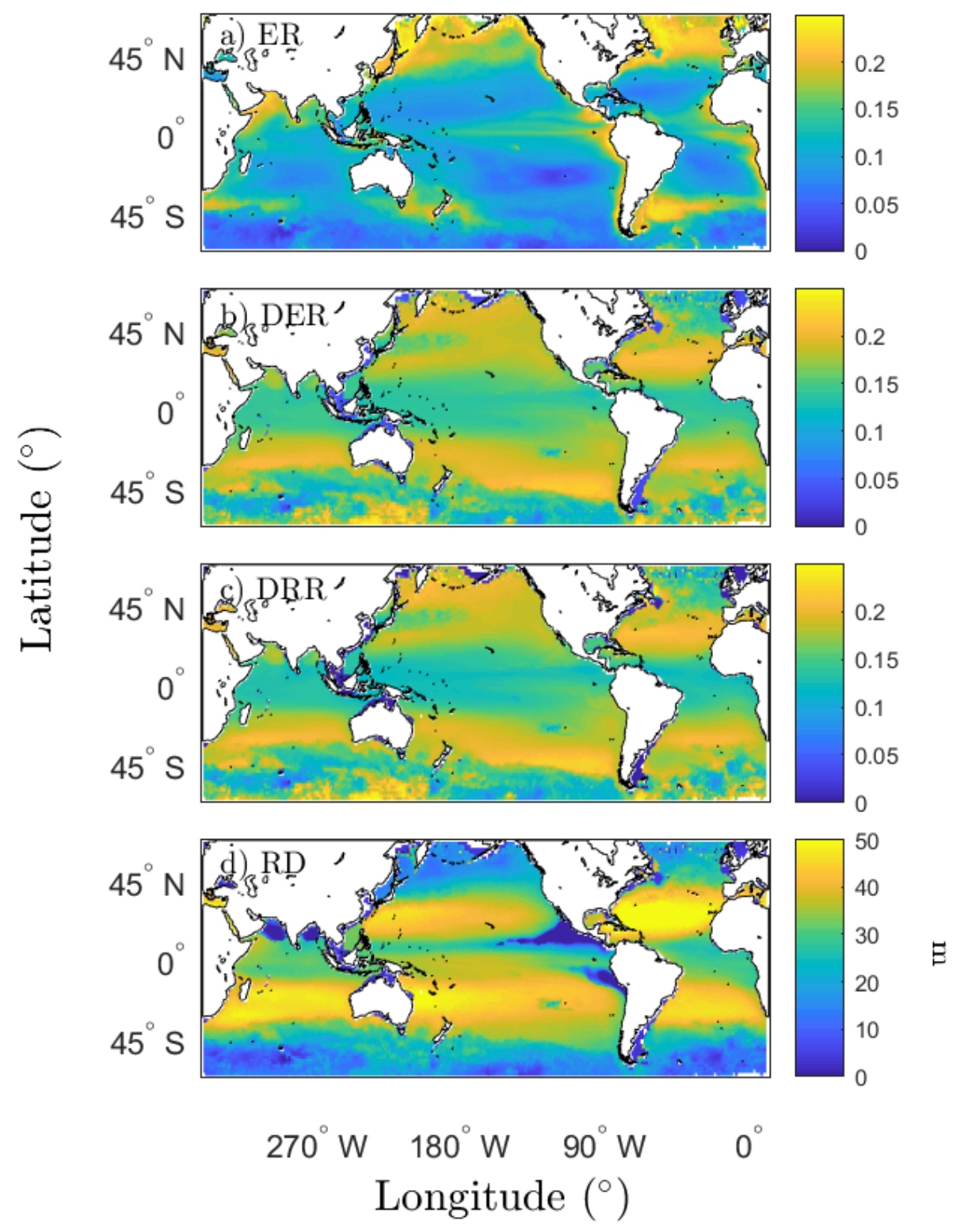

Figure 2-7: Global distribution of the (a) export ratio (ER), (b) DVM export ratio (DER), (c) DVM respiration ratio $(\mathrm{DRR})$, and $(\mathrm{d})$ respiration depression $(\mathrm{RD})$. The value at each grid point is the annual mean over the seasonal cycle. 
the fraction of grazed phytoplankton biomass in the nano size class that is converted into large zooplankton fecal pellets. Importantly, the relative value of $n_{f e c}$ to $m_{f e c}$ also parameterizes the trophic efficiency of large zooplankton grazing on nano-phytoplankton indirectly through the consumption of small zooplankton, and so $n_{f e c}$ also plays a role in determining how much total phytoplankton biomass is absorbed by large zooplankton and, ultimately, how much carbon is respired by vertically migrating zooplankton. The relative contributions of $F_{e u}$ and $J_{d v m}$ to total export - quantified by the DVM export ratio, the DVM respiration ratio, and the respiration depression - were all most sensitive to $f_{f e c}, p_{d v m}$, and $f_{m e t}$. These three parameters do not determine the total amount of carbon exported, but contribute heavily to determining the partitioning of exported carbon between passive sinking and active transport pathways.

\subsection{Discussion}

We approached the challenge of modeling diel vertical migration by assuming general principles and parameters for the pelagic ecosystem that were applied globally. Neither model formulation nor parameter values were changed across the model domain. Instead, variability in the inputs (NPP, phytoplankton biomass and size structure) were the drivers of the system rather than regional differences in ecosystem description. This conceptual approach allowed us to work around the problem of incomplete knowledge concerning regional differences in the plankton community and provides a blueprint for future work. Model estimates of the global carbon export flux (6.5 PgC/yr) are consistent with recent model estimates of the global surface POC flux ranging from 5-12 PgC/yr (Siegel et al., 2016). The flux of carbon to below the euphotic zone mediated by zooplankton DVM is an important part of the biological pump, increasing the global export flux by $14 \%$.

The one-dimensional model run at the BATS grid point provides a case study which can be used to evaluate the performance of the model in the subtropics, a region where the effect of DVM activity on carbon export and mesopelagic biogeochemistry is significant. Overall, the model does a reasonable job of representing the biological pump at BATS considering the model was designed to represent a generalized global ecosystem and was not specifically parameterized for the BATS location. Satellite climatologies of production and phytoplankton biomass are consistent with long-term in situ measurements at the BATS station (Steinberg et al., 2001). Both satellite 


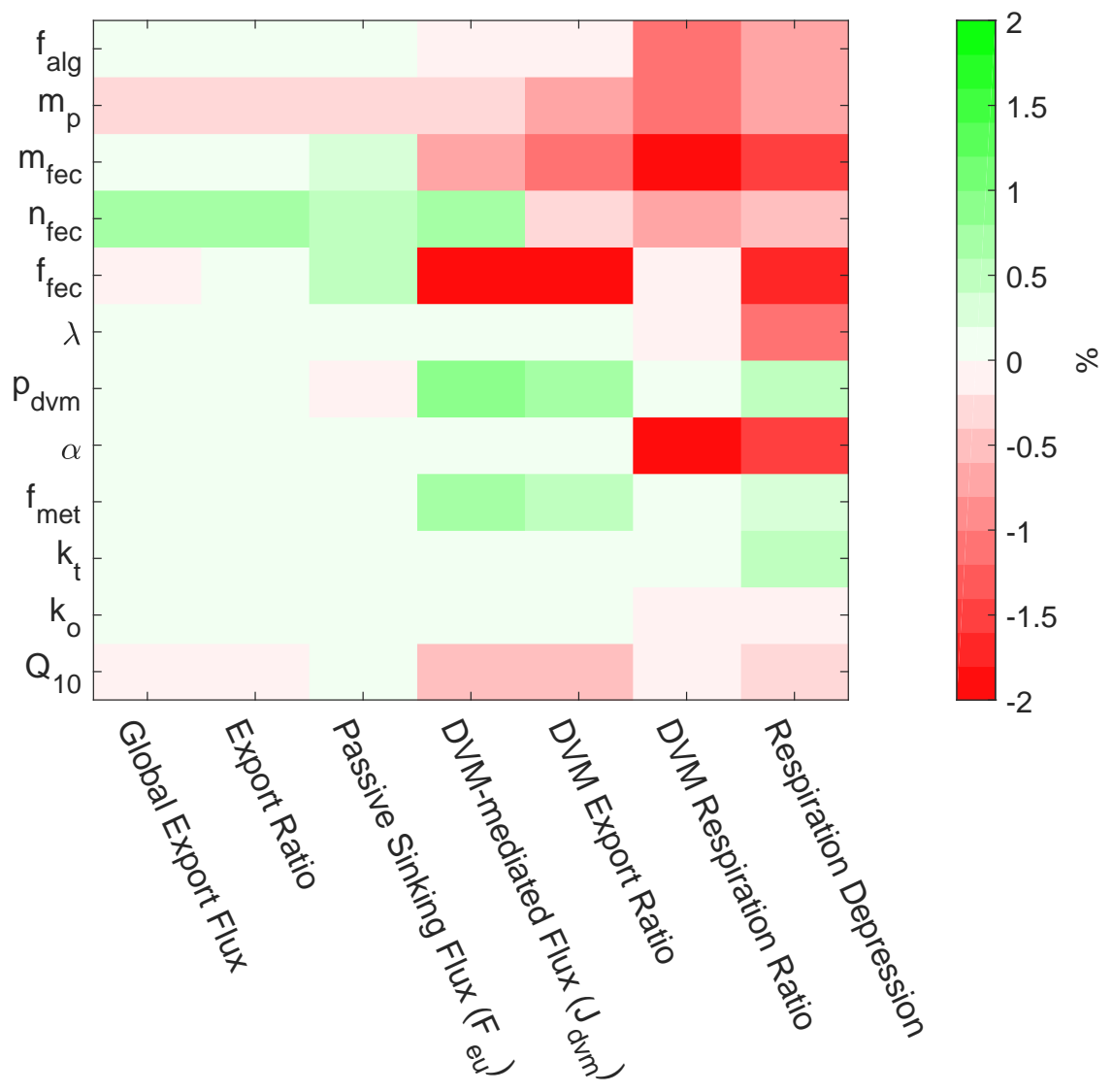

Figure 2-8: Results of the sensitivity analysis. Each box represents the percent change in the model output based on a $1 \%$ increase in the parameter value. Parameter rows with zero sensitivity on all the output metrics have been excluded. 
observations and in situ measurements show a succession from larger diatom cells in the early spring to a more diverse community of smaller cells that dominate during the summer. However significant differences are found between the model estimates of mean large zooplankton biomass integrated over the euphotic zone $\left(3.5 \pm 0.7 \mathrm{mgC} \mathrm{m}^{-3}\right)$ and in situ measurements made at BATS. (Madin et al., 2001) measured zooplankton biomass in the top $200 \mathrm{~m}$ from 1994-1998 and estimated a mean value of $1.16 \pm 0.19 \mathrm{mgC} \mathrm{m}^{-3}$. The discrepancy between model estimate and observation could indicate that the zooplankton mortality terms are poorly defined for this location. However, the general lack of strong seasonal patterns in zooplankton biomass, besides the small peak in early spring, was consistent with observations (Madin et al., 2001; Steinberg et al., 2001). Model estimates of the mean total export flux $\left(18.8 \mathrm{mgC} \mathrm{m}^{-2} \mathrm{~d}^{-1}\right)$ and the mean export ratio (0.07) at BATS were reasonable compared to long term measurements made at the time series location from 1989-1997, where (Steinberg et al., 2001) report mean values of $25.8 \mathrm{mgC} \mathrm{m}^{-2} \mathrm{~d}^{-1}$ and 0.06 respectively. Table 2.4 provides a summary of these comparisons.

We also compared the modeled large zooplankton biomass across the global domain to estimates of global mesozooplankton biomass made by (Moriarty and O'Brien, 2013) using the Coastal and Oceanic Plankton Ecology, Production, and Observation Database (COPEPOD). The COPEPOD database combines mesozooplankton biomass measurements from 110 studies and converts the data to reflect the $333 \mu \mathrm{m}$ mesh size fraction over the depth range from 0-500 $\mathrm{m}$. The model predicts a global mean large zooplankton biomass of $2.8 \mathrm{mgC} \mathrm{m}^{-3}$ with a standard deviation of $0.9 \mathrm{mgC} \mathrm{m}^{-3}$, while COPEPOD has a global mean of $5.9 \mathrm{mgC} \mathrm{m}^{-3}$, a median of $2.7 \mathrm{mgC} \mathrm{m}^{-3}$, and a standard deviation of $10.6 \mathrm{mgC} \mathrm{m}^{-3}$ (Moriarty and O'Brien, 2013). We can infer from this comparison that the model does a good job of describing the central tendency of the global distribution of zooplankton biomass, but falls short of accurately describing the regional variability because it does not characterize anomalously high values of zooplankton biomass that have been observed. The spatial patterns in both the modeled zooplankton biomass and observations from COPEPOD show elevated values in regions of high NPP, most importantly in shelf waters around the globe. However, the COPEPOD data show very high concentrations of zooplankton north of $60^{\circ} \mathrm{N}$, particularly in the Bering Sea (Moriarty and O'Brien, 2013). Unfortunately, the model does a poor job of representing plankton ecosystems north of $60^{\circ} \mathrm{N}$ due to a lack of satellite coverage over the seasonal cycle. The limited spatial range of the model may partially explain why the model does not do a good job of 
Table 2.4: Summary of the comparisons between model output at BATS and in situ observations made at the Bermuda Atlantic Time Series scientific station. Observation means and standard deviations (SD) are taken from reviews by (Steinberg et al., 2001) and (Madin et al., 2001).

\begin{tabular}{||c|c|c|c||}
\hline Statistic & $\begin{array}{c}\text { Model Mean } \\
(\mathrm{SD})\end{array}$ & $\begin{array}{c}\text { Observation Mean } \\
(\mathrm{SD})\end{array}$ & $\begin{array}{c}\text { Percent Difference } \\
(\%)\end{array}$ \\
\hline $\begin{array}{c}\text { Zooplankton Biomass } \\
\left(\mathrm{mgC} \mathrm{m}^{-2}\right)\end{array}$ & $3.5(0.68)$ & $1.16(0.19)$ & 100.6 \\
$\begin{array}{c}\text { Total Export Flux } \\
\left(\mathrm{mgC} \mathrm{m}^{-2} \mathrm{~d}^{-1}\right)\end{array}$ & $18.8(5.2)$ & $25.8(3.8)$ & 31.4 \\
Export Ratio & $0.07(0.03)$ & $0.06(0.01)$ & 15.4 \\
\hline
\end{tabular}


describing the high end of the distribution of zooplankton biomass.

Finally, we compared model estimates of the DVM-mediated export flux to empirical observations (Fig. 2.9). The active respiratory flux of carbon as a result of zooplankton DVM has been quantified in a number of locations based on vertical migrant biomass and estimates of metabolic rates (Isla et al., 2015). Suitable data for comparison to the model exists for BATS (Steinberg et al., 2000), the equatorial Pacific (Zhang and Dam, 1997), ALOHA (Al-Mutairi and Landry, 2001), the north Atlantic (Isla and Anadon, 2004; Longhurst et al., 1990), the Canary Islands (Hernández-León et al., 2001), and the California Current (Aumont et al., 2018). Overall, the model does a good job of capturing broad patterns in the magnitude of the DVM-mediated export flux. To first order, the model predicts higher DVM export fluxes at the stations where the active respiratory flux has been observed to be larger. However, plotting the data does reveal the model is consistently overestimating the DVM export flux. There are a number of important parameters which have a linear relationship with model outputs (Fig. 2-8) which may be contributing to the discrepancy between the model and observations. While we made no attempt at optimization for this misfit, as it is outside the scope of this contribution, small changes to parameter values such as $p_{d v m}$ would likely bring the model estimates more in line with observations. Only rarely have numerical models been used to quantify the DVM-mediated export fluxes on global scales. A recent study by (Aumont et al., 2018) created a synthesis of the NEMO-PISCES and Apex Predators ECOSystem models tuned with empirical observations of zooplankton biomass to estimate DVM-mediated export.

Elevated contributions of DVM activity to export and mesopelagic biogeochemistry in the subtropical latitudes results from the combined effect of multiple contributing physical and biological factors. Reduced chlorophyll concentrations at the surface and high incident irradiance levels in subtropical latitudes means that the target isolume is deeper in the water column, resulting in increased migration depths. Additionally, higher values of $\mathrm{NPP}_{n} / \mathrm{NPP}_{m}$ results in smaller fluxes of sinking particles with respect to other sources of export, including active transport by DVM, since nano-phytoplankton do not sink out of the euphotic zone. Increased temperature also modulates biogeochemical activity and plays a role in determining the effect of DVM. Higher temperature decreases the characteristic remineralization length scale and shoals the remineralization profile (Laufkötter et al., 2017; Marsay et al., 2015), making the localized input of DIC into the twilight zone by DVM a larger perturbation. In contrast, a steeper temperature vertical gradient 


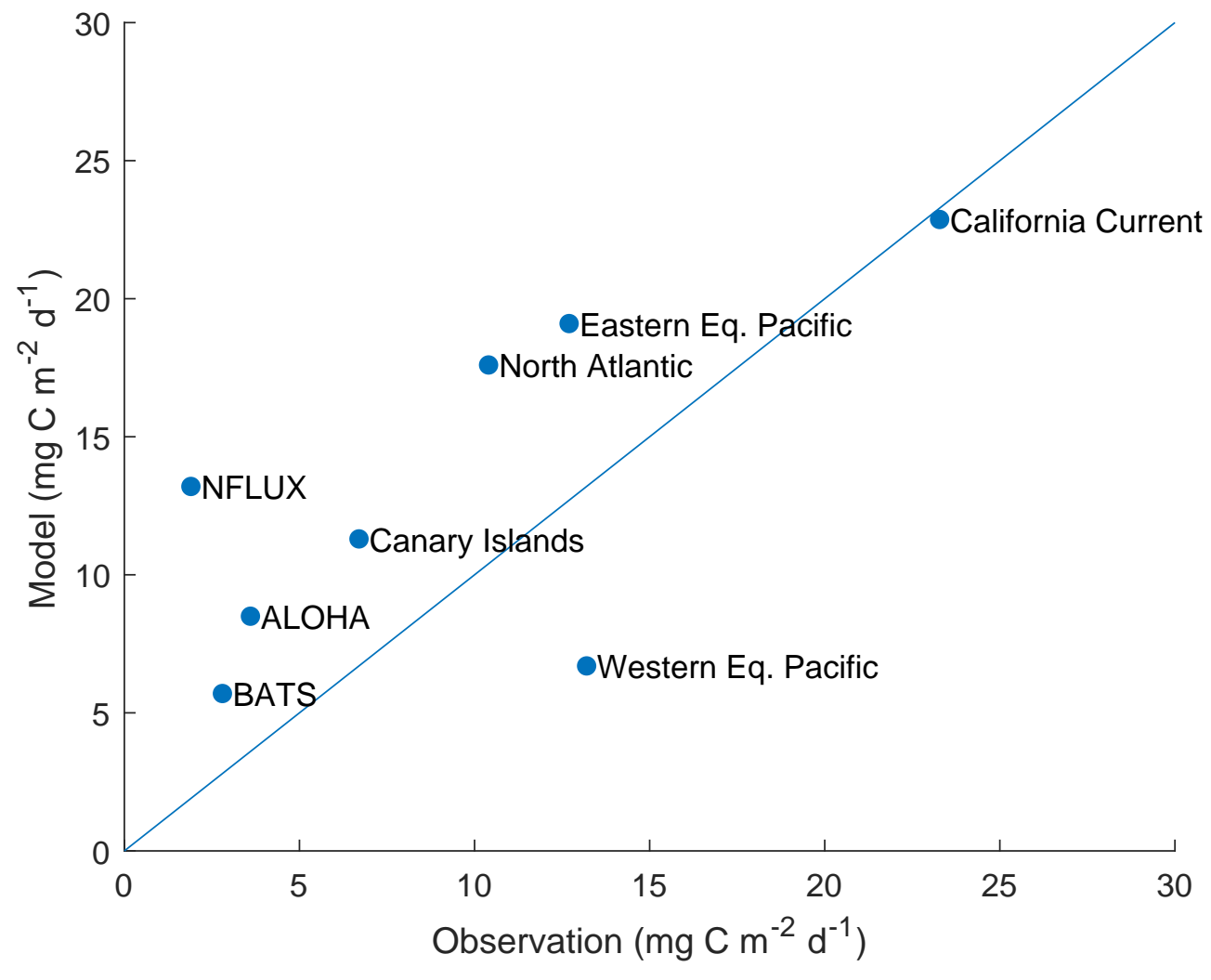

Figure 2-9: Comparison of model estimates of the DVM-mediated export flux to empirical observations at a variety of locations. A 1:1 line is included for reference. 
reduces $p_{m e t}$, due to the increased temperature difference between the surface and the euphotic zone (Eq. 2.11), and tends to make the fraction of absorbed carbon metabolized in the twilight zone $\left(J_{m e t}\right)$ smaller. We can infer from the increased values of respiration depression in the subtropics (Fig. 2-7) that the shoaling of the sinking POC remineralization profile has a greater effect than the reduction of $J_{\text {met }}$. A larger respiration depression indicates that carbon will generally be deposited deeper in the water column as a result of DVM activity, into water that is older and further removed from the atmosphere (Kwon et al., 2009).

Overall, the contribution of DVM activity to DIC production and oxygen utilization in the twilight zone was larger than its contribution to the vertical POC flux, indicating that the impacts of DVM are primarily respiratory in nature. One important result of increased DVM is an oxygen utilization profile that does not decrease monotonically with depth. While the remineralization of sinking particles does decrease monotonically over the water column, this is only one of the processes that contribute to total oxygen utilization in the twilight zone. Respiration rates by vertically migrating zooplankton account for a significant portion of the total oxygen utilization. In locations where DVM is an important contributor to water column DIC production, the presence of migrating zooplankton should produce a significant oxygen utilization spike at depth. The distribution over depth and the maximum magnitude of this spike depends on the parameterized width of the Gaussian used to distribute estimated zooplankton metabolism. We used observations of the thickness of migrating zooplankton layers to parameterize the model (Bianchi and Mislan, 2016), but did not allow for these parameters to change over the model domain. In real populations, regional differences in the representation by different taxa and intraspecific variations may result in a source of DIC production that is either more narrow or more diffuse than presented here. For smaller-scale applications of the model, instead of parameterizing the distribution of zooplankton around a mean depth, it should be possible to define the depth and thickness of the migrating zooplankton layer using acoustic data. Regardless, model results imply that respiration by migrating zooplankton makes up a significant proportion of total water column oxygen utilization and DVM should have important impacts on the magnitude and vertical structure of the oxygen utilization profile within the twilight zone.

The model makes a number of simplifying assumptions concerning remineralization and vertical migrations in the twilight zone. First, we have chosen to treat sinking POC as a single pool of 
carbon that is remineralized using an exponential decay model. Alternative parameterizations exist, however, which include additional information about variable remineralization rates, sinking speeds, and particle compositions. Two popular alternatives are the Martin Curve (Martin et al., 1987) and the Ballast Hypothesis (Armstrong et al., 2002). The Martin Curve model uses a normalized power function, which, compared to the exponential decay model used here, is equivalent to incorporating remineralization rates that decline with depth or sinking speeds that increase with depth. The Ballast Hypothesis model assumes that certain minerals associated with the POC flux (including silicate, calcium carbonate, and dust) serve to increase the total export flux by increasing sinking speeds and protecting POC from microbial remineralization. Despite the conceptual differences between these three models, an evaluation of the performance of the three models in different biogeochemical regimes by (Gloege et al., 2017) found that the exponential model performs equally well to the Martin Curve and the Ballast Hypothesis models over most of the twilight zone. Below $1000 \mathrm{~m}$, the exponential decay model tended to underestimate the export flux because it does not account for variable remineralization length scales (Gloege et al., 2017). However, the depth scale of the model was set by the depth of DVM, which was shallow enough that considerations about sinking particles below one kilometer were not relevant. The exponential decay model also has the advantage of increased simplicity with fewer parameters compared to alternative remineralization models, which is advantageous for the exploratory nature of this study.

Another important simplifying assumption of this model is the representation of diel vertical migration. In the ocean, many populations of zooplankton and fish participate in vertical migrations over many different and overlapping depth ranges and some of these populations do not ever enter the euphotic zone. This system of interconnected vertical migrations that passes POC down into the twilight zone through depth-tiered populations has been described as Vinogradov's Ladder (Vinogradov, 1962) or the Bucket Brigade Hypothesis (Ochoa et al., 2013). The model described here integrates this system of vertical migrations into a single event that transports carbon from the euphotic zone to a target depth in the twilight zone, both for simplicity of illustration and due to a lack of data constraints. While the food web model of the euphotic zone provides constraints on grazing rates of zooplankton populations that spend at least some fraction of their time in the surface ocean, there is a lack of adequate data to fully parameterize the biological complexity of the bucket brigade hypothesis. The model instead represents an idealized scenario in which a single 
zooplankton migration is represented for the entire migrant community. The results presented here should be interpreted in the context of comparing model simulations which included DVM behavior to control runs which did not. As a result, interpretation of these results should be caveated by the acknowledgement that in real ecosystems, migration depth will vary significantly by taxa, including taxa whose diel migrations never go above the euphotic zone depth. As a result, the spike of DIC production and oxygen utilization seen in the model results is likely to be a more diffuse source spread out across the twilight zone.

The modeled global export flux is more robust to changes in the parameter values than the relative contribution of the passive sinking flux and the DVM-mediated flux to total export. This is not surprising, considering that the model represents multiple pathways that all sum to total export. However, the magnitude of the DVM-mediated export flux is highly sensitive to at least three parameters that have relatively high uncertainty in the model: $f_{f e c}, p_{d v m}$, and $f_{m e t}$. In this study, we assumed that these parameters were constant across the global domain, but variations within and among zooplankton communities is expected to generate both spatial and temporal variability (Steinberg and Landry, 2017). For example, the proportion of zooplankton participating in DVM can vary anywhere from no DVM to a migration of nearly the entire community (Isla et al., 2015; Klevjer et al., 2016; Putzeys and Hernández-Leon, 2005; Takahashi et al., 2009). This variability will exist both spatially between grid points and temporally over the seasonal cycle. The model also relies on very rough estimates of trophic efficiency to estimate the scaling factor of $n_{f e c}: m_{f e c}$ and variability in species composition and life history strategy will result in regional differences in this ratio. Additional work is needed to quantify the variability of these parameters in order to understand the effect of regional differences in zooplankton community characteristics on DVM-mediated carbon export. Translation between the parameters used in this model and traditional physiological measurements made by laboratory experiments is straightforward. For example, the assimilation efficiency of the large zooplankton size class is equation to $1-m_{f e c}$ and the gross growth efficiency of micro-grazers is equal to the ratio of $n_{f e c} / m_{f e c}$.

In addition to parameter uncertainty, the results may also be affected by structural uncertainty (e.g. additional trophic levels, mixotrophy). For example, additional trophic levels of zooplankton below the migrators would substantially diminish the impact of DVM due to further respiratory loss of grazed carbon at the surface. We have also designed the model such that none of the zooplankton 
mortality term contributes to the carbon export flux. The treatment of zooplankton mortality terms varies considerably in biogeochemical models, ranging from complete remineralization in the surface ocean to complete routing of the loss terms to the export flux (Laufkötter et al., 2016). We have assumed that biomass lost through zooplankton mortality is routed upward to higher trophic levels or remineralized in the euphotic zone, but acknowledge that this assumption is likely to reduce our overall estimate of the global carbon export flux. In general, we have opted to use a simple food web model to investigate broad impacts of implementing DVM behaviors into an export model. We encourage future work that establishes how increasingly complex and realistic food web models influence the results presented here.

The model suggests that zooplankton respiration in the twilight zone is an important component of the export flux of the biological pump. As a result, empirical studies which quantify the export flux by measuring the sinking of POC throughout the water column (Eppley and Peterson, 1979; Henson et al., 2011) may be underestimating the export ratio. The pathway by which zooplankton graze phytoplankton biomass in the surface ocean and subsequently metabolize a portion of that biomass at depth is a significant component of the biological pump which completely circumvents the vertical POC flux. An accurate portrayal of the biological pump, both conceptually and quantitatively, should include the action of zooplankton DVM and its effect on both particle export and water column biogeochemistry (Buesseler and Boyd, 2009).

It has long been acknowledged that zooplankton vertical migrations play an important role in the biological pump and the biogeochemistry of the twilight zone. Respiratory models of the biological pump that include DVM have estimated that the flux of carbon mediated by migrating zooplankton is 13 to $58 \%$ of the passive sinking flux (Longhurst et al., 1990) and accounts for up to $50 \%$ of the production of DIC in the twilight zone (Bianchi et al., 2013b). Results from our model are consistent with these observations and show significant contributions by zooplankton DVM to both carbon export and respiration in the twilight zone. However, despite solid evidence that DVM is a significant contributor to carbon export, the phenomenon is often excluded from global models of the biological pump, perhaps in part due to the difficulty in parameterizing the process. This study provides a valuable first step in quantifying the global variability in DVM-mediated contributions to the biological pump. Additionally, and equally important, it provides a relatively straightforward parameterization of zooplankton DVM that could be implemented in a global model as a basis for 
future investigations. 


\section{Chapter 3}

\section{The Kill-the-Winner functional response: Coexistence dynamics and synergistic grazing}

\subsection{Introduction}

Phytoplankton communities commonly consist of many species living together in an apparently homogeneous environment and competing for a small number of limiting resources. This coexistence perplexed ecologists in the middle of the twentieth century because the current understanding of ecology led them to believe that the strongest competitor should exclude all others (Hardin, 1960). Hutchinson (1961) termed this disparity between observations and ecological theory the "paradox of the plankton." Beginning in the 1960s and 70s, a number of ecological mechanisms were identified that promote species coexistence and counteract the competitive exclusion principle. These include fluctuating environmental conditions that prevent the community from reaching equilibrium (Hutchinson, 1941), multiple limiting resources (Tilman, 1982), niche partitioning and intra-specific competition (Haydon, 1994), and density-dependent mortality, such as virus induced mortality (Thingstad, 2000) or prey-selective grazing. Density-dependent mortality falls within the category of 'equalizing mechanisms' to sustain diversity (Song et al., 2019). In particular, it has been shown that grazers switching between multiple prey species can mediate coexistence between 
competitors and increase diversity in food webs (Adjou et al., 2012; Prowe et al., 2012; Vallina and Le Quéré, 2011).

Mathematical models have proven to be a useful tool in studying how grazing can modulate competitive interactions and mediate coexistence (Armstrong, 1979; Holt et al., 1994; Leibold, 1996; Roughgarden and Feldman, 1975). In models, grazing is determined by the functional response, the relationship between the amount of a resource and the rate at which that resource is consumed by an individual grazer. Holling (1959) described three types of functional responses that have become standard in ecological models: linear, concave down or saturating hyperbolic, and sigmoid (commonly referred to as Types I, II, and III). These classic functional responses can be derived from first principles based on the attack rate and handling time of the grazer for a given resource (in the case of Types I and II) or defined based on experimental data (in the case of Type III). However, functional responses that include multiple resources are more complicated because the relationship between consumption rate and resource density may be different for different resources. These relationships are further complicated by the fact that the consumption rate of one resource may be affected by the abundance of other available resources (Kiørboe et al., 1996; Strom and Loukos, 1998).

Gentleman et al. (2003) provide a review of functional responses that have been used in the literature to describe zooplankton grazing on multiple phytoplankton types. Most of these functional response models are phenomenological in nature; rather than being derived from first principles, they are designed in such a way that they replicate a desired behavior. As a result, they sometimes include biologically unrealistic characteristics (Gentleman et al., 2003). One example of this is the phenomenon of antagonistic grazing, in which a zooplankter has a higher total consumption rate when feeding on a single phytoplankton type than it would when the same total biomass is divided among many different phytoplankton types (Tilman, 1982). To avoid antagonistic feeding, Vallina et al. (2014) developed an alternative formulation of distributed grazing, the Kill-theWinner (KTW) functional response, which includes both zooplankton preference and switching. Since its publication, the KTW functional response has become a popular way to include switching behavior in a variety of models (Baudrot et al., 2016; Cadier et al., 2017; Chen et al., 2019; Egilmez and Morozov, 2016; Guyennon et al., 2015; Nissen et al., 2018; Smith et al., 2016; Tanioka and Matsumoto, 2018; Vallina et al., 2017; Ward and Follows, 2016). 
In the following study, we examine the dynamical effects of the KTW functional response in simple food web models. Our main findings are three-fold. First, with a simple diamond-shaped food web model, we establish that the KTW functional response is consistent with widely accepted ecological results regarding grazer-mediated coexistence between competing phytoplankton types. Confirmatory results such as this are especially important when dealing with phenomonological functional responses, since functions that may appear nearly indistinguishable can produce very different dynamics (Fussmann and Blasius, 2005). Second, this analysis, which intentionally does not include ocean circulation or size structure within the phytoplankton, helps to elucidate the fundamental dynamics of the KTW functional response on the scale of competition between individual phytoplankton types and provides context for previous results showing that using the KTW functional response increases phytoplankton diversity on a global scale (Vallina et al., 2014b). Third, we describe one characteristic of the KTW functional response that generates biologically improbable effects under certain conditions. We call this effect synergistic grazing and discuss its effect on model dynamics.

\subsection{Model Construction and Analysis}

\subsubsection{Preference and switching in the KTW functional response}

Throughout this paper, we discuss two grazer characteristics that affect the competitive interaction between phytoplankton types: preference and switching. A zooplankter is said to exhibit preference for a phytoplankton type when the proportion of that type in its diet exceeds the proportion of that type in the environment (Cock, 1978; Hassell, 1978; Roughgarden and Feldman, 1975). Zooplankton preference may arise from differential attack rates or rejection of less desirable phytoplankton types (Cock, 1978). A zooplankter is said to exhibit switching when the proportion of a phytoplankton type in its diet changes from less than expected to greater than expected as the proportion of that phytoplankton type in the environment increases (Hassell, 1978). Switching represents some behavioral change in the zooplankton that occurs in response to a variable phytoplankton community. Such responses include changing feeding strategies or learning how to capture or handle a particular phytoplankton type more efficiently (Hassell, 1978; Vallina et al., 2014b). In Appendix A of their paper, Vallina et al. (2014) explore the relationship between the phenomena 
of preference and switching and a zooplankter's attack rate on a given phytoplankton type. It has previously been shown that preference and switching can promote coexistence between competing phytoplankton species or size classes in circumstances that would otherwise lead to competitive exclusion (Armstrong, 1994; Vallina et al., 2014a; Ward et al., 2014).

The KTW functional response includes both preference and switching (Vallina et al., 2014b). It defines the per capita grazing rate on phytoplankton type $P_{i}$, in a community of $n$ types, as

$$
G_{i}\left(P_{1}, P_{2}, \ldots, P_{n}\right)=g_{\max } \frac{\rho_{i} P_{i}^{\alpha}}{\sum_{j=1}^{n} \rho_{j} P_{j}^{\alpha}} \frac{\sum_{j=1}^{n} \rho_{j} P_{j}}{k_{s a t}+\sum_{j=1}^{n} \rho_{j} P_{j}}
$$

$G_{i}$ has two components. One component, $g_{\max } \sum_{j=1}^{n} \rho_{j} P_{j} /\left(k_{s a t}+\sum_{j=1}^{n} \rho_{j} P_{j}\right)$, represents the total grazing rate as it depends upon the total preference-weighted biomass of the $n$ phytoplankton types. It takes the form of a typical Holling Type II functional response with a maximum consumption rate $g_{\max }$ and half-saturation constant $k_{\text {sat }}$. The preference for phytoplankton type $i$ is $\rho_{i}$. If the zooplankter has the same preference for all phytoplankton types then all the values of $\rho_{i}$ are equal. If the zooplankter has different preferences for the prey, then the values of the $\rho_{i}$ will not all be equal; a larger value of $\rho_{i}$ indicates a stronger preference for type $i$.

The other component, $\rho_{i} P_{i}^{\alpha} / \sum_{j=1}^{n} \rho_{j} P_{j}^{\alpha}$, specifies the portion of the total grazing rate that is applied to the ith phytoplankton type. The value of $\alpha$ controls the switching behavior. If the zooplankter does not exhibit switching, then $\alpha=1$. When $\alpha>1$, the zooplankter exhibits some degree of switching with larger values of $\alpha$ corresponding to stronger switching behaviors. For very large values of $\alpha$, the grazing pressure is concentrated entirely on the most abundant phytoplankton type. Previous work has evaluated the effect of the KTW functional response on phytoplankton diversity for values of $\alpha=[1,3]$ and found a positive relationship between the value of $\alpha$ and phytoplankton diversity (Smith et al., 2016). This work, which used a continuous traitdistribution model for a phytoplankton community subject to environmental disturbance, focused on the adaptive dynamics that play out on evolutionary timescales (as opposed to the ecological timescales we consider here).

If a zooplankter exhibits neither preference nor switching behavior, then the proportion of a phytoplankton type in the zooplankter's diet will be equal to that type's proportion in the environment (Fig 3-1, solid black line). If a zooplankter exhibits preference for a phytoplankton type, but not 
switching, then the proportion of a phytoplankton type in the zooplankter's diet will be higher than its proportion in the environment (Fig 3-1, solid blue line). If the zooplankter exhibits switching, then the proportion of a phytoplankton type in the zooplankter's diet will change from less than expected to greater than expected (relative to the case where the zooplankter has no preference or switching) as the proportion of that type in the environment increases (Fig 3-1, dashed lines). Switching to the more common phytoplankton type occurs at a lower proportional abundance if the zooplankter has a preference for that type, and at a higher proportional abundance if it has a preference for other types.

\subsubsection{The diamond-shaped food web model}

To better understand how KTW affects the dynamics of phytoplankton competition, we included it in a diamond-shaped food web model with one nutrient resource $(N)$, two competing phytoplankton types $\left(P_{1}\right.$ and $\left.P_{2}\right)$, and a single zooplankton species $(Z)$ that feeds upon both phytoplankton (Fig. 3-2). The model assumes a constant and well-mixed environment, such as the mixed layer in a pelagic water column. Nutrients are supplied to the mixed layer through vertical mixing with deeper waters. This influx water has a nutrient concentration $N_{0}$ and is supplied at a rate $D$. Nutrients, phytoplankton, and zooplankton are mixed out of the water column at the same rate $D$. The implicit assumption here is that $Z$ represents grazers similar to microzooplankton, which have vertical swimming speeds that are too small to prevent being mixed out of the surface layer. The maximum growth rates of $P_{1}$ and $P_{2}$ are given by $\mu_{1}$ and $\mu_{2}$. Nutrient uptake by the phytoplankton follows Monod kinetics with half-saturation constants $k_{1}$ and $k_{2}$. The phytoplankton non-grazing mortality rate is given by $m_{p}$ and the mortality of the zooplankton is given by $m_{z} \cdot \gamma$ is the growth efficiency of the zooplankton. The dynamics of this food web are given by

$$
\begin{aligned}
\frac{d N}{d t} & =D\left(N_{0}-N\right)-\mu_{1} \frac{N}{k_{1}+N} P_{1}-\mu_{2} \frac{N}{k_{2}+N} P_{2} \\
\frac{d P_{1}}{d t} & =\mu_{1} \frac{N}{k_{1}+N} P_{1}-G_{1}\left(P_{1}, P_{2}\right) Z-\left(m_{p}+D\right) P_{1} \\
\frac{d P_{2}}{d t} & =\mu_{2} \frac{N}{k_{2}+N} P_{2}-G_{2}\left(P_{1}, P_{2}\right) Z-\left(m_{p}+D\right) P_{2} \\
\frac{d Z}{d t} & =\gamma \sum_{i=1}^{2} G_{i}\left(P_{1}, P_{2}\right) Z-\left(m_{z}+D\right) Z
\end{aligned}
$$




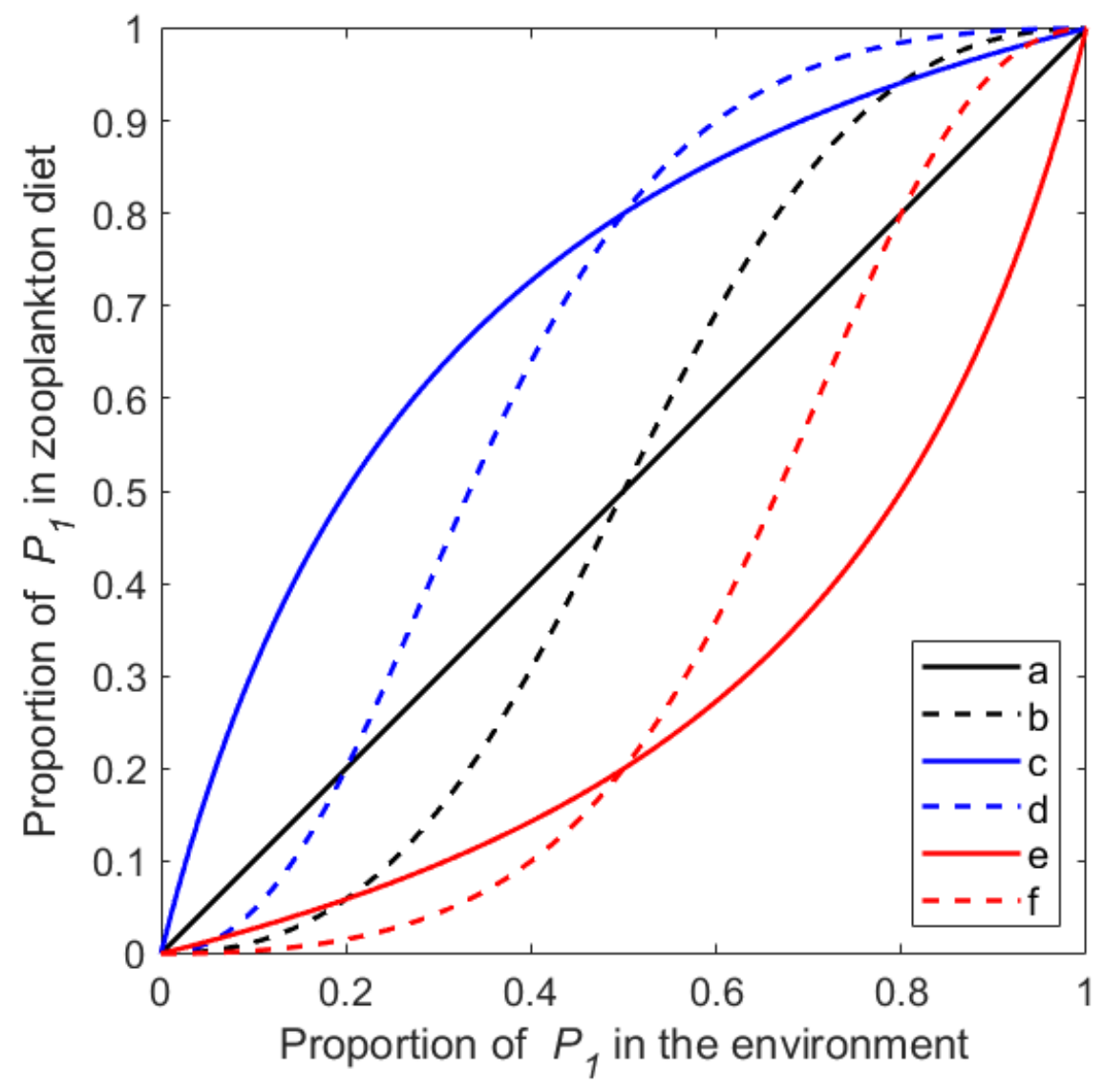

Figure 3-1: The proportion of $P_{1}$ in a zooplankter's diet as a function of the proportion of $P_{1}$ in the environment based on Eq. 3.1 and assuming two phytoplankton types. The different curves show different combinations of preference and switching: (a) neither preference nor switching $\left(\rho_{1}=\right.$ $\left.\rho_{2}, \alpha=1\right)$, (b) switching without preference $\left(\rho_{1}=\rho_{2}, \alpha>1\right)$, (c) preference for $P_{1}$ without switching $\left(\rho_{1}>\rho_{2}, \alpha=1\right)$, (d) preference for $P_{1}$ with switching $\left(\rho_{1}>\rho_{2}, \alpha>1\right)$, (e) preference for $P_{2}$ without switching $\left(\rho_{1}<\rho_{2}, \alpha=1\right)$, (f) preference for $P_{2}$ with switching $\left(\rho_{1}<\rho_{2}, \alpha>1\right)$. 
In the absence of any grazing (i.e., when $Z=0$ ), the phytoplankton type that is the better competitor for nutrients will eventually exclude the other phytoplankton type in model (3.2)-(3.5) (Hsu, 1978). We can define a metric for the competitive ability of the $i$-th phytoplankton type using classical R-star theory (Tilman, 1977)

$$
R_{i}^{*}=\frac{k_{i}\left(D+m_{p}\right)}{\mu_{i}-\left(D+m_{p}\right)}
$$

If $R_{i}^{*}<R_{j}^{*}$, then $P_{i}$ is a stronger competitor for nutrients than $P_{j}$ and $P_{i}$ will drive $P_{j}$ to extinction. We chose $\mu_{1}>\mu_{2}$ and $k_{1}<k_{2}$ to satisfy the criteria that $P_{1}$ is the stronger competitor (Fig. 3-2).

Zooplankton preference and switching may allow for coexistence between $P_{1}$ and $P_{2}$ in situations where, in the absence of the zooplankton, $P_{1}$ would drive $P_{2}$ to extinction. Consider the scenario where $P_{2}=0$; the equilibrium point of the linear food chain composed of $N, P_{1}$, and $Z$ is given by

$$
\begin{aligned}
N^{*} & =\frac{1}{2 D}\left[-\left(\mu_{1} P_{1}^{*}+k_{1} D-N_{0} D\right)+\sqrt{\left(\mu_{1} P_{1}^{*}+k_{1} D-N_{0} D\right)^{2}+4 k_{1} D^{2} N_{0}}\right] \\
P_{1}^{*} & =\frac{\left(m_{z}+D\right) k_{s a t}}{\rho_{1}\left(\gamma g_{\max }-m_{z}-D\right)}, \\
Z^{*} & =\frac{k_{\text {sat }}+\rho_{1} P_{1}^{*}}{\rho_{1} g_{\max }}\left(\frac{\mu_{1} N^{*}}{k_{1}+N^{*}}-m_{p}-D\right) .
\end{aligned}
$$

Near this equilibrium point, the dynamics of $P_{2}$ are given by the linearization of model (3.2)-(3.4). The population of type $P_{2}$ will grow (or decay) according to the invasion growth rate $r_{2}$, which represents the per capita growth rate of $P_{2}$ when $P_{2} \approx 0$ :

$$
r_{2}=\left.\frac{1}{P_{2}} \frac{d P_{2}}{d t}\right|_{\left(N^{*}, P_{1}^{*}, 0, Z^{*}\right)}= \begin{cases}\frac{\mu_{2} N^{*}}{k_{2}+N^{*}}-m_{P}-D-\frac{\rho_{2} g_{\max } Z^{*}}{k_{s a t}+\rho_{1} P_{1}^{*}} & \text { if } \alpha=1 \\ \frac{\mu_{2} N^{*}}{k_{2}+N^{*}}-m_{P}-D & \text { if } \alpha>1\end{cases}
$$

If $r_{2}>0$, then $P_{2}$ can successfully invade the system.

Note that the term $\frac{\rho_{2} g_{\max } Z^{*}}{k_{s a t}+\rho_{1} P_{1}^{*}}$ in Eq. 3.9 is always positive, so the growth of a phytoplankton type when its density is near zero is strictly greater when the zooplankton grazer displays switching. Zooplankton switching behavior, therefore, represents a kind of density-dependent mortality that provides a refuge for phytoplankton at low densities. Eq. 3.9 suggests that there exist some values of $\rho_{i}$ and $\alpha$ such that $r_{2}>0$ even when $R_{2}^{*}<R_{1}^{*}$. Stated differently, a weaker phytoplankton competitor should be able to invade the equilibrium of a stronger phytoplankton competitor under 
a)

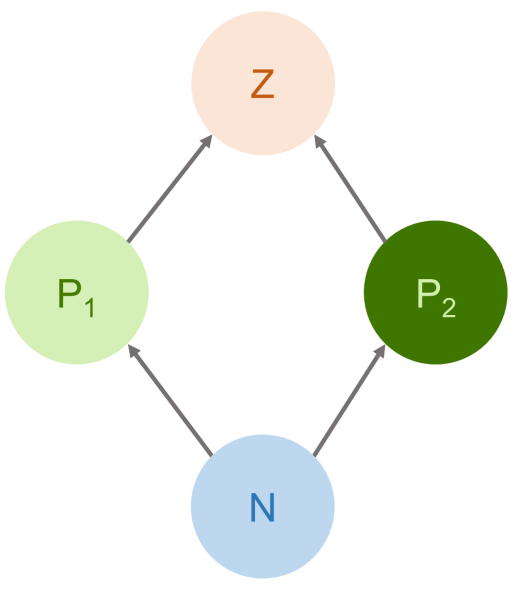

b)

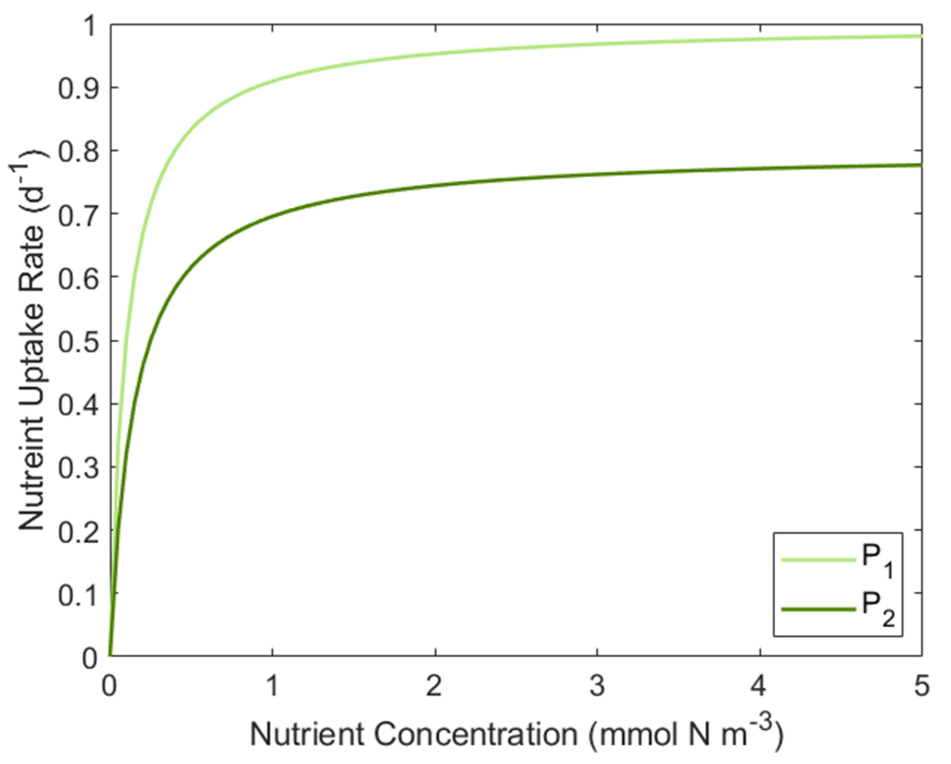

Figure 3-2: (a) The structure of the diamond-shaped food web model and (b) the Michaelis-Menten curves for the nutrient uptake of $P_{1}$ and $P_{2}$. 
certain grazing conditions. We explored this hypothesis further by simulating the diamond-shaped food web model in each of six test cases which cover different combinations of preference and switching (Table 3.1).

For each case, we simulated model (3.2)-(3.5) over a range of input nutrient concentrations $\left(N_{0}\right)$ and mixing rates $(D)$ using random initial conditions drawn from uniform distributions. We observed four possible asymptotic outcomes: (1) $P_{1}>0, P_{2}=0$, and $Z=0$, (2) $P_{1}>0, P_{2}=0$, and $Z>0$, (3) $P_{1}=0, P_{2}>0$, and $Z>0$, or (4) $P_{1}>0, P_{2}>0$, and $Z>0$. Each of these outcomes was characterized by a stable equilibrium. For these simulations, $P_{1}$ was the stronger competitor and $P_{2}$ was the weaker competitor.

For Cases 1 and 3, it can be shown that $r_{2}<0$ for all positive parameter values (see Appendix S1), and therefore $P_{1}$ will always drive $P_{2}$ to extinction. We found that coexistence is possible in the diamond-shaped food web model if (1) the zooplankton have a preference for the stronger competitor or (2) the zooplankton exhibit switching (Table 3.1). The range of environmental conditions (i.e., nutrient inputs and mixing rates) that allows for coexistence in Case 2, where zooplankton exhibit preference only, is relatively small, indicating that coexistence mediated by preference alone is rare and represents a delicate balance between zooplankton preference and the growth rates and nutrient affinities of the competing phytoplankton types (Fig. 3-3). In contrast, for Cases 4-6, where zooplankton exhibit switching, coexistence occurs over a wider range of environmental conditions, indicating that switching is a robust mechanism for promoting coexistence. Finally, only in Case 2 can the weaker phytoplankton type drive the stronger phytoplankton type to extinction; this occurs when the dilution rate $D$ and the inflowing nutrient concentration $N_{0}$ are sufficiently large (Fig. 33). The simulation results were consistent with our theoretical predictions based on Eq. 3.9. The $r_{2}=0$ curve, where the invasion growth rate for the weaker competitor changes sign, corresponds to the boundary between the region of the parameter space where the model is asymptotically stable with $P_{2}=0$ and the region where stable coexistence between $P_{1}$ and $P_{2}$ occurs (Fig. 3-3).

\section{Synergistic grazing in the diamond-shaped food web model}

We will now address the existence of synergistic grazing in the KTW functional response. Mathematically, synergistic grazing occurs when $\frac{d G_{i}}{d P_{j}}>0$ for $i \neq j$. In other words, the specific grazing rate on $P_{1}$ increases as the density of $P_{2}$ increases (Fig. 3-4). Synergistic grazing only occurs when 
Table 3.1: Summary of model test cases in the diamond-shaped food web model ${ }^{a, b}$

\begin{tabular}{||c|c|c|c|c|c|c||}
\hline Case & Preference & $\rho_{1}$ & $\rho_{2}$ & Switching & $\alpha$ & Possible Coexistence \\
\hline 1 & equal preference for $P_{1}$ and $P_{2}$ & 1 & 1 & no & 1 & no \\
2 & higher preference for $P_{1}$ & 1 & 0.5 & no & 1 & yes \\
3 & higher preference for $P_{2}$ & 0.5 & 1 & no & 1 & no \\
4 & equal preference for $P_{1}$ and $P_{2}$ & 1 & 1 & yes & 2 & yes \\
5 & higher preference for $P_{1}$ & 1 & 0.5 & yes & 2 & yes \\
6 & higher preference for $P_{2}$ & 0.5 & 1 & yes & 2 & yes \\
\hline
\end{tabular}

${ }^{a}$ In each of these cases, other parameters are given in Table 3.2.

${ }^{b}$ These parameter choices guarantee that $P_{1}$ would outcompete $P_{2}$ in the absence of the zooplankter. 
Table 3.2: Parameters in the diamond-shaped food web model

\begin{tabular}{||c|c|c|c||}
\hline Parameter & Description & Value & Units \\
\hline$N_{0}$ & influx water nutrient concentration & $(1,15)$ & $\mathrm{mmol} \mathrm{N}^{-3}$ \\
$D$ & mixing rate & $\left(10^{-4}, 10^{-1}\right)$ & $d^{-1}$ \\
$\mu_{1}$ & maximum growth rate of $P_{1}$ & 1.0 & $d^{-1}$ \\
$\mu_{2}$ & maximum growth rate of $P_{2}$ & 0.8 & $d^{-1}$ \\
$k_{1}$ & $P_{1}$ growth half-saturation constant & 0.1 & $\mathrm{mmol} \mathrm{Nm}^{-3}$ \\
$k_{2}$ & $P_{2}$ growth half-saturation constant & 0.15 & $\mathrm{mmol} \mathrm{Nm}^{-3}$ \\
$m_{p}$ & non-grazing phytoplankton mortality rate & 0.01 & $d^{-1}$ \\
$g_{\max }$ & maximum grazing rate & 2.0 & $d^{-1}$ \\
$k_{\text {sat }}$ & grazing half-saturation constant & 10 & $\mathrm{mmol} \mathrm{Nm}^{-3}$ \\
$\gamma$ & zooplankton growth efficiency & 0.7 & \\
$m_{z}$ & zooplankton mortality rate & 0.1 & $d^{-1}$ \\
\hline
\end{tabular}



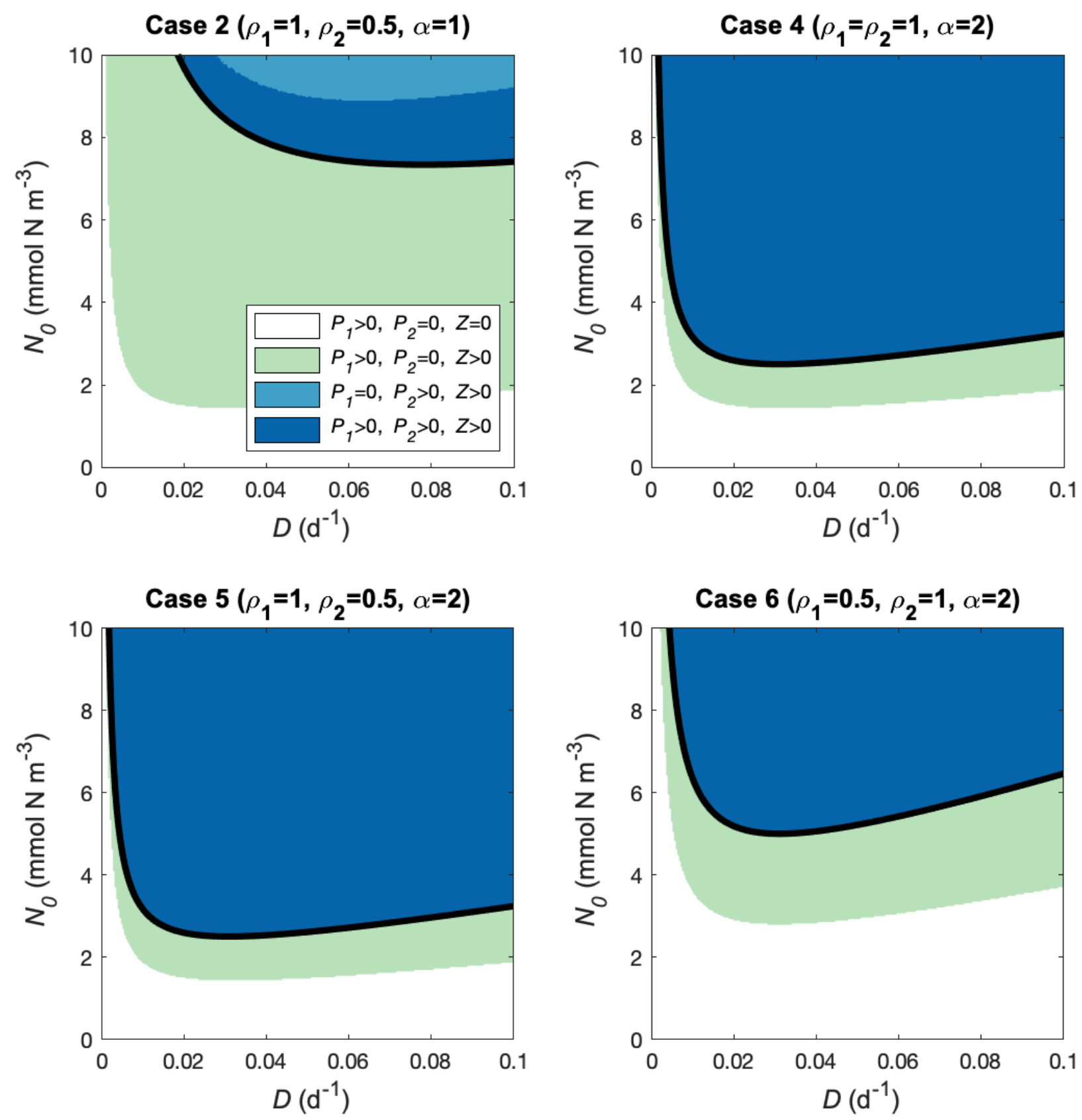

Figure 3-3: Asymptotic behavior of Cases 2 and 4-6 of model (3.2)-(3.4) as a function of $N_{0}$ and $D$. For each parameter combination we chose initial conditions at random from uniform distributions $\left(N(0) \sim U[0,15], P_{1}(0) \sim U[0,2], P_{2}(0) \sim U[0,2], Z(0) \sim U[0,10]\right)$, and simulated the model from $t=0$ to $t=10,000$ days. Colors indicate the asymptotic result. Along the black curve $r_{2}=0$ (cf., Eq 3.9); below the curve, $r_{2}<0$ and above the line $r_{2}>0$. A fifth outcome, in which $N=N_{0}$ and $P_{1}=P_{2}=Z=0$, is not illustrated in this graph. When $N_{0}<R_{1}^{*}$, this "washout equilibrium" is stable. The washout region is too small to be seen in the graphs above. 
$\alpha>1$ and at low densities of $P_{2}$ where switching provides a grazing refuge for an invading phytoplankton type (Fig. 3-4). Synergistic grazing is not unique to the KTW functional response and similar mathematical artifacts can be found in other functional responses (Gentleman et al., 2003).

In the KTW functional response, the zooplankton total ingestion rate depends on the total available phytoplankton biomass independent of the proportions of different phytoplankton types (Eq. 3.9). The ingestion rate of individual phytoplankton types, or the distribution of the total grazing pressure, depends on the proportion of a phytoplankton type in the environment and on the zooplankton preference. Consider the scenario in which we begin with $P_{1}>0$ and $P_{2}=0$ and calculate the grazing rate on $P_{1}$ as we introduce $P_{2}$ to the system. The total grazing rate must increase because the total phytoplankton biomass is increasing. However, if the grazer exhibits switching $(\alpha>1)$, then the distribution of grazing pressure will be heavily skewed towards $P_{1}$ since $P_{2}$ exists at low densities. In this case, the grazing rate on $P_{1}$ will increase as $P_{2}$ is introduced, even though the density of $P_{1}$ is being held constant. Perhaps counterintuitively, synergistic grazing has a stronger effect when the predator has a strong preference for the less abundant phytoplankton type because the value of $\rho_{i}$ acts as a modifier of the "effective biomass" in the system. If $\rho_{2}$ is large, then more effective biomass is added to the system when $P_{2}$ is introduced and the grazing rate changes faster than it would if the zooplankton had a lower preference for $P_{2}$. The phenomenon of synergistic grazing is related to an artifact of the functional response identified by Vallina et al. (2014), in which subdividing prey types into multiple identical species causes a change in the relative proportion of that prey type in the zooplankton's diet, even though the combined amount of that prey type remains the same.

Synergistic grazing alters the dynamics of the diamond-shaped food web model in interesting ways. It has a larger effect when switching behavior is stronger and when the preference for the weaker phytoplankton type is larger (Fig. 3-4). In some cases, the synergistic grazing produced by switching can result in coexistence criteria that are biologically improbable. For example, in a model with no switching, the invasion growth rate of $P_{2}$ will decrease as the zooplankton preference for $P_{2}$ increases (Eq. 3.9). However, when switching is included, the invasion growth rate is independent of $\rho_{2}$, meaning that $P_{2}$ will be able to invade the system no matter how large the grazer preference for $P_{2}$ becomes. This occurs because switching creates a grazing refuge for phytoplankton at low densities. Grazer preference has no effect when a phytoplankton type is rare because all of the 


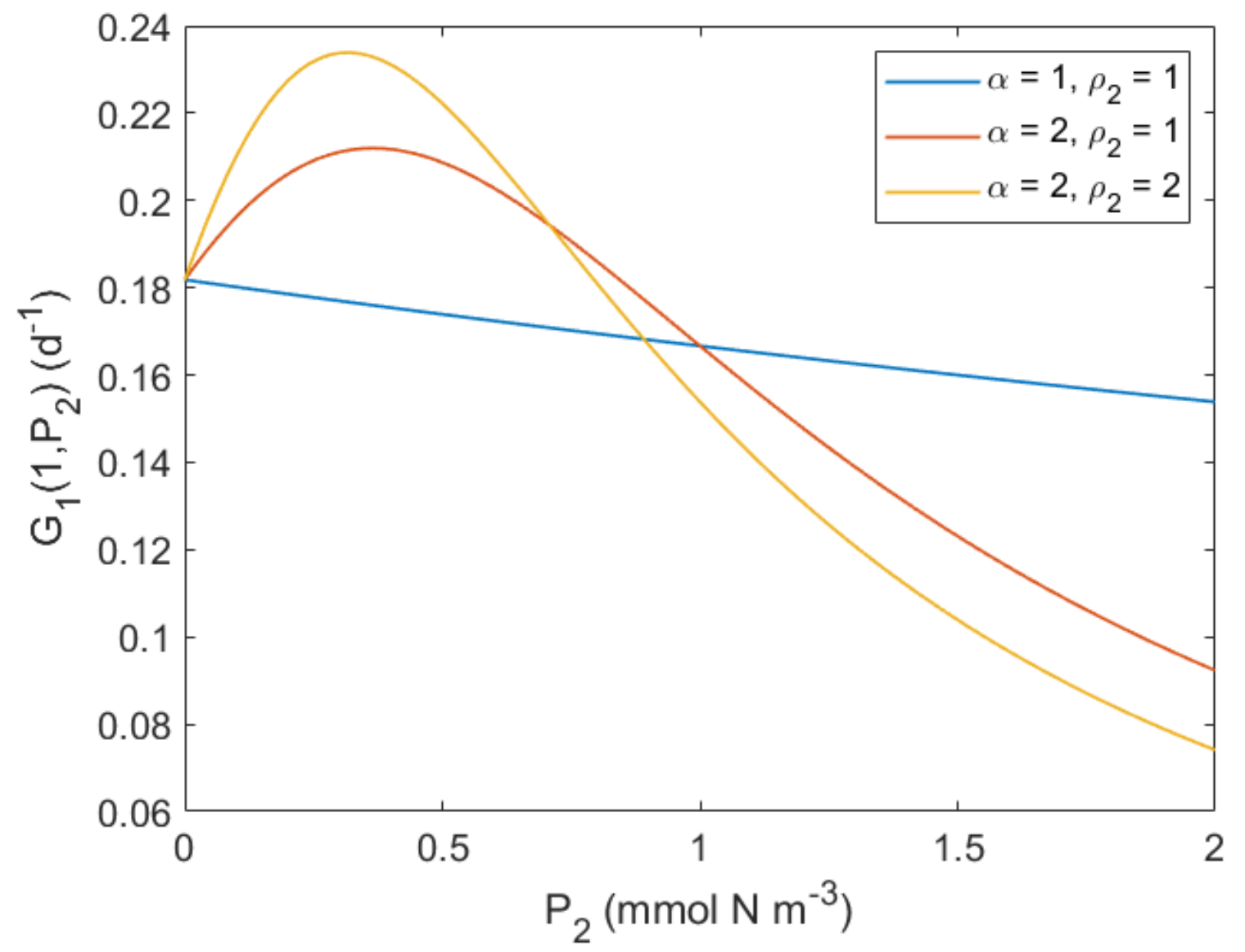

Figure 3-4: $G_{1}\left(1, P_{2}\right)$ is the grazing rate on $P_{1}$ as a function of the density of $P_{2}$, when $P_{1}=1$. In these graphs, synergistic grazing occurs when $\partial G_{1}\left(1, P_{2}\right) / \partial P_{2}>0$; i.e., when the zooplankter exhibits switching behavior $(\alpha=2)$. Other parameters are given in Table 3.2. 
grazing is focused on the more common phytoplankton type.

\subsection{Discussion}

Predator switching behavior is frequently observed in laboratory and field studies (Hughes and Croy, 1993; Kempf et al., 2008; Kiørboe et al., 1996; Murdoch, 1969; Murdoch et al., 1975); modeling work has shown that it stabilizes dynamics and increases diversity (Armstrong, 1999; Morozov, 2010; Prowe et al., 2012; Vallina et al., 2014a). Historically, modelers have used simple phenomenological functional responses to represent the complex phenomenon of switching (Gentleman et al., 2003). The KTW functional response was created in response to the existence of certain mathematical artifacts of these functions, particularly antagonistic grazing (Vallina et al., 2014b). Here, we have evaluated the dynamics of the KTW functional response in a diamond-shaped food web model with respect to coexistence between competing phytoplankton mediated by grazer preference and switching. The purpose of these analyses was to, first, confirm that this novel functional response formulation is consistent with classical results concerning grazer mediated coexistence, and second, to provide context for larger-scale results for the effect of distributed grazing on phytoplankton diversity in more complex, size-structured models.

We found that, with the KTW functional response, coexistence was possible via either grazer preference or switching alone. Switching was generally the more powerful mechanism for generating coexistence, allowing for coexistence between competing phytoplankton types across a broad set of environmental conditions. In contrast, grazer preference in the absence of switching must be carefully balanced with the competitive ability of the phytoplankton types to allow for coexistence. These results are consistent with past work showing that predation can reverse competitive exclusion, but only if model parameters are precisely balanced (Cramer and May, 1972; May, 1973; Slobodkin, 1980). Switching, however, has been shown to increase phytoplankton diversity on a global scale (Vallina et al., 2014b). Typically, larger phytoplankton size classes, which tend to be weaker competitors for nutrients, are added sequentially as the total phytoplankton biomass of the system increases (Armstrong, 1994; Vallina et al., 2014a; Ward et al., 2014). We calculated the criteria under which a weaker phytoplankton competitor can invade the stable equilibrium of a stronger phytoplankton competitor and a grazer, finding that similar to the results referenced 
above, if switching is included, a weaker phytoplankton type can coexist with a stronger type as long as the the nutrient supply is sufficiently high.

The interaction between zooplankton preference and switching in the KTW parameterization can produce some unexpected behaviors. Synergistic grazing is an example of a potentially problematic characteristic that emerges as a result of the formulation of switching in Eq 3.1. The effects of synergistic grazing are most evident when switching is strong and when zooplankton have a preference for the weaker competitor. Synergistic grazing arises directly from the fact that the total grazing rate and the distribution of grazing onto individual phytoplankton types are calculated as independent terms. This functional form was chosen specifically to avoid the problem of antagonistic grazing (Vallina et al., 2014b), another problematic characteristic of distributed-grazing functional responses. There appears to be a trade-off among functional responses that include switching, since all such responses of which we are aware include either antagonistic grazing or synergistic grazing. Indeed, Teramoto et al. (1979) showed that within a class of switching models, those that preclude synergistic grazing must exhibit antagonistic grazing. It remains unclear whether the result can be generalized to a broader class of models (e.g., models that include preferences). It also is unknown whether the reverse is true: i.e., that a switching functional response that does not exhibit antagonistic grazing must exhibit synergistic grazing.

In general, synergistic grazing is likely a smaller problem for modelers than antagonistic grazing. The effect of synergistic grazing only occurs at low phytoplankton concentrations and its effect is small except for certain parameterizations (strong switching and zooplankton preference for the weaker competitor). Antagonistic grazing, on the other hand, becomes increasingly problematic for models with larger numbers of phytoplankton types or size classes. For this reason, the KTW function represents a significant improvement over previous functional responses. The existence of synergistic grazing should not be taken as an argument against its use, as long as it is applied thoughtfully and with awareness of its behavior.

The diamond-shaped food web model has, historically, been used to describe the growth of microbial species in a chemostat and how grazing on one or more competitors can permit coexistence (Fredrickson, 1977). The true diamond-shaped model represents grazing on both competing species (e.g. Jost et al., 1973). A closely related class of models includes only grazing on one of the competing species, usually the stronger competitor (Butler and Wolkowicz, 1986). Analysis of 
these chemostat models has resulted in a few key ecological principles concerning the outcome of competition between two species. First, pure competition for a single resource results in the survival of at most a single competitor (Butler and Wolkowicz, 1985). With the inclusion of grazing on one or both of the competitors, the models show a progression of stable equilibrium points with increasing steady-state nutrient concentration - nutrient substrate only, persistence of the stronger competitor, persistence of the stronger competitor and the grazer, and finally persistence of both competitors with the grazer (Butler and Wolkowicz, 1986). Here, I have modified the classical diamond-shaped food web model by using the KTW functional response, which includes both grazer preference and switching as independent mechanisms. Previous studies of the diamond-shaped food web model have examined only grazer preference as a way of promoting coexistence. The fact that the model using the KTW function behaves similarly to models using Lotka-Volterra or Monod-like grazing functional responses adds robustness to the results. Additionally, I have found that switching is in fact a much stronger mechanism for promoting coexistence between competitors. Moving forward, this work encourages modelers to consider multiple aspects of zooplankton grazing and how they may have different effects of the phytoplankton community.

Switching is an interesting ecological phenomenon since the functional responses used to model it could represent multiple ecological mechanisms that all have the same emergent behavior: frequencydependent differential grazing on multiple available phytoplankton types. The classical view of switching is that it arises from zooplankton behavior such as changes in feeding strategy or time budget optimization in response to a variable phytoplankton community (Mariani and Visser, 2010; Paffenhöfer, 1984; Strom et al., 2000). Alternatively, the zooplankton represented in this model could be viewed as a generalized micro-grazer community rather than a single species. Under this scenario, the switching "behaviors" represent internal changes in the composition of the zooplankton community as the composition of the phytoplankton community evolves through time (Fasham et al., 1990). When one phytoplankton type becomes very abundant, specialized predators of that type also become more abundant and the integrated grazer community becomes more efficient at grazing that phytoplankton type. Conversely, when a phytoplankton type is rare, its specialized predators will also be rare and the proportion of total grazing pressure on this phytoplankton type will be small. From either perspective, the mathematical consequences are the same and so the parameterizations used here, including the KTW functional response, could be used to represent 
either ecological scenario. There is, however, an implicit assumption in using switching to represent community-scale changes in the zooplankton. Namely, the functional response assumes that the composition of the zooplankton community changes instantaneously following the abundance of phytoplankton in the environment. In this study, we have formulated our model to generally represent microzooplankton grazers, which typically have growth rates similar to their phytoplankton prey. The assumption would be less appropriate when considering mesozooplankton grazers that have significant generation times and seasonal-scale life cycles. 


\section{Chapter 4}

\section{Increased nutrient supply and}

\section{zooplankton switching increase diversity in a size-structured NPZ model}

\subsection{Introduction}

In the Chapter 3, I explored the dynamics of the "Kill-the-Winner" (KTW) functional response (Vallina et al., 2014b) and demonstrated that zooplankton switching allows for coexistence between two competing phytoplankton types under circumstances that otherwise (i.e., without switching) would result in competitive exclusion of the weaker phytoplankton type. This result was shown for a diamond-shaped food web model that included only two phytoplankton types. This simple model provides a mechanistic context for work by Vallina et al. (2014), which has shown that zooplankton switching increases phytoplankton diversity at a global scale and that higher diversity results in increased primary productivity. Other grazing functional responses have been shown to promote coexistence and increase diversity as well (Armstrong, 1999; Morozov, 2010; Prowe et al., 2012). In general, grazing promotes coexistence by increasing mortality on the "winning" phytoplankton type thereby relieving the competitive pressure on competitively weaker phytoplankton types. This can be achieved either by specific grazers with different grazing rates, or via generalist grazers that exhibit switching. 
Looking forward to the final chapter of this thesis, I am interested in describing patterns in phytoplankton community size structure and understanding the physical and biological conditions that lead to those patterns. Real phytoplankton communities, however, are unlikely to consist of only two phytoplankton types. So while the diamond-shaped food web model from the previous chapter is useful, in a theoretical sense, for the purposes of examining fundamental ecological forces that shape communities on the scale of competition between individual phytoplankton types, a more complex model is needed for improving our understanding of the dynamics of real-world plankton ecosystems.

In many NPZ models, the phytoplankton are structured by size (e.g. Armstrong, 1999; Baird and Suthers, 2007; Gin et al., 1998; Moloney and Fields, 1991; Poulin and Franks, 2010; Ward et al., 2014; Zhou, 2006). This class of NPZ models is built upon an understanding of the ways that cell size is systematically related to many important metabolic rates and biogeochemical characteristics (Chisholm, 1992). They typically include one or more allometric scaling laws, which mathematically describe the relationships between cell size and model parameters (Verdy et al., 2009). These allometric relationships reduce the number of parameters in the model and make the construction and analysis of size-structured models more feasible, since each size class does not need to be parameterized independently.

Theoreticians have leveraged allometric scalings to investigate the effect on phytoplankton diversity of bottom-up and top-down controls on phytoplankton communities. The typical pattern observed in these models is that larger phytoplankton size classes are added to the system sequentially as nutrients are made more available, since larger phytoplankton have higher nutrient requirements (Armstrong, 1994; Poulin and Franks, 2010; Ward et al., 2014). However, this increase in diversity can only occur if sufficiently strong top-down pressure from zooplankton grazing is present; otherwise the smallest phytoplankton size class tends to dominate the community (Poulin and Franks, 2010).

In this chapter, I extend the diamond-shaped food web model into a size-structured NPZ model that includes an arbitrary number of phytoplankton size classes. This model includes the KTW functional response to represent zooplankton switching. Analysis of the size-structured model illustrates how the dynamics described in the previous chapter play out in a larger community, and what the consequences are for phytoplankton communities structured by size. Specifically, I investigate 
how nutrient supply and zooplankton grazing (including switching) interact to promote increased numbers of coexisting size classes and greater evenness of the biomass distribution across coexisting size classes.

The primary purpose of extending the diamond-shaped food web model into a size-structured model, as presented here, is to investigate how the competitive relationships between individual pairs of phytoplankton types play out in a community composed of many types. This iterative approach of increasing model complexity allows me to maintain a mechanistic understanding of the ecological processes that lead to patterns of diversity observed in more complex models (e.g., Vallina et al., 2014a). Of particular interest is the specific effect of zooplankton switching and whether the effect of switching as described by the KTW functional response differs from its effect when described by other, previously studied, kinds of distributed grazing (Armstrong, 1999). Secondarily, the analysis of this particular size-structured model using the KTW functional response confirms that the model behaves similarly to previous size-structured models using different grazing functional responses (Armstrong, 1994). As such, I expect that the model below will display the characteristic behavior of this class of models, in which phytoplankton size classes are introduced in order of increasing size as the environmental nutrient concentration is increased (Armstrong, 1994; Ward et al., 2014).

\subsection{Model Construction and Simulation}

Phytoplankton communities are commonly composed of many different types coexisting simultaneously. Size is a particularly important trait for structuring these communities since cell size is related to many important characteristics of phytoplankton, including growth rates and nutrient affinities (Chisholm, 1992). To explore the effects of zooplankton switching on phytoplankton communities structured by cell size, we extended the diamond-shaped model from the previous chapter to an NPZ model that includes an arbitrary number of phytoplankton size classes (Fig. 4-1). This model contains a single nutrient resource $\left(N_{0}\right)$ and $n$ phytoplankton size classes $\left(P_{i}\right)$. A single zooplankton type $(Z)$ distributes grazing across all phytoplankton size classes following KTW. The 
size-structured model is written as

$$
\begin{aligned}
\frac{d N}{d t} & =D\left(N_{0}-N\right)-\sum_{i=1}^{n} \mu_{i} P_{i} \frac{N}{k_{i}+N} \\
\frac{d P_{i}}{d t} & =\mu_{i} P_{i} \frac{N}{k_{i}+N}-G_{i}\left(P_{1}, \ldots, P_{n}\right) Z-\left(m_{p}+D\right) P_{i} \\
\frac{d Z}{d t} & =\gamma \sum_{i=1}^{n} G_{i}\left(P_{1}, \ldots, P_{n}\right) Z-\left(m_{z}+D\right) Z
\end{aligned}
$$

with grazing function,

$$
G_{i}\left(P_{1}, P_{2}, \ldots, P_{n}\right)=g_{\max } \frac{\rho_{i} P_{i}^{\alpha}}{\sum_{j=1}^{n} \rho_{j} P_{j}^{\alpha}} \frac{\sum_{j=1}^{n} \rho_{j} P_{j}}{k_{s a t}+\sum_{j=1}^{n} \rho_{j} P_{j}}
$$

Size classes are defined such that the volume of each size class is logarithmically-spaced from $10^{-1}$ to $10^{3} \mu m^{3}$ (Table 4.1 ). The parameters $\mu_{i}$ and $k_{i}$ are the growth rate and nutrient affinity for each phytoplankton size class. These parameters were scaled allometrically such that the smallest phytoplankton size classes have the largest $\mu$ and the smallest $k$ following previous size-structured phytoplankton models (Verdy et al., 2009; Ward et al., 2014). The $R^{*}$ values for each phytoplankton size class are consequently ordered such that $R_{1}^{*}<R_{2}^{*}<\ldots<R_{n}^{*}$. In the absence of any grazing, the smallest size class will out-compete all other size classes (Tilman, 1977). Other parameters used to simulate the model are similar to those selected for the diamond-shaped model (Table 4.1).

We simulated the model over a range of nutrient input values $\left(N_{0}\right)$ under both no-switching $(\alpha=1)$ and switching $(\alpha>1)$ scenarios (Table 4.1). We also ran simulations over a range of values of $k_{\text {sat }}$ and $\alpha$ to test the effects of increased zooplankton grazing strength and the strength of switching on phytoplankton diversity (Table 4.1). Diversity in the model was evaluated using the distribution of biomass between size classes as a proxy. Richness $(\mathrm{R})$ was defined as the number of size classes coexisting at equilibrium. A given size class was counted if it constituted at least $1 \%$ of the total phytoplankton community biomass. Evenness (E) was defined as

$$
E=\frac{-\sum_{i=1}^{R} p_{i} \ln p_{i}}{\ln R}
$$

where $p_{i}$ is the biomass proportion of size class $i$ in the community (Pielou, 1966). Evenness 


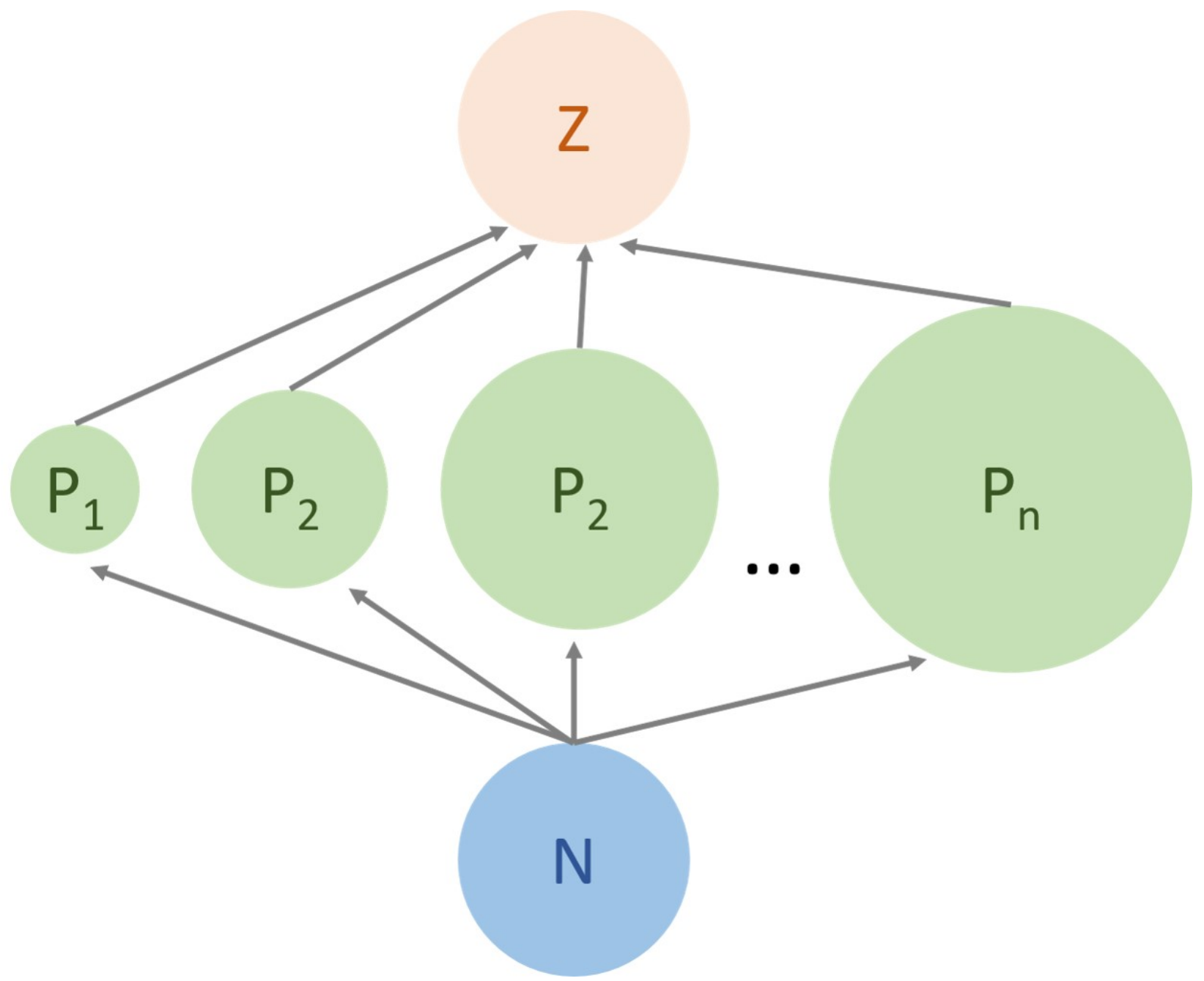

Figure 4-1: Size-structured model configuration with one nutrient $(\mathrm{N})$ box, $n$ phytoplankton boxes $(\mathrm{P})$, and one zooplankton box $(\mathrm{Z})$. 
Table 4.1: Parameter values used to simulate the size-structured phytoplankton community model

\begin{tabular}{||c|c|c|c||}
\hline Parameter & Description & Value & Units \\
\hline$N_{0}$ & influx water nutrient concentration & $(0,15)$ & $m m o l ~ N m^{-3}$ \\
$D$ & mixing rate & 0.05 & $d^{-1}$ \\
$n$ & number of phytoplankton size classes & 10 & $\mu m^{3}$ \\
$x$ & cell volume & $10^{\left[-1+\frac{4}{n-1}(i-1)\right]}, i=1: n$ & $d^{-1}$ \\
$\mu$ & maximum phytoplankton growth rate & $0.5 x^{-0.24}$ & $m m^{-3}$ \\
$k$ & nutrient uptake half-saturation constant & $0.14 x^{0.33}$ & $d^{-1}$ \\
$m_{p}$ & non-grazing phytoplankton mortality rate & 0.01 & $d^{-1}$ \\
$g_{\text {max }}$ & maximum grazing rate & 2.0 & $m m o l ~ N m^{-3}$ \\
$k_{\text {sat }}$ & grazing half-saturation constant & $(10,20)$ & $d^{-1}$ \\
$\gamma$ & zooplankton growth efficiency & 0.7 & \\
$m_{z}$ & zooplankton mortality rate & 0.1 & \\
$\rho_{i}$ & zooplankton preference & $(1,5)$ & \\
$\alpha$ & zooplankton switching strength & &
\end{tabular}


ranges from 0 to 1 , with higher values indicating a more even distribution of biomass between phytoplankton size classes. The Shannon Diversity Index (H, sometimes called Shannon Entropy) incorporates both size class richness and biomass evenness (Shannon, 1948).

$$
H=-\sum_{i=1}^{R} p_{i} \ln p_{i}
$$

The model includes uniformly distributed zooplankton preference parameters $\left(\rho_{i}\right)$, therefore ignoring the effect that preference has on phytoplankton diversity (Table 4.1). This decision was made because achieving coexistence through preference alone in the size-structured model is quite difficult. In the analysis of the diamond-shaped food web model in the previous chapter, zooplankton preference was included as a mechanism separate from switching that was capable of promoting coexistence between two competing phytoplankton types. In the absence of switching, however, the preference parameters must be carefully balanced in order to achieve coexistence, resulting in relatively narrow regions of the parameter space where coexistence is possible. This problem is more acute when the number of phytoplankton size classes is large. Additional assumptions need to be made concerning the distribution of preference values across the phytoplankton size classes (e.g., linearly vs. logarithmically). In my experience simulating this model, I found that as the number of size classes was increased, it became increasingly difficult to balance zooplankton preference against the differences in phytoplankton competitive ability and achieve coexistence. I have chosen to simplify our analysis for the size-structured model and use a constant zooplankton preference across all phytoplankton size classes.

\subsection{Results}

Total phytoplankton biomass (summed over all size classes) and zooplankton biomass at equilibrium depends on the incoming nutrient concentration $\left(N_{0}\right)$, but does not have any dependence on the strength of zooplankton switching (Fig. 4-2). Equilibrium phytoplankton biomass increases with $N_{0}$ up to the threshold value where zooplankton can survive, after which increasing $N_{0}$ results in a static phytoplankton biomass at equilibrium and increased zooplankton biomass. The independence between total phytoplankton biomass and zooplankton switching arises from the formulation of 
KTW, since the total grazing rate depends only on the total phytoplankton biomass and not on the distribution of biomass between size classes (Eq. 4.4).

Under the no-switching scenario $(\alpha=1)$, increased nutrient input does not increase size class richness (Fig. 4-2). For all values of $N_{0}$, only the smallest phytoplankton size class existed at equilibrium (Fig. 4-3a). In simulations where zooplankton did exhibit switching $(\alpha>1)$, increasing $N_{0}$ resulted in increased richness (Fig. 4-2). Stronger switching (higher values of $\alpha$ ) results in more coexisting size classes at equilibrium (Fig. 4-2). Stronger switching also results in a more even distribution of biomass between size classes (Fig. 4-3).

Phytoplankton size classes were introduced in order of increasing size such that, for any given set of $j$ coexisting size classes, only the smallest $j$ size classes were present at equilibrium (Fig. 43). The threshold value of $N_{0}$ at which the next largest size class can invade the system can be predicted by extending Equation 3.10 to a model with $n$ phytoplankton types ordered in size. To do so, consider the equilibrium at which all size classes smaller than the $\mathrm{j}$-th size class are coexisting, and all larger size classes are extinct. I defined the quantity $P_{j}^{*}$ as the vector containing the values of $P_{i}$ at this equilibrium, with $P_{i}>0$ for $i<j$ and $P_{i}=0$ for $i \geq j$. Similarly, $N_{j}^{*}$ and $Z_{j}^{*}$ are the equilibrium values of $N$ and $Z$. Then the invasion growth rate of $P_{j}\left(r_{j}\right)$ at the equilibrium point $\left(N_{j}^{*}, P_{j}^{*}, Z_{j}^{*}\right)$ is given by,

$$
r_{j}=\left.\frac{1}{P_{j}} \frac{d P_{j}}{d t}\right|_{\left(N_{j}^{*}, P_{j}^{*}, Z_{j}^{*}\right)}= \begin{cases}\frac{\mu_{j} N_{j}^{*}}{k_{j}+N_{j}^{*}}-m_{P}-D-\frac{\rho_{j} g_{\max } Z_{j}^{*}}{k_{s a t}+\rho_{j} P_{j}^{*}} & \text { if } \alpha=1 \\ \frac{\mu_{j} N_{j}^{*}}{k_{j}+N_{j}^{*}}-m_{P}-D & \text { if } \alpha>1 .\end{cases}
$$

For $r_{j}>0$, the j-th phytoplankton size class can invade the system (Fig. 4-4-4-5).

Highest diversity in the model occurred under coincident high nutrient input and strong switching (Fig. 4-4). Similarly, we observed high diversity when grazing pressure, as measured by $k_{\text {sat }}$, was increased alongside $N_{0}$, provided that $\alpha>1$ (Fig. 4-4). No single factor was sufficient to support phytoplankton diversity in the size-structured model. The recipe for high diversity, in this model, includes sufficiently high nutrient supply, sufficiently strong grazing pressure, and some degree of zooplankton switching. 

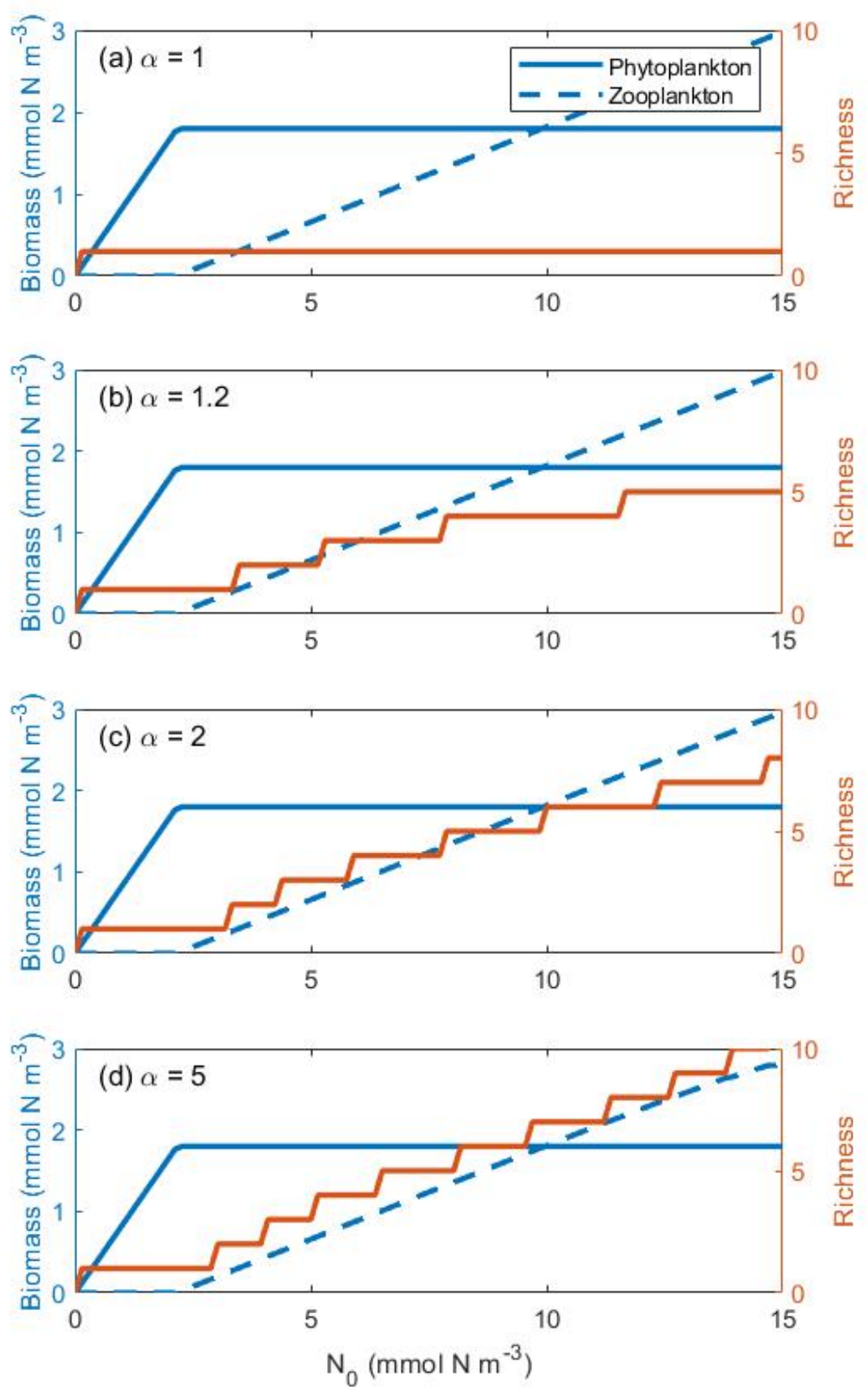

Figure 4-2: Total phytoplankton community biomass and zooplankton biomass at equilibrium, as well as phytoplankton size class richness, as a function of input nutrient concentration $\left(N_{0}\right)$ under both (a) non-switching and (b-d) switching scenarios. 
(a) $\alpha=1$

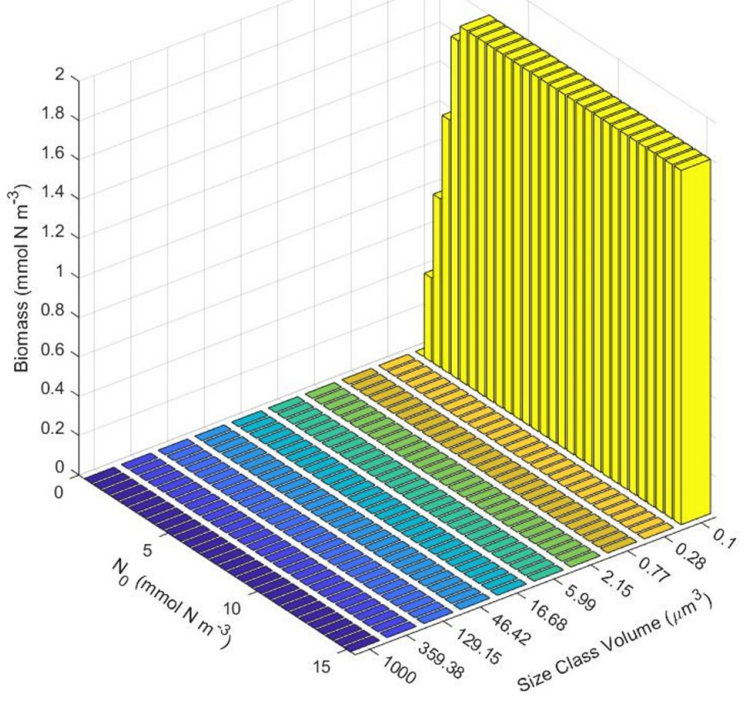

(c) $\alpha=2$

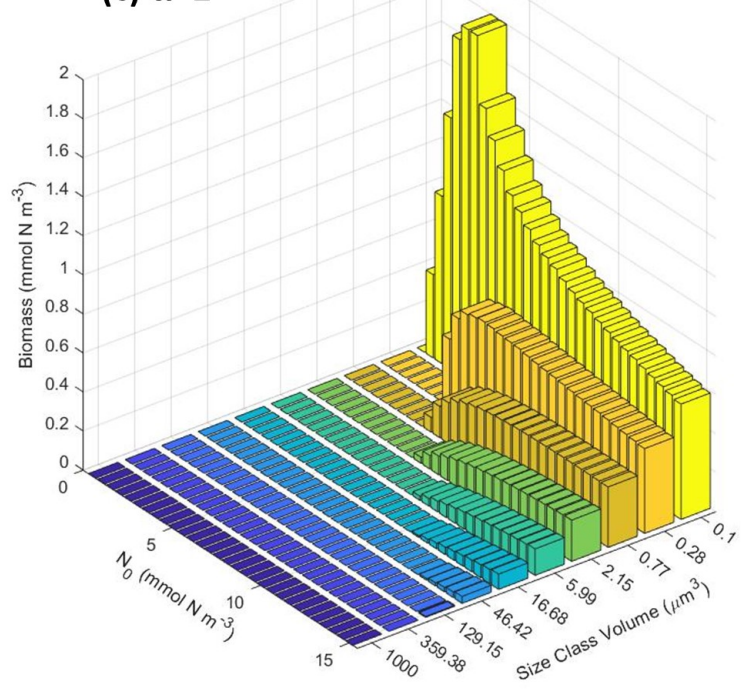

(b) $\alpha=1.2$

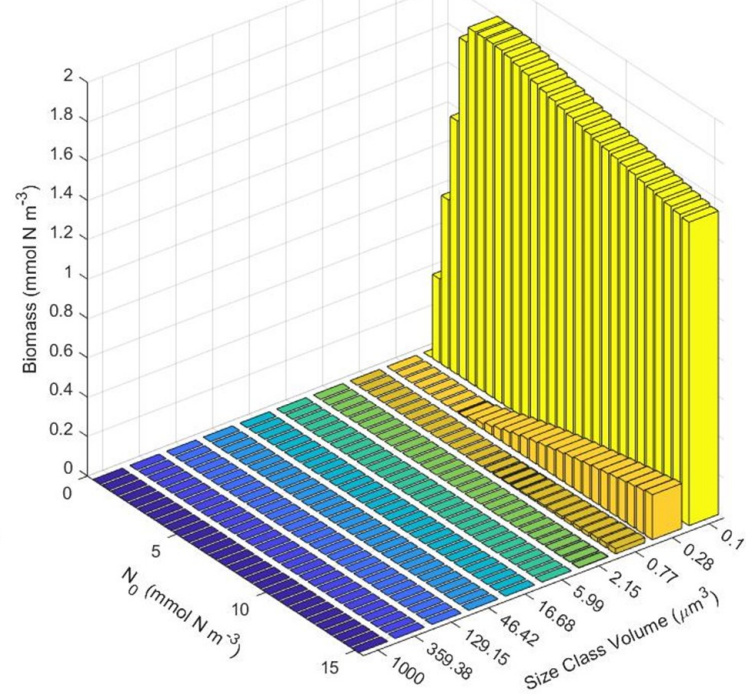

(d) $\alpha=5$

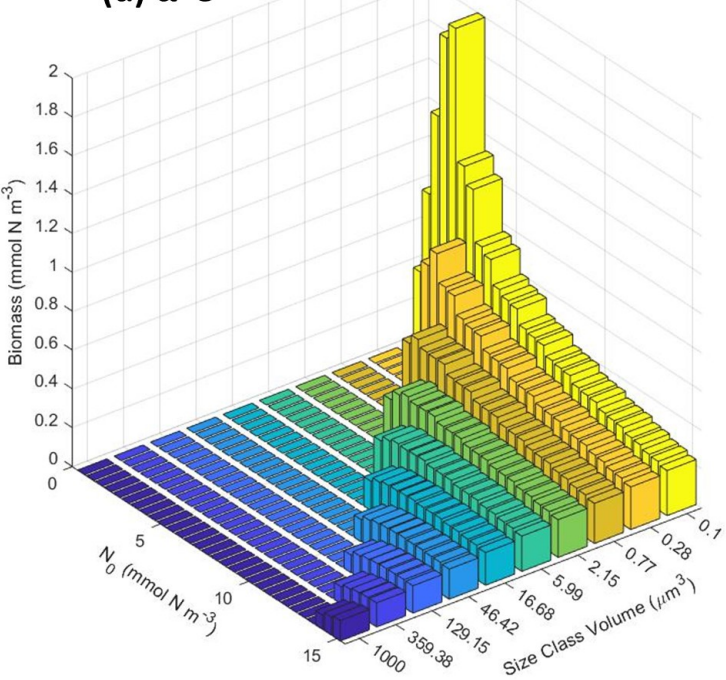

Figure 4-3: Distribution of biomass between the 10 phytoplankton size classes at equilibrium for different values of $\alpha$ as a function of $N_{0}$. 

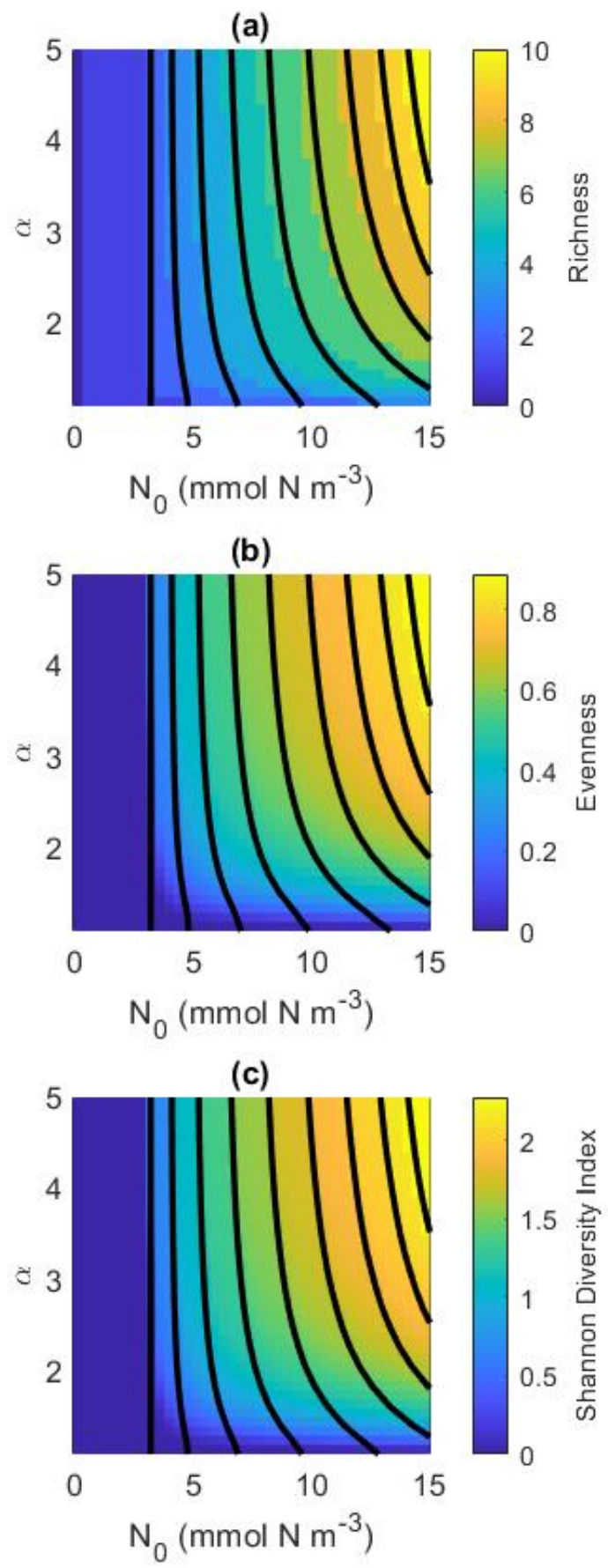

Figure 4-4: Phytoplankton community size class (a) richness, (b) evenness, and (c) Shannon Diversity as a function of $N_{0}$ and $\alpha$. The contours show $r_{j}=0$ for each size classes $j=1: 10$. For these simulations, $k_{\text {sat }}=15$. 

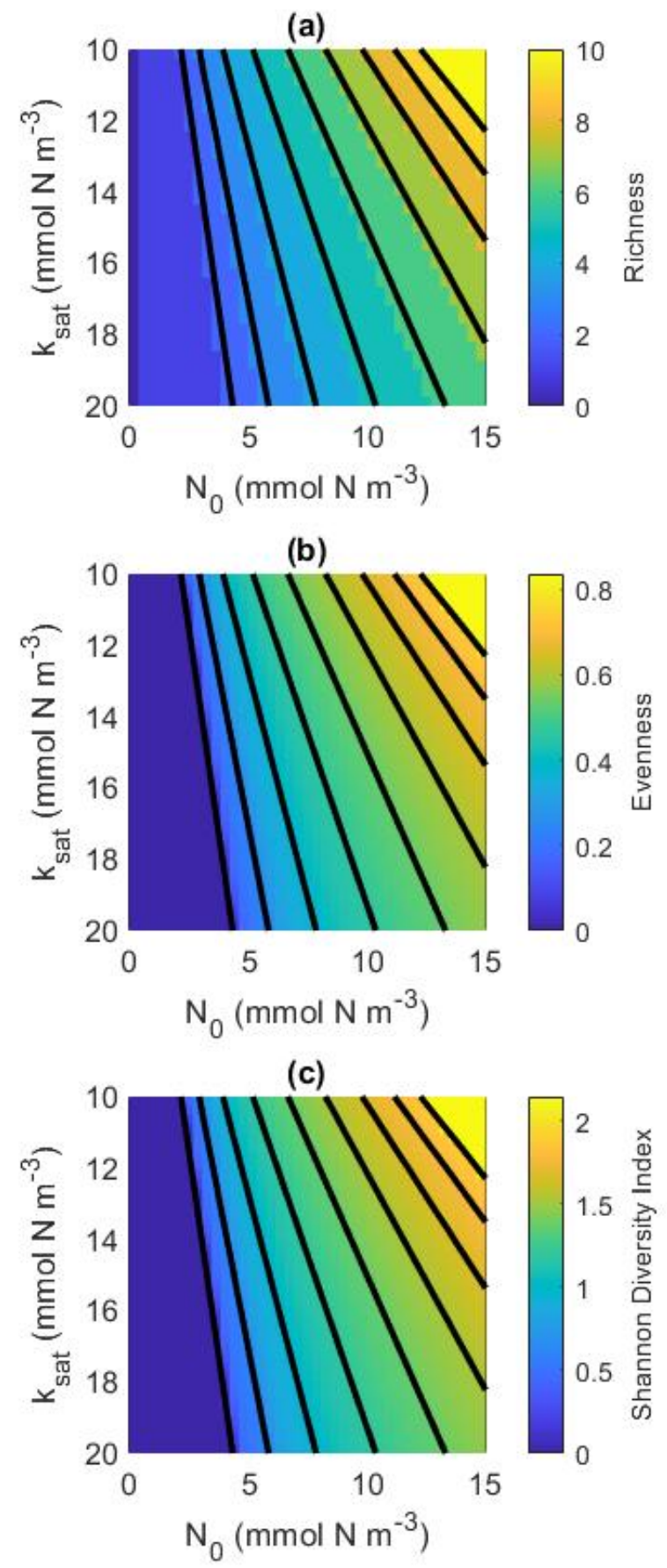

Figure 4-5: Phytoplankton community size class (a) richness, (b) evenness, and (c) Shannon Diversity as a function of $N_{0}$ and $k_{s a t}$. The contours show $r_{j}=0$ for each size classes $j=1: 10$. The vertical axis is plotted in the reverse direction because smaller values of $k_{\text {sat }}$ correspond to stronger zooplankton grazing pressure. For these simulations, $\alpha=2$. 


\subsection{Discussion}

Analysis of the size-structured NPZ model indicates that increased nutrient supply alone has no effect on phytoplankton community size diversity (Fig. 4-2). The $\mathrm{R}^{*}$ theory of limiting nutrients for phytoplankton communities proposed by Tilman et al. (1977) asserts that the strongest phytoplankton competitor will reduce the environmental nutrient concentration below the level at which its competitors can survive, regardless of the incoming nutrient supply. Without switching, there is no equalizing mechanism to offset the competitive advantage of the smallest phytoplankton size class. As a result, increasing $N_{0}$ in the model results in no additional diversity; all increased productivity is passed up the $N \rightarrow P_{1} \rightarrow Z$ food chain. Switching, however, focuses grazing pressure onto whichever phytoplankton size class is most common in the community. Under the switching scenario, increasing $N_{0}$ results in higher diversity within the phytoplankton community, in terms of both size class richness and evenness.

These results indicate that both nutrient supply and zooplankton grazing play a role in determining phytoplankton diversity. Bottom-up and top-down controls work synergistically. Without any zooplankton switching to promote coexistence, an increase in nutrients does not increase diversity since increased productivity contributes only to the strongest phytoplankton competitor. Similarly, strong zooplankton switching on its own is not sufficient to promote high diversity since no amount of grazing pressure can make up for the fact that larger phytoplankton size classes require some minimum nutrient input to survive. It is only through the simultaneous action of both high nutrient input and strong grazing with switching that phytoplankton communities with high diversity are maintained (Figs. 4-3,4-4). As Poulin and Franks (2010) put it, "resources determine which classes can exist; predation determines which classes do exist." These results are consistent with recent work that examined both shipboard observations from Atlantic meridional transects, as well as simulations of a global biogeochemical model (Dutkiewicz et al., 2020). They found that the supply rate of limiting nutrients and size-selective grazing were important drivers of phytoplankton size-class diversity.

My analysis confirms that the use of of the KTW functional response does not fundamentally change the dynamics of comparable size-structured NPZ models studied by others (e.g., Armstrong, 1994; Poulin and Franks, 2010; Ward et al., 2014). The parameterization of zooplankton switch- 
ing in the KTW results in the same relationship between size-class diversity and environmental nutrient concentration established earlier using alternative representations of distributed grazing. Importantly, the parameter $\alpha$, which describes the strength of switching, plays an important role in modulating these patterns. Stronger switching results in greater size class diversity at the same level of nutrient input. It remains unclear, however, in what situations different switching strengths are most reasonable for describing the system, highlighting the need for additional research to fully understand the effects of zooplankton switching in real ecosystems. By focusing on the role of switching, my study compliments previous work by expanding the range of grazing behaviors described in size-structured models. Smith et al. 2016 had previously shown that the strength of zooplankton switching can increase phytoplankton diversity using the KTW functional response. My study contributes to a growing body of work that encourages both modelers and empiricists to think critically about how grazing is parameterized and what effects specific grazing behaviors may have on phytoplankton diversity.

Diversity in the model was sensitive to the strength of zooplankton switching (Fig. 4-4). Care is warranted when parameterizing the KTW functional response, since the value of $\alpha$ has such important implications for the simulated phytoplankton dynamics. Unfortunately, zooplankton switching is not something that is typically characterized by empiricists. Measuring $\alpha$ in a real ecosystem is certainly not a trivial task, since the parameter describes the emergent behavior of the zooplankton community arising from the integrated result of many individual behavioral changes (Kiørboe et al., 1996). Nevertheless, characterizing the variability in $\alpha$ in zooplankton communities is important. Even though $\alpha$ is difficult or impossible to directly measure in an ecosystem, a series of carefully designed prey-selectivity experiments (similar to those conducted by Hassell (1978)) could provide first-order estimates of the magnitude of zooplankton switching under certain conditions or specific phytoplankton assemblages.

The model presented here considers only nutrient limitation as a bottom-up control on phytoplankton growth, but light may also be an important control on phytoplankton communities. Phytoplankton cells of different sizes exhibit advantages under different light regimes, with smaller cells typically benefiting under light-limiting conditions (Finkel, 2001; Geider et al., 1986) and larger cells showing reduced susceptibility to photoinhibition under high-light conditions (Key et al., 2010). Light absorption of phytoplankton cells is a function of the optical cross-section, which depends on 
both cells size and pigment concentration (Morel and Bricaud, 1981). The specific absorption of light decreases with cell size due to what is referred to as the "package effect", in which high concentrations of light-absorbing pigments self-shade and reduce the effective cross-sectional area compared to the same amount of pigment divided into many, smaller packets (Morel and Bricaud, 1981). This self-shading, however, is exactly the mechanism that makes larger cells less susceptible to photoinhibition (Key et al., 2010). Therefore, there exists a size-based trade-off between phytoplankton competitors under different light regimes. These relationships would modulate the patterns in phytoplankton diversity presented here, based on the the limitation (or co-limitation) of light alongside nutrients.

In the following chapter, I will be examining the patterns in phytoplankton community size structure within the shelfbreak front region of the Northeast U.S. Shelf. Based on the analysis presented here, I expect to find increased diversity (where diversity is represented by the evenness of the distribution of biomass between size classes) where upwelling supplies increased nutrients to the euphotic zone and where zooplankton grazing is simultaneously strong. The shelfbreak jet associated with the front generates persistent upwelling at the front (Zhang et al., 2011). And while there is a lack of data describing the distribution of zooplankton grazing pressure in the region, it is reasonable to assume that strong zooplankton grazing would be found in areas of increased primary productivity. Therefore, I am proposing the following hypothesis for patterns in phytoplankton community size structure on the Northeast U.S. shelf. Frontal upwelling generates high primary productivity at the shelfbreak front. Strong grazing, accompanied by some form of zooplankton switching, is also found at the shelfbreak front. The combination of these two factors results in higher phytoplankton diversity at the front compared to water masses either onshore or offshore of the front. We also expect that the signal of increased diversity at the front will be stronger during the spring season than in the summer, when stratification may lead to depletion of nutrients in surface waters. 


\section{Chapter 5}

\section{Patterns in phytoplankton community size structure in the shelfbreak front region of the Northeast U.S. Shelf}

\subsection{Introduction}

The Northeast U.S. Shelf region supports a productive marine ecosystem. One of the key hydrographic features of this region is the shelfbreak front, a sharp density gradient separating the colder and fresher water on the shelf from the warmer and saltier water on the slope. Studies of both satellite and in situ observations have shown local enhancement of chlorophyll at the front (Marra et al., 1982; O'Reilly and Busch, 1984; Ryan et al., 1999). It has been proposed that high productivity at the front is supported by upwelling that results from divergence of surface water on the inshore side of the front (Zhang et al., 2011), convergence of the bottom boundary layer (Linder et al., 2004), or vertical motion associated with frontal meanders (Zhang and Gawarkiewicz, 2015). Interestingly, however, despite the persistent upwelling generated by the shelfbreak jet, chlorophyll enhancement is not always observed at the front (Hales et al., 2009) and seasonal mean climatologies of the cross-shelf distribution of chlorophyll do not appear to have significant frontal enhancement (Zhang et al., 2013). These studies highlight an important gap in our understanding of the Northeast U.S. Shelf ecosystem. Why is chlorophyll enhancement at the front absent from seasonal climatologies 
of the region when it has been observed in a number of studies at smaller time scales?

One potential explanation for this discrepancy is that frontal upwelling supports high productivity, but strong zooplankton grazing prevents the accumulation of phytoplankton biomass, thus eliminating the chlorophyll enhancement at seasonal time scales (Zhang et al., 2013). Under this scenario, biomass is transferred up the food chain to higher trophic levels and supports high secondary production rather than an increased standing stock of phytoplankton biomass. Even in the absence of a chlorophyll signal marking increased productivity, however, there may be alternative biological signatures that indicate a high-throughput system with both increased primary productivity and increased grazing.

Simultaneous high nutrient supply and zooplankton switching permits the coexistence of multiple phytoplankton types and results in a more even distribution of phytoplankton biomass between size classes (Chapter 4). Here, I will apply the principles derived from analysis of the size-structured model to a real ecosystem, and use patterns in phytoplankton community size structure to gain insight into the bottom-up and top-down controls on the community. My previous results suggest that under conditions of high nutrient availability and strong grazer impact, such as have been proposed to exist at the shelfbreak front, phytoplankton communities should have a more even distribution of biomass between size classes compared to communities with similar total biomass but lower nutrient supply and grazing rates. Phytoplankton community size structure is interesting because it provides a proxy for diversity. Cell size is an important structuring variable in phytoplankton communities and provides valuable information about a phytoplankter's biogeochemical characteristics (Chisholm, 1992).

High phytoplankton size class evenness results through the synergistic effects of coincident high nutrient availability and high zooplankton grazing impact (Fig. 5-1). Higher nutrient availability allows larger phytoplankton cells to flourish, since these cells have a much larger nutrient requirement due to their increased size. However, in the absence of any zooplankton, larger phytoplankton types will still get out-competed by smaller phytoplankton types, which are typically stronger competitors for nutrients and capable of fast nutrient uptake rates that reduce the environmental nutrient concentration below the point where larger cells can survive (Section 3.2.2). When zooplankton grazing has a strong impact on the community, however, the grazing pressure can prevent the smaller phytoplankton types from growing unchecked, either through switching or through com- 
munity compositional changes that focus increased grazing effort on smaller phytoplankton. These cooperating factors create an environment in which larger phytoplankton types can coexist with smaller phytoplankton types, resulting in a more diverse community. We have hypothesized that this increased diversity may provide a biological signal of simultaneous high productivity and high grazing (Section 4.3).

Phytoplankton biomass should increase under nutrient replete conditions, provided that the impact of zooplankton grazing on the community remains low (Fig. 5-1). Whether or not phytoplankton biomass increases when the community is subjected to simultaneous high nutrient availability and strong zooplankton grazing is a little more complicated. In the size structured model in the previous chapter, phytoplankton biomass remained low as nutrient supply was increased under strong grazing conditions (Section 4.3). That is because the model represents the highly idealized scenario in which zooplankton grazing is perfectly coupled to phytoplankton productivity. In other words, zooplankton grazing increases instantaneously in response to any increase in phytoplankton productivity. Phytoplankton biomass never accumulates because there is always enough grazing pressure to immediately transfer new biomass up the food chain. In the real world, however, zooplankton are unlikely to be perfectly coupled to phytoplankton. They require time to increase their numbers in response to more abundant food. Additionally, there are trophic levels above zooplankton that prevent zooplankton from increasing indefinitely, thus putting a limit on how much grazing pressure they can exert. Because of this, it is reasonable to expect that we would observe phytoplankton communities with both increased biomass and increased size class evenness. This is an important difference between our modeled phytoplankton communities and those that exist in the real world.

In this study, we will describe spatial patterns in phytoplankton biomass and community size structure from samples of the surface community collected in the shelfbreak front region of the Northeast U.S. Shelf in the spring and summer. These patterns will provide insight into the bottomup and top-down controls on phytoplankton communities and how the balance between these factors vary in space and time. We will focus on three primary questions.

1. Is phytoplankton biomass locally enhanced at the front compared to water masses on either side?

2. Does phytoplankton community size structure vary between water masses and between sea- 


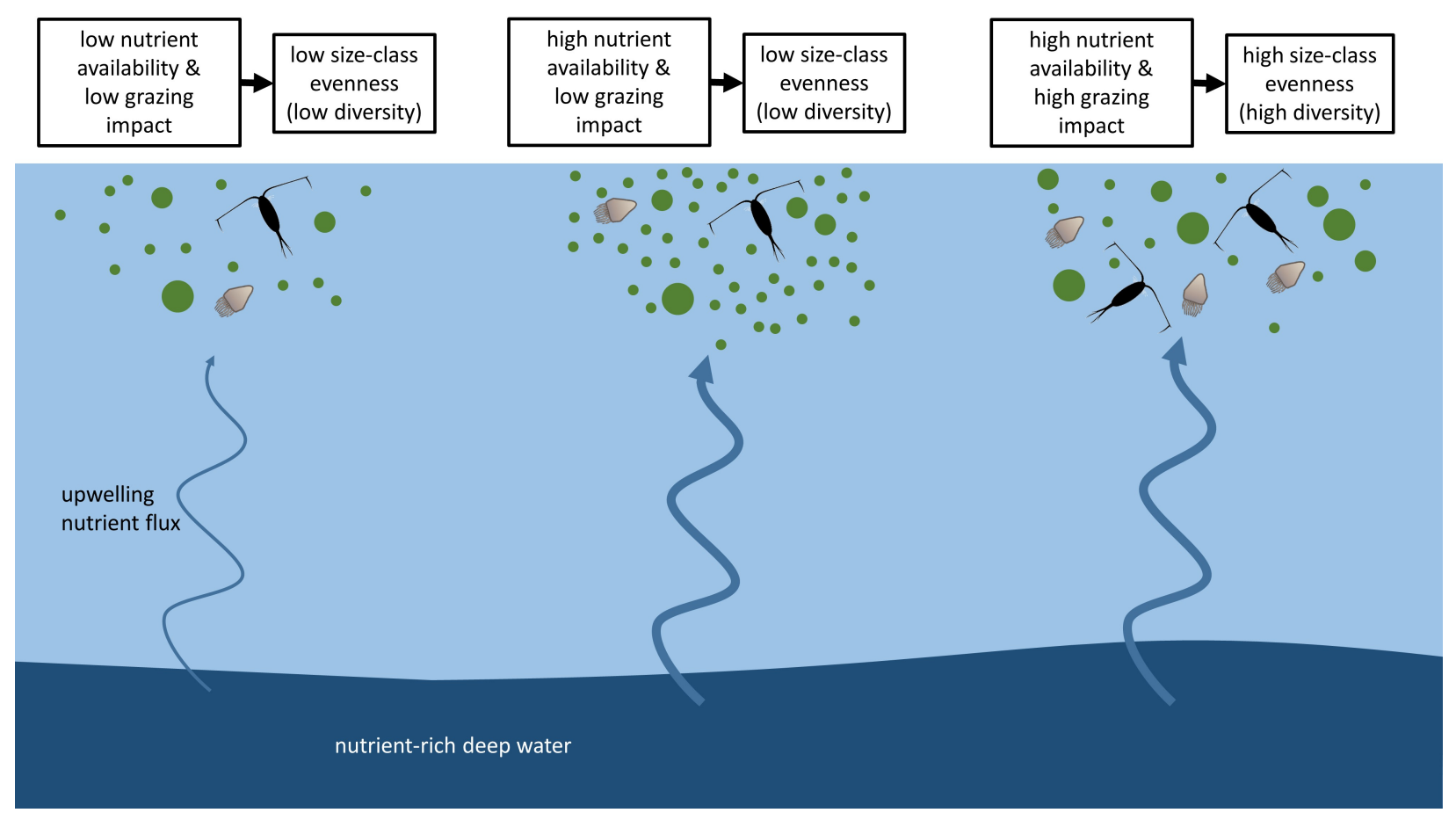

Figure 5-1: A conceptual model demonstrating how increased phytoplankton size class evenness may be generated under conditions of simultaneous high nutrient availability and high zooplankton grazing impact. 
sons?

3. Are the observed differences in size structure consistent with our hypothesis concerning increased size class evenness as a result of high nutrient availability and high grazing impact?

As part of a larger scientific effort, which includes a suite of process studies measuring primary productivity and zooplankton abundance and grazing rates, this study contributes to a growing understanding of the complex interplay between the physical and biological dynamics that play out in the Northeast U.S. Shelf ecosystem.

\subsection{Methods}

The Shelfbreak Productivity Interdisciplinary Research Operation at the Pioneer Array (SPIROPA) is a multi-institutional project with the aim of understanding the hydrodynamics and productivity of the shelfbreak front. Data collection for this project took place over three 14-day cruises to a sampling region approximately $100 \mathrm{~km}$ south of Martha's Vineyard, MA, in the vicinity of the Ocean Observing Initiative Pioneer Array (Fig. 5-2). The first cruise was conducted from April 16 to April 28, 2018, aboard the R/V Neil Armstrong (AR29). A second springtime cruise was conducted a year later from May 12 to May 24, 2019 aboard the R/V Ronald H. Brown (RB1904). The final cruise was conducted from July 5 to July 17, 2019 aboard the R/V Thomas G. Thompson (TN368). Our two springtime cruises (AR29 and RB1904) were scheduled to sample the ecosystem when ephemeral chlorophyll enhancement at the front was expected to be highest. Our summertime cruise (TN368) provided a contrast during the stratified summer months when enhancement in surface waters was expected to be reduced. Each cruise included multiple transects across the front, focusing on three locations - shelf water inshore of the front, at the front, and slope water offshore of the front. During these transects, an array of underway instruments continuously sampled the surface water over the course of the entire cruise.

\subsubsection{Underway instrument setup}

The underway setup included two types of cytometers used to obtain high-resolution measurements of phytoplankton community size structure. Pico- to nanoplankton were measured with an Attune 


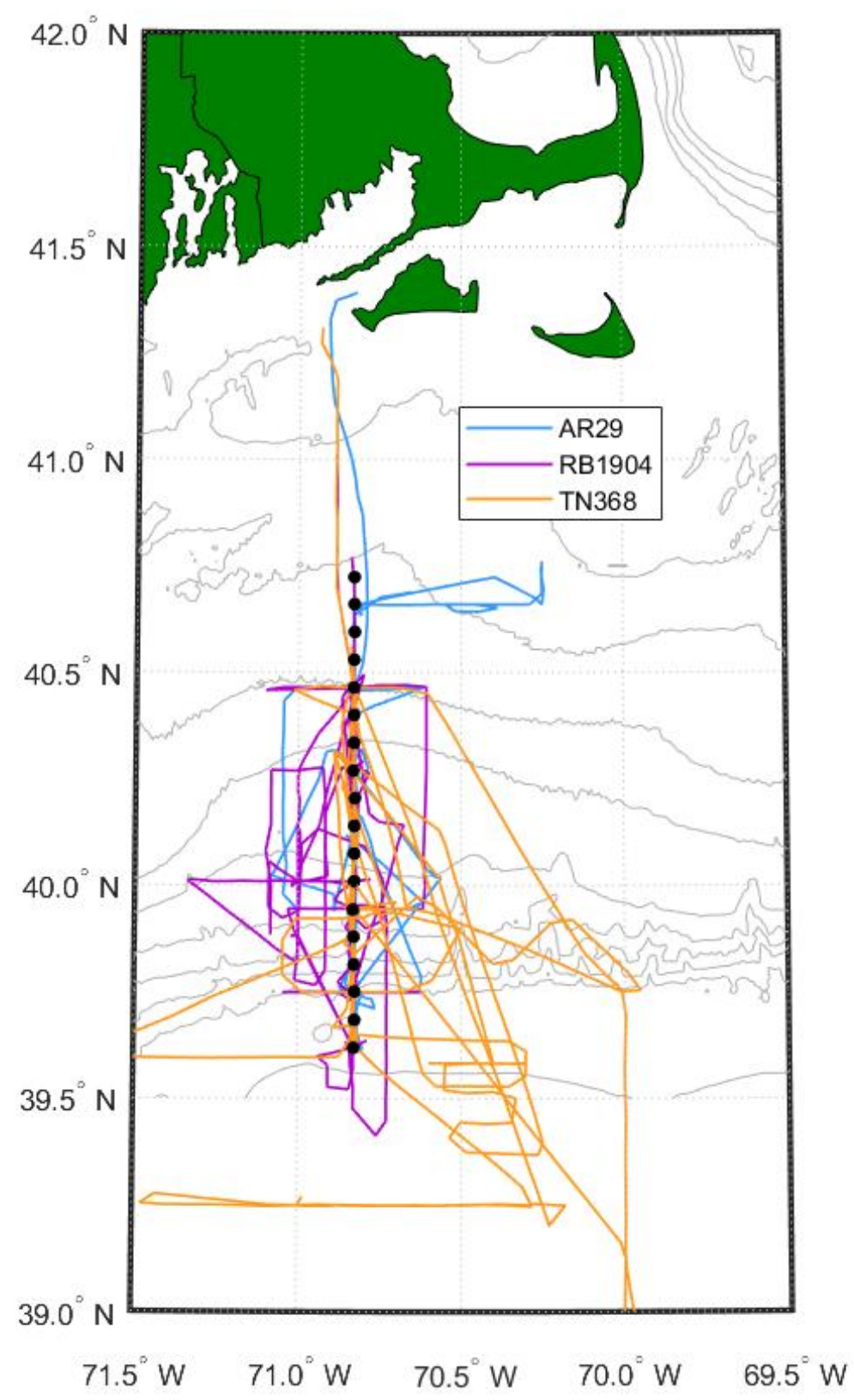

Figure 5-2: Location of the SPIROPA sampling transect (black) and the ship tracks for each of the three cruises. Bathymetry is shown in gray. 
NxT flow cytometer (Thermo Fisher Scientific) and nano- to microplankton were observed with an Imaging FlowCytobot (IFCB, McLane Research Laboratories). Two cytometers were necessary to characterize a wide size range of phytoplankton. The Attune has a dynamic range of approximately 0.5-15 $\mu \mathrm{m}$, and the IFCB has a range of approximately 7-150 $\mu \mathrm{m}$. We set up both instruments to automatically draw a sample from a continuous flow of seawater fed via diaphragm pump from the ship's sea chest. The Attune collected one 0.4-ml sample approximately every 2 minutes and the IFCB collected one 5-ml sample approximately every 26 minutes.

\subsubsection{Data calibration and collation}

For Attune observations, cell sizes were estimated from side angle light scattering. To minimize impacts of differences in instrument sensitivity and performance, side scattering observations were normalized to the mean side scattering signal of $1 \mu \mathrm{m}$ beads (Flow Check High Intensity Alignment Grade Particles, Polysciences) run periodically over the course of each cruise for RB1904 and TN368. On AR29, we used a different bead (Attune Performance Tracking Beads, Thermo Fisher Scientific) to normalize the data, and used a fixed ratio between the side scattering of these two bead types (determined post hoc) to convert into equivalent $1 \mu \mathrm{m}$ bead-normalized data. These normalized signals were then converted to cell volume based on a calibration curve generated from 12 phytoplankton cultures ranging in size from $1 \mu \mathrm{m}$ to $20 \mu \mathrm{m}$. Each culture was analyzed on the Attune and independently sized on a bead-calibrated Coulter Multisizer II (Beckman Coulter).

For IFCB observations, sizes of particles (individual cells, chains or colonies of cells) were estimated from images. Automated analysis following the scheme described by Sosik and Olson (2007), and updated in Sosik et al. (2020), was used to segment images into target and background pixels and compute geometric metrics. In particular, equivalent spherical diameter was computed from biovolume of imaged targets, as determined with the distance map algorithm of Moberg and Sosik (2012). For the spring 2018 cruise, the instruments were set to trigger based on the chlorophyll fluorescence signal of each particle, such that all measured particles had sufficiently high pigment concentration. However, for the two cruises in 2019, we triggered on both chlorophyll fluorescence and light scattering, which includes particles without chlorophyll. In the future, we will incorporate image analysis in order to exclude detrital particles (with or without chlorophyll).

Due to the different sampling frequencies between the two cytometers, all Attune samples within 
\pm 10 minutes of each IFCB sample were pooled and combined with the data from a single IFCB

file. Differences in sampling volume between the two instruments meant that the pooled Attune samples and the IFCB sample had approximately the same volume. A dynamic overlap region between the two instruments was determined by manually inspecting a subset of the data on each cruise and defining appropriate upper and lower limits for each instrument. A composite particle size distribution was constructed as follows. A fixed series of size bins was selected. For size bins smaller than the IFCB lower limit, the biovolume concentration within each size bin was determined by summing all the individual cell biovolume measurements within the size bin limits from the pooled Attune data and dividing by the total sampling volume of the pooled samples. For size bins larger than the upper limit of the Attune, the same procedure was conducted using only the IFCB data and the sampling volume of the IFCB. For those size bins within the overlap region, biovolume concentrations were determined by pooling measurements from both instruments and using the combined sampling volume of the pooled Attune samples and the IFCB sample. The total phytoplankton biovolume concentration in a collated sample was calculated by integrating over the composite particle size distribution. In the following analyses, biovolume concentration is used a proxy of phytoplankton biomass. I also used the particle size distributions to estimate the size diversity within a sample. Evenness (Eq. 4.5) and the Shannon Diversity Index (Eq. 4.6) were calculated using biovolume within each size bin.

\subsubsection{Categorical variables}

We defined a number of categorical variables to help structure the variance contained within the data set. The total biovolume integrated across the particle size distribution was divided into three size classes - picoplankton (0-2 $\mu \mathrm{m})$, nanoplankton $(2-20 \mu \mathrm{m})$, and microplankton $(20-150 \mu \mathrm{m})$. These size classes were selected to be consistent with previous definitions of plankton groups (Sieburth et al., 1978). Classically, the microplankton designation includes cells from 20-200 $\mu \mathrm{m}$. However, the IFCB has a functional upper detection limit of approximately $150 \mu \mathrm{m}$, and so we have chosen to truncate the microplankton size class. The salinity of each sample was used to define four different water masses across the shelfbreak. Salinity 32-34 was considered shelf water, 34-35 frontal water, 35-35.5 slope water, and greater than 35.5 warm-core ring (WCR) water. It is important to note that the high salinity water labeled as WCR can, in practice, come from a variety of Gulf Stream 
influenced sources. Finally, we defined four seasonal periods over the course of our three cruises (Table 5.1). The choice to consider the early and later portions of AR29 as two different seasonal periods was based on the presence of two distinct hydrographic regimes, described in more detail in the results section below.

\subsubsection{Statistical analyses}

To determine the statistical significance of differences in mean biovolume concentration between groups, we employed a one-factor analysis of variance (ANOVA) using a Bonferroni correction for post-hoc pairwise tests when the null hypothesis was rejected. Testing for differences in composition between samples, where the composition of a sample is defined as the three-element vector containing the fraction of total biovolume within each size class, was slightly more complex, given the additional constraint that a sample's composition vector must have unit sum. We developed a non-parametric randomization test to estimate the statistical significance of differences in composition between groups of samples, the details of which are described below.

Let $C_{j k}$ be the observed vector $k\left(k=1,2, \ldots, n_{j}\right)$ in region $j(j=1,2, \ldots, J)$ consisting of all the cell volume measurements in a given sample. Let $\mu_{j}$ be the three-element vector describing the mean composition (biovolume fraction of each size class) in region $j$. We tested the null hypothesis $H_{0}: \mu_{j}=\mu, j=1,2, \ldots, J$ of a common mean composition against the alternative hypothesis $H_{1}: \mu_{j} \neq \mu_{k}$ for at least one pair $j, k$. Let

$$
\hat{\mu}=\sum_{j=1}^{J} \sum_{k=1}^{n_{j}} C_{j, k} / \sum_{j=1}^{J} n_{j}
$$

be the estimate of the common mean composition under $H_{0}$ and

$$
\hat{\mu}_{j}=\sum_{k=1}^{n_{j}} C_{j, k} / n_{j}
$$

be the estimate of the mean composition for region $j$ under $H_{1}$. The goodness of fit under $H_{0}$ is measured by

$$
D_{0}=\sum_{j=1}^{J} \sum_{k=1}^{n_{j}}\left(C_{j, k}-\hat{\mu}\right)^{\prime}\left(C_{j, k}-\hat{\mu}\right)
$$




\begin{tabular}{||c|c|c||}
\hline Seasonal Period & Abbreviation & Dates \\
\hline Early Spring 2018 & Early SPR18 & April 16-21, 2018 \\
Late Spring 2018 & Late SPR18 & April 22-28, 2018 \\
Spring 2019 & SPR19 & May 12-24, 2019 \\
Summer 2019 & SUM19 & July 5-17, 2019 \\
\hline
\end{tabular}

Table 5.1: Date ranges and abbreviations for the four seasonal periods over the SPIROPA project. 
and the goodness of fit under $H_{1}$ is measured by

$$
D_{1}=\sum_{j=1}^{J} \sum_{k=1}^{n_{j}}\left(C_{j, k}-\hat{\mu}_{j}\right)^{\prime}\left(C_{j, k}-\hat{\mu}_{j}\right)
$$

Then the test statistic for evaluating $H_{1}$ against $H_{0}$ is $T=D_{0}-D_{1}$ with larger values of $T$ favoring $H_{1}$. The significance of the observed value of $T$ is evaluated via a randomization procedure in which the observations are pooled and randomly partitioned into $J$ groups of sizes $n_{1}, n_{2}, \ldots, n_{j}$. Estimates of $\hat{\mu}$ and $\hat{\mu}_{1}, \hat{\mu}_{2}, \ldots, \hat{\mu}_{j}$ are calculated from the randomized data, as well as the corresponding values of $D_{0}, D_{1}$, and $T$. This process was repeated a large number $(10,000)$ of times to generate a distribution of the test statistic, $T$. The significance of the observed value of $T$ is approximated as the proportion of values of $T$ larger than the observed value. In both our traditional ANOVA analyses and the randomization tests, the null hypothesis was evaluated at significance level $\alpha=0.05$.

\subsection{Results}

The variability in phytoplankton community size structure (Fig. 5-3) and total phytoplankton biovolume (Fig. 5-4 - 5-5) was high both within a given cruise and between cruises. We observed much higher phytoplankton biovolume concentrations on RB1904 than on either AR29 or TN368 (Fig. 54). This was also true for all three phytoplankton size classes individually. Over all cruises, most of the total biovolume was in the nanoplankton and microplankton size classes, with picoplankton having lower biovolume concentration compared to the larger size classes.

\subsubsection{Hydrographic context}

The shelfbreak front is a highly dynamic region with frequent influence from Gulf Stream rings. Identifying the hydrographic context over the course of each cruise is an important piece of understanding the observed patterns in phytoplankton biovolume and size structure. Advanced Very High-Resolution Radiometer (AVHRR) satellite imagery provides a picture of the sea surface temperature during our seasonal periods and revealed that the hydrodynamic structure of the front region varied significantly between cruises (Fig. 5-6). We identified two distinct regimes during our 


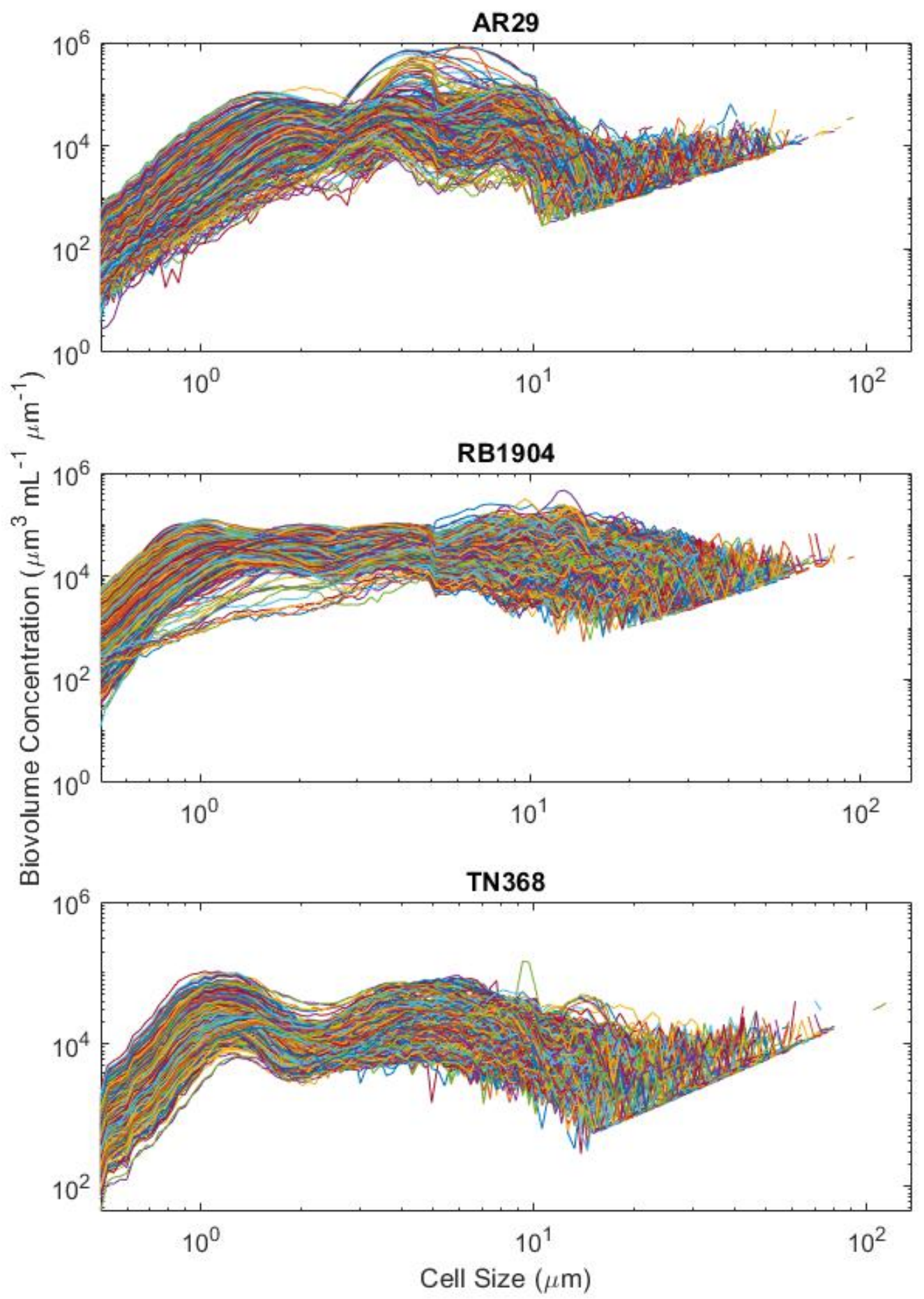

Figure 5-3: Composite particle size distributions for each of the three cruises. 

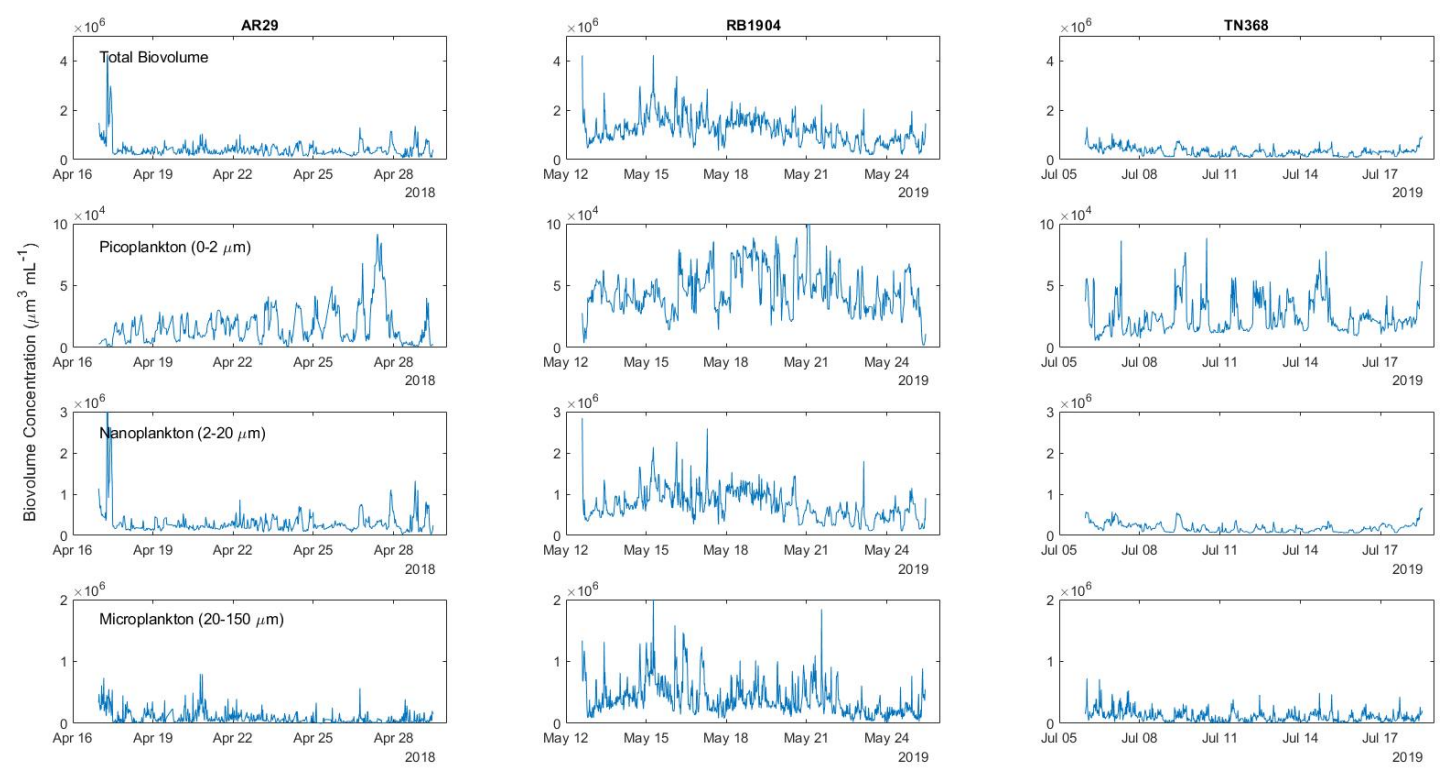

Figure 5-4: Time series for each cruise showing the total biovolume concentration and biovolume in each size class. Data are plotted on the same y-axis for each cruise. 

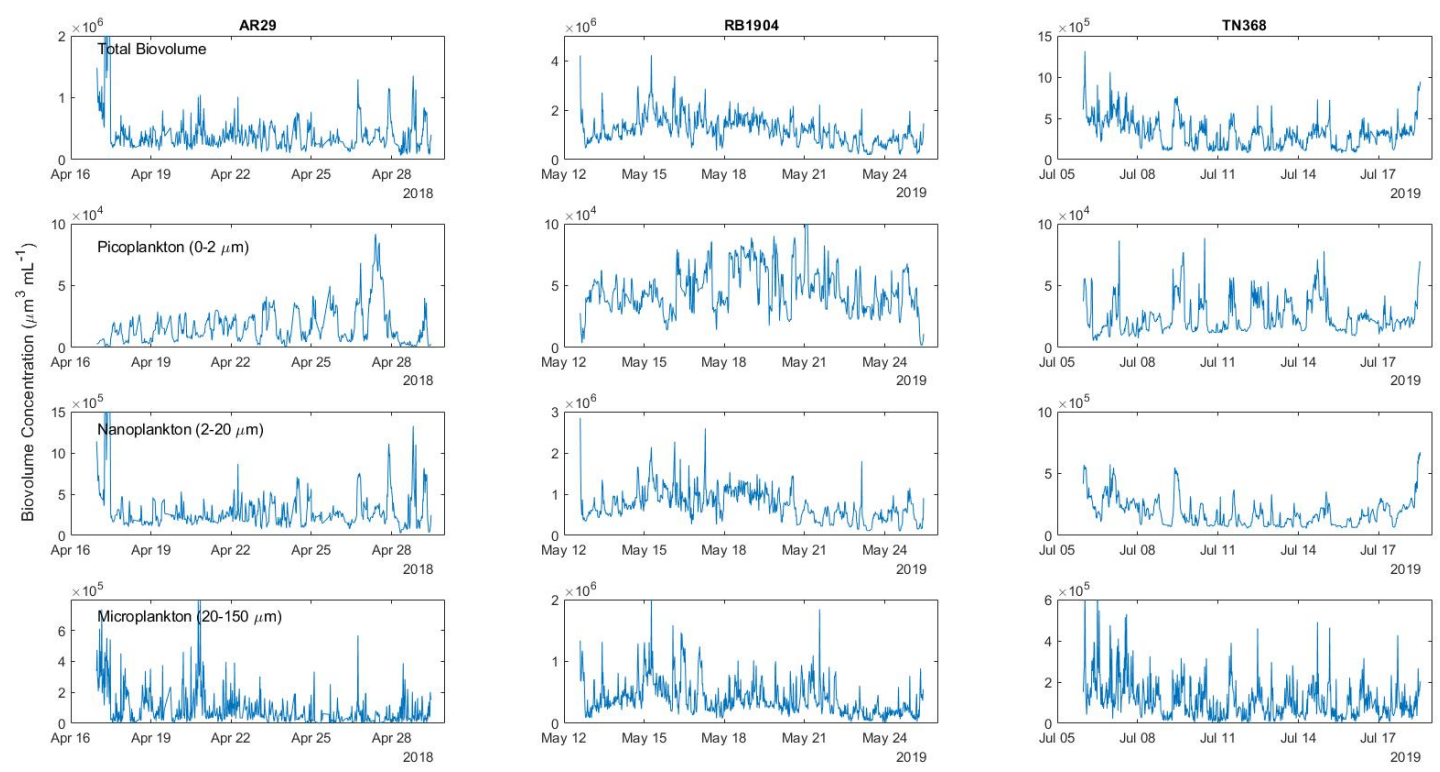

Figure 5-5: Time series for each cruise showing the total biovolume concentration and biovolume in each size class. Data are plotted using different y-axes for each cruise to better visualize variability within the time series. 
first spring cruise (SPR18). During the early portion of SPR18, the shelfbreak front was expressed as a density gradient between shelf water and slope water (Fig. 5-6a). However, later in the cruise, a WCR streamer entered the study area, during which time the slope water was subducted such that the front became a gradient between shelf water and WCR water (Fig. 5-6b). The presence of two distinct hydrographic regimes led us to decide to treat the cruise period before April 22, 2018 as a separate data set than the period after that date. A second unique feature of SPR18 that bears mentioning was the presence of a very large Phaeocystis bloom that was advected into the northern part of our study region. This bloom was characterized by abnormally high chlorophyll concentrations and was taxonomically identified using IFCB images. However, satellite imagery revealed that the center of this bloom was Georges Bank and the waters south of Nantucket, with a filament extending far enough south to cross our transect. We chose to exclude these samples from our analysis. During our second spring cruise (SPR19), a WCR was present in the southeast of our study area and propagated northwest over the course of the cruise. Interaction between this WCR and the shelfbreak front was accompanied by the formation of a shelfbreak front meander (Fig. 5-6c). Finally, our summer cruise (SUM19) had a WCR offshore of the front that drew a filament of cold and fresh shelf water offshore of the front to the east of our transect (Fig. 5-6d). This feature propagated eastward over our transect over the course of the cruise.

\subsubsection{Patterns in total phytoplankton biovolume}

During early SPR18, both front water and slope water had significantly higher biovolume concentration compared to shelf water, with slope water also significantly enhanced relative to front water (Fig. 5-7). This created a cross-shelf gradient of increasing phytoplankton biovolume offshore. WCR water, however, did not have increased biovolume concentration during this period. During late SPR18, we observed increased biovolume concentration at the front with no significant differences in biovolume concentration between any of the other water masses. SPR19 provided a second observation of the springtime period one year later. Similar to late SPR18, we observed significantly higher biovolume concentration at the front compared to shelf and slope water. However, unlike any time during SPR18, WCR water had the highest overall biovolume concentrations. We also

observed very large subsurface diatom blooms in the WCR water during this time period, likely produced by the upwelling of nutrient-rich deep Gulf Stream water Oliver et al., 2020. In contrast 

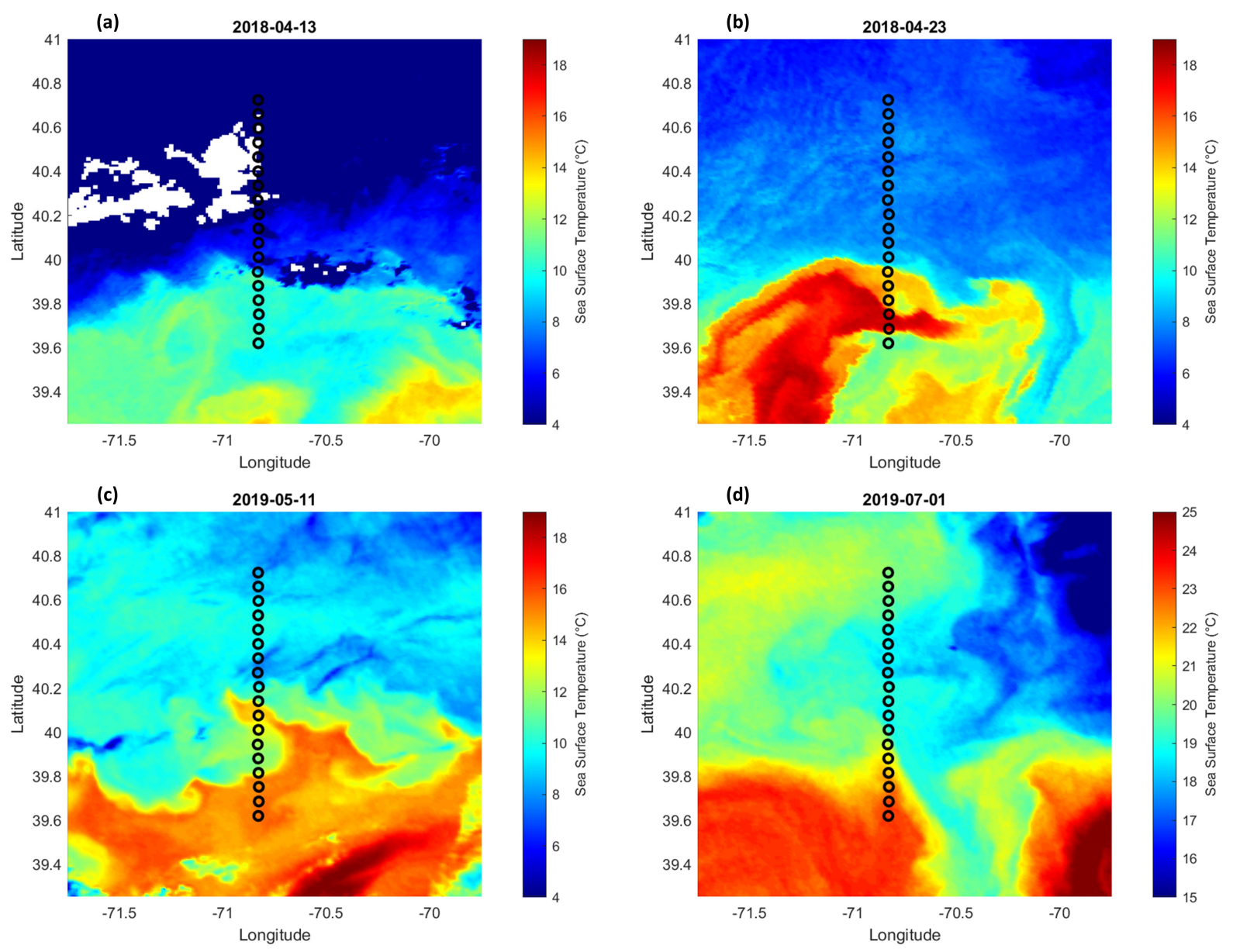

Figure 5-6: AVHRR satellite imagery showing sea surface temperature over the study area during (a) early SPR18, (b) late SPR18, (c) SPR19, and (d) SUM19. Transect station locations are indicated with black circles. 
to the spring cruises, SUM19 did not exhibit any local enhancement of phytoplankton biovolume at the front. Instead, we observed a cross-shelf gradient with significantly higher biovolume in the shelf water and lower biovolume offshore.

\subsubsection{Patterns in size structure}

We observed high variability in the biovolume fractions of pico, nano, and microplankton between water masses during all four seasonal periods (Fig. 5-8). The picoplankton biovolume fraction was consistently low, with differences between samples primarily driven by changes in the nanoplankton and microplankton biovolume fractions. SUM19 did show somewhat higher proportions of picoplankton compared to the spring cruises, however (Fig. 5-9). Early SPR18 was characterized by higher microplankton biovolume fractions in the slope water and high variability in the size structure of the front water (Fig. 5-11). Late SPR18 showed reduced fractions of microplankton in front water (Fig. 5-11), as well as WCR water with higher fractions of picoplankton (Fig. 5-9). SPR19 was more similar to early SRP18 than late SPR18, with the primary difference being consistently high fractions of microplankton in WCR water (Fig. 5-8).

The mean microplankton biovolume fraction was higher in front and slope water compared to shelf water during early SPR18 and SPR19 (Fig. 5-12). However, during late SPR18, we observed no increase in the microplankton fraction in the front and slope water. The opposite pattern was present during SUM19, with lower mean microplankton fractions offshore. WCR water showed higher mean microplankton fractions compared to other water masses only during SPR19.

Evenness and Shannon Diversity showed similar patterns (Fig. 5-13). Both were significantly higher at the front during early SPR18, but not during late SPR18 or SPR19. There were no significant differences between diversity metrics in any of the water masses during late SPR18. In SPR19, evenness and diversity was higher in shelf water. Evenness and diversity was also higher in shelf water during SUM19.

The relationship between total biovolume concentration and size structure diversity was not consistent (Table 5.2). We observed some cases (e.g. SPR19) in which the front had higher biovolume concentration, accompanied by significant size structure differences, compared to water masses with lower biovolume. We observed other cases (e.g. Late SPR18) in which water masses with higher biovolume concentration showed no difference in size structure compared to water masses 


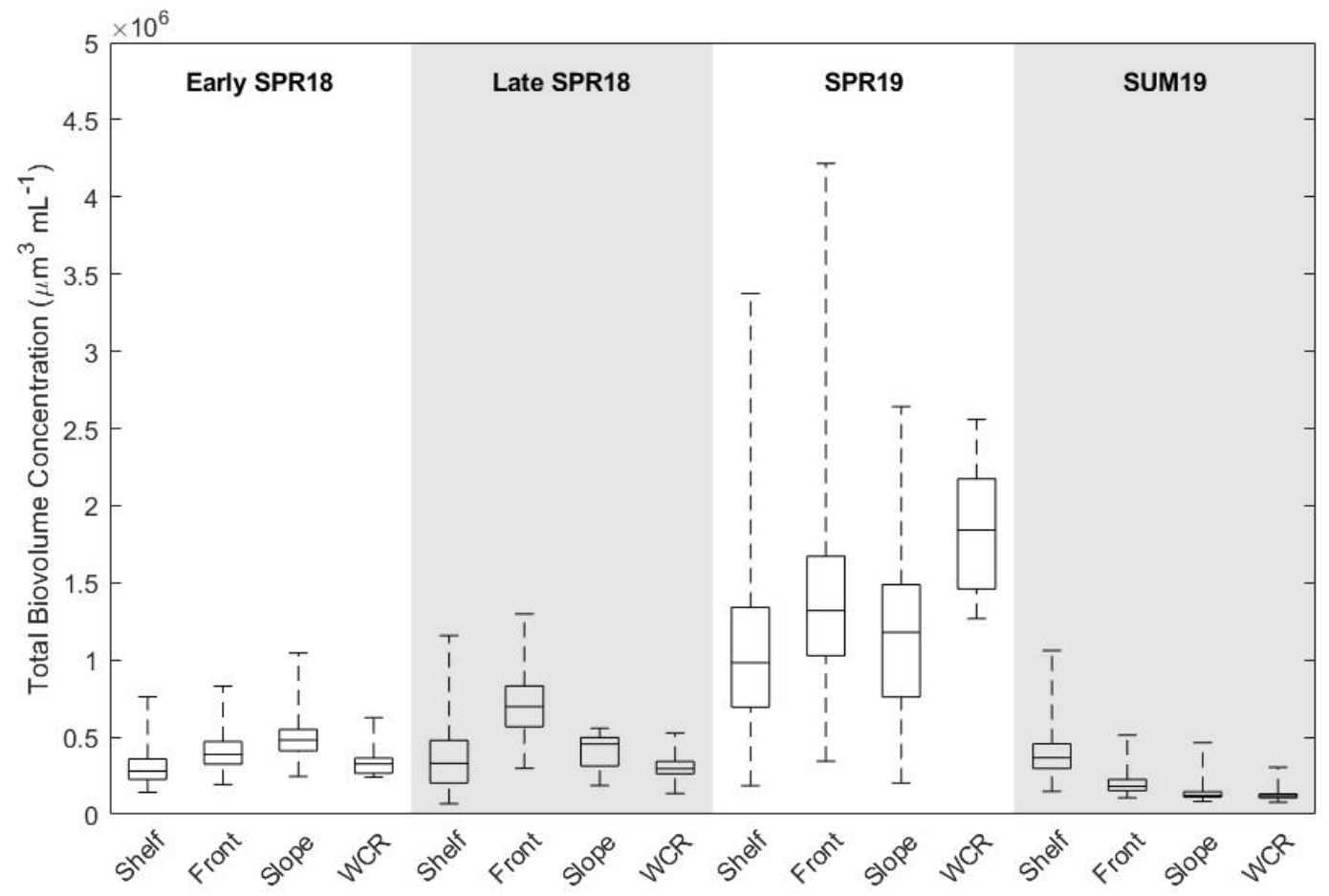

Figure 5-7: Distribution of total biovolume concentration in each water mass for all seasonal periods. 

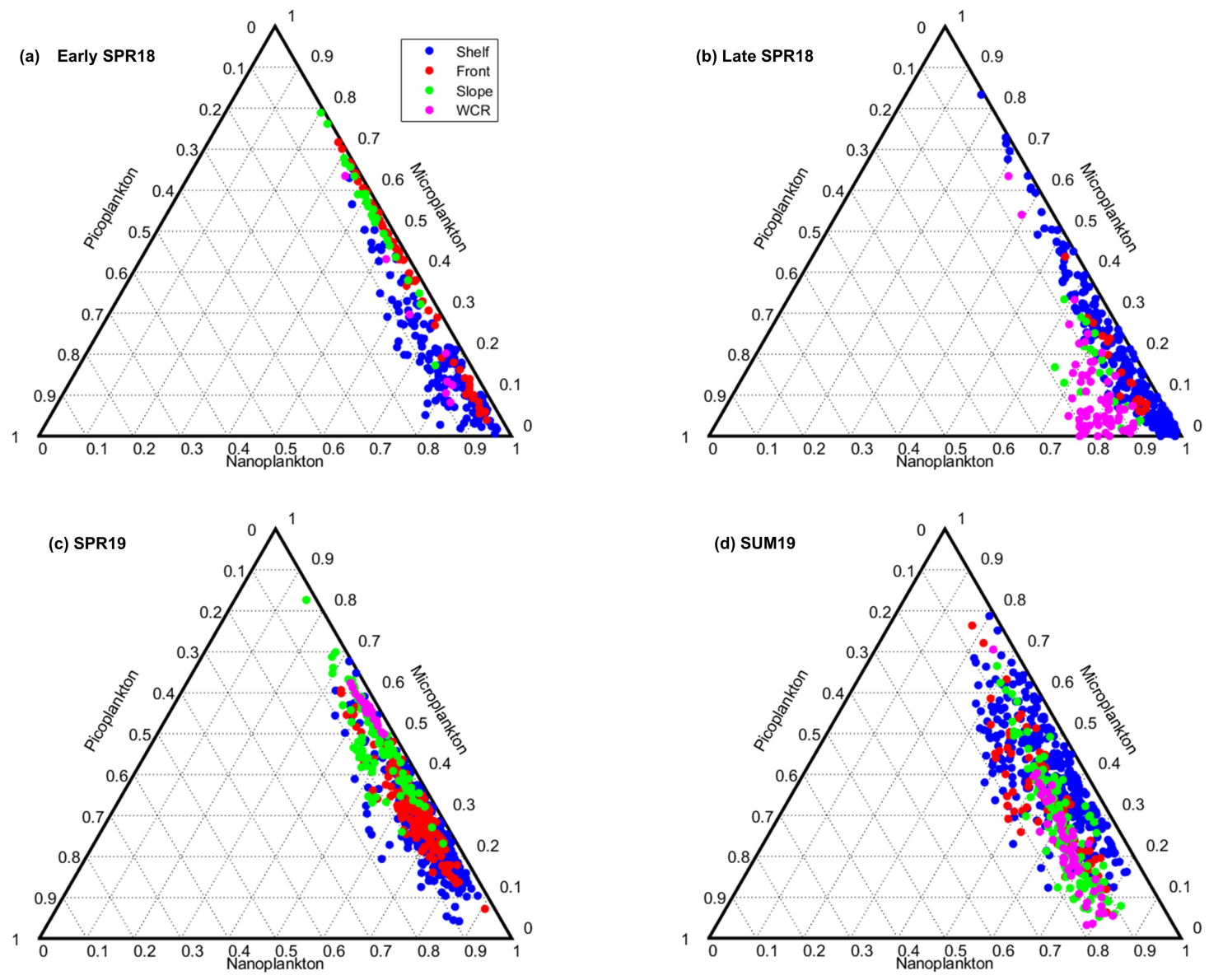

Figure 5-8: Simplex plots showing biovolume fraction of each size class. Samples are color coded by water mass. 
(a) Early SPR18

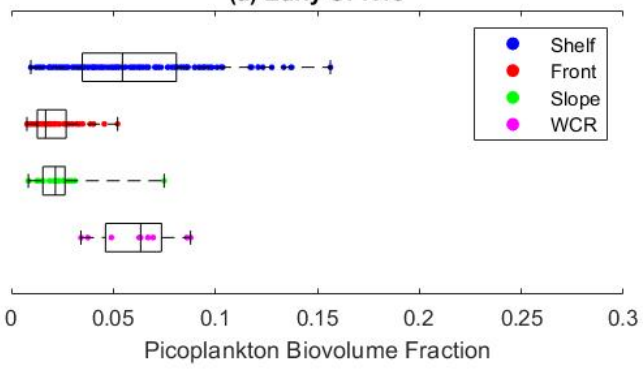

(c) SPR19

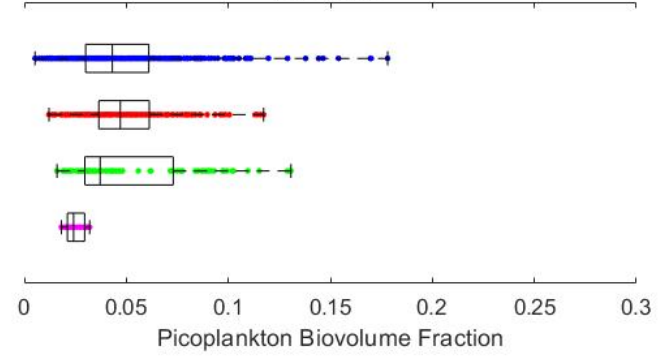

(b) Late SPR18

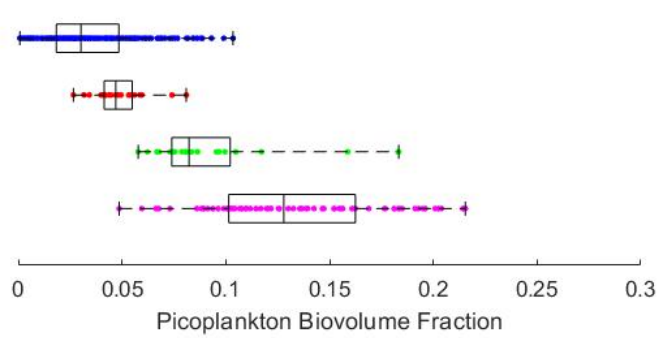

(d) SUM19

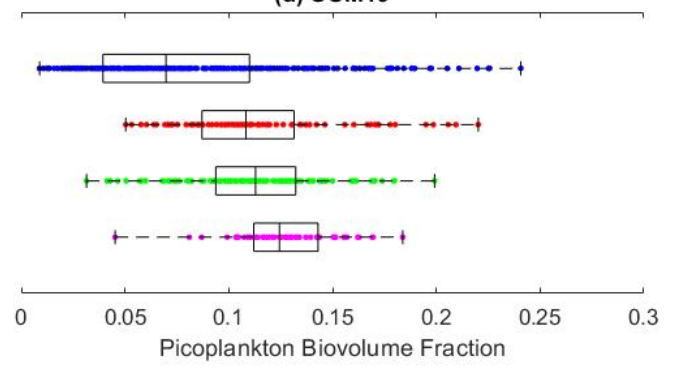

Figure 5-9: Distribution of picoplankton biovolume fraction for each water mass for all seasonal periods. Note that range of the $\mathrm{x}$-axis is limited to $(0,0.3)$. 

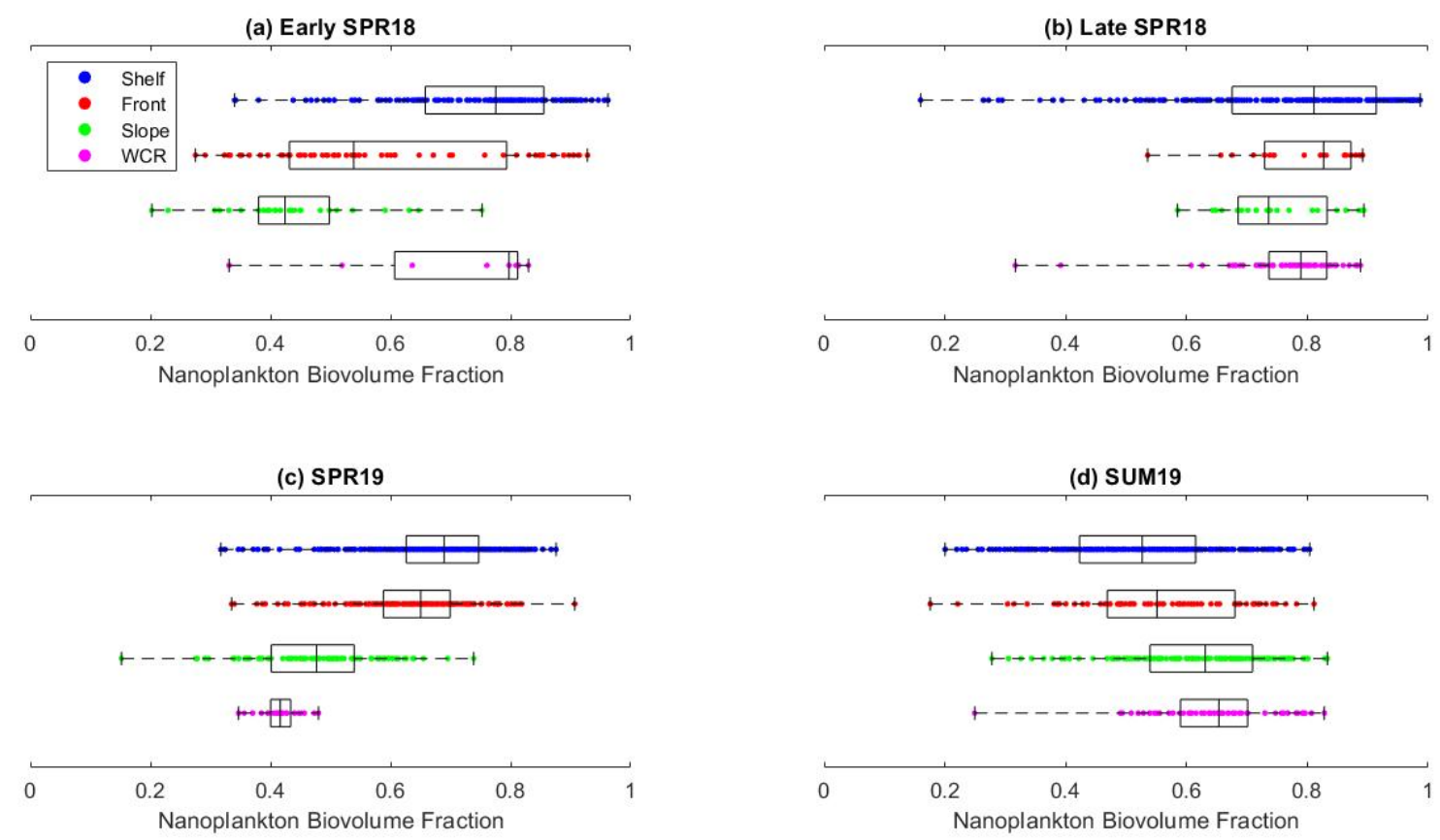

Figure 5-10: Distribution of nanoplankton biovolume fraction for each water mass for all seasonal periods. 


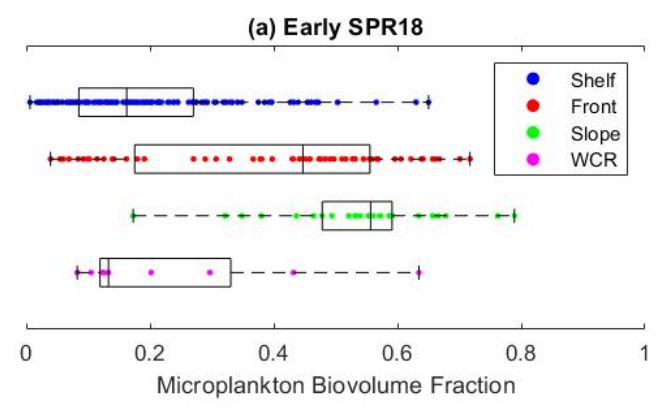

(c) SPR19

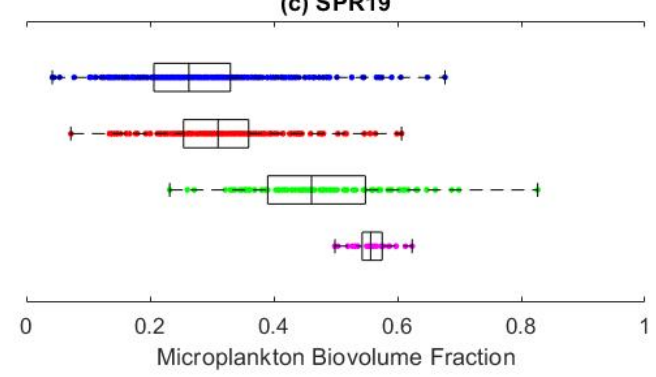

(b) Late SPR18

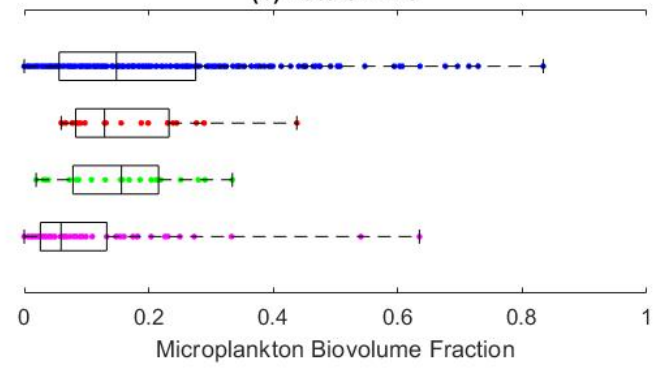

(d) SUM19

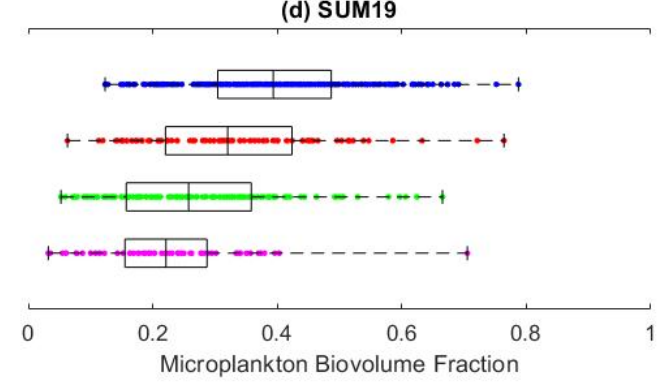

Figure 5-11: Distribution of microplankton biovolume fraction for each water mass for all seasonal periods. 

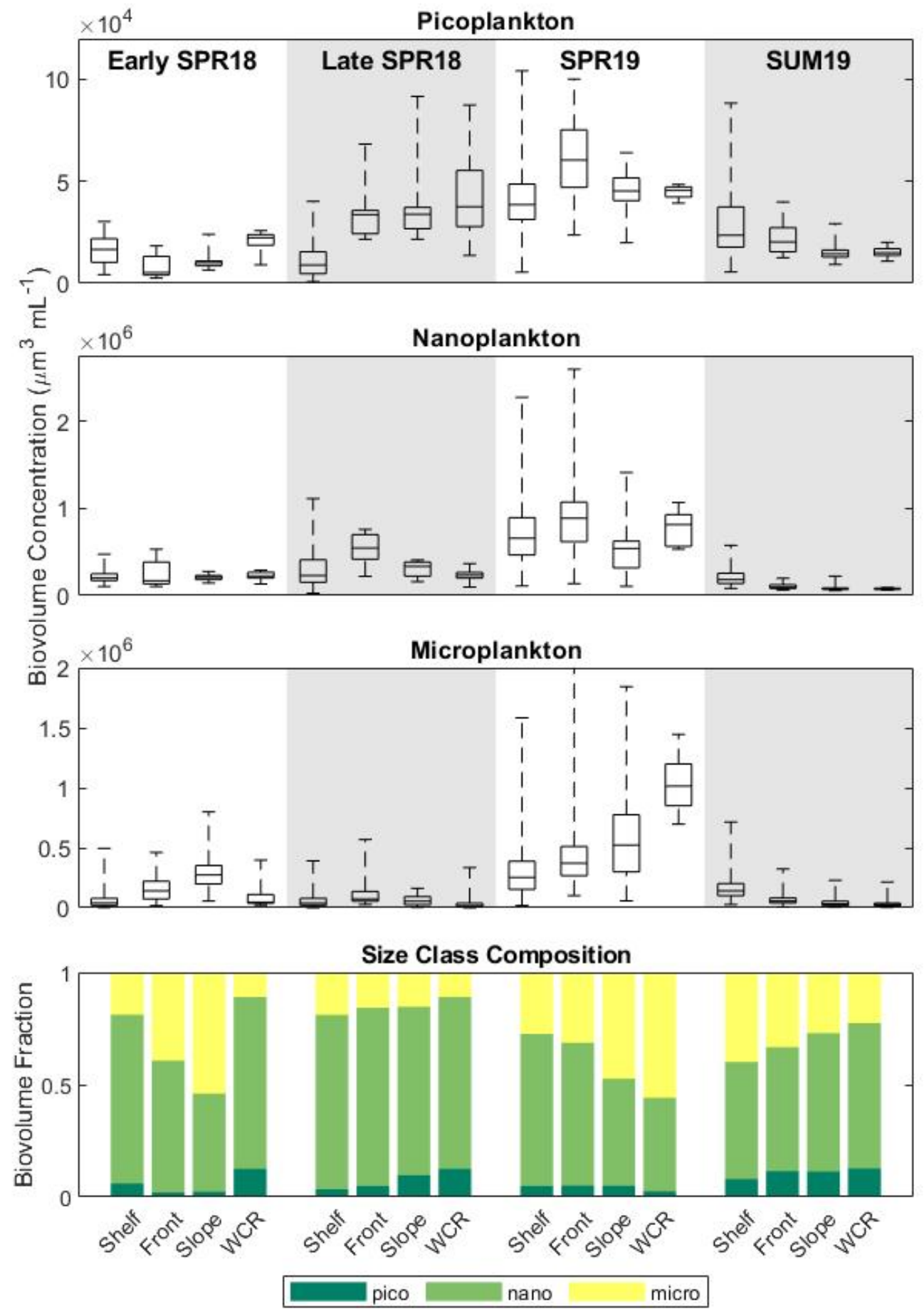

Figure 5-12: Distribution of biovolume concentrations in each size class, and the resulting mean size class composition, in each water mass. 

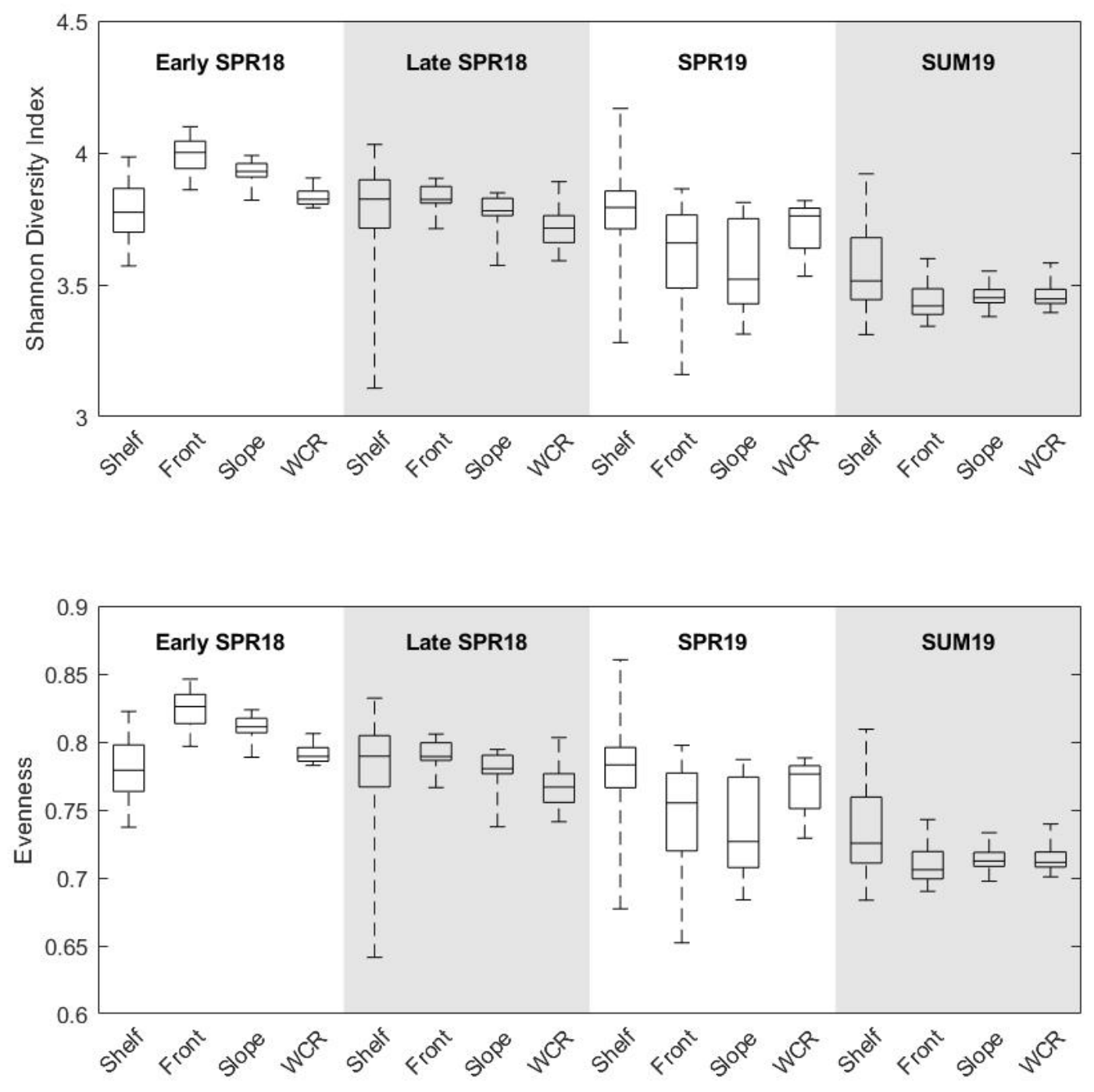

Figure 5-13: Distribution of Shannon Diversity Index and Evenness calculated from total biovolume in each size class. 
with lower biovolume. Similarly, higher biovolume concentration was sometimes associated with higher size class evenness and diversity (early SPR18) and sometimes not (late SPR18 and SPR19). During SUM19, the shelf waters had significantly higher biovolume concentration accompanied by significant differences in size structure and higher evenness and diversity.

\subsection{Discussion}

Local enhancement of phytoplankton biovolume concentration was observed near the shelfbreak front during both SPR18 and SPR19. During SPR18, we observed this feature evolving during the interval between late April and early May. This is consistent with previous observations of springtime frontal enhancement of chlorophyll concentration (Marra et al., 1982; O'Reilly and Busch, 1984; Ryan et al., 1999). Zhang et al. (2013), however, found no mean enhancement of chlorophyll at the front in seasonal climatologies. The authors of that study hypothesized that zooplankton grazing prevents the accumulation of phytoplankton biomass in the mean state. Our data suggest that phytoplankton enhancement is at least temporarily present at the shelfbreak front as a highly ephemeral feature. However, the enhancement may be intermittent enough (spatially or temporally) and small enough in amplitude that the small-scale enhancements we observed get averaged out in the mean state.

We observed no increased phytoplankton biovolume concentration near the front during SUM19. It should be noted, however, that this analysis was limited to phytoplankton communities at the surface that were sampled by our underway instrument array. During SUM19, we observed strong thermal stratification of the water column and subsurface maxima in phytoplankton chlorophyll during Conductivity, Temperature and Depth (CTD) and Video Plankton Recorder (VPR) casts. We sampled the subsurface phytoplankton communities from water collected during the CTD casts. Furthermore, Oliver et al. (2020) have described in detail the occurrence of subsurface diatom blooms observed in the WCR water during SPR19. Similar high-biomass phytoplankton commu-

nities may be associated with the high chlorophyll subsurface signal observed in SUM19. Future analysis of these preserved samples will reveal if increased phytoplankton biovolume concentration exists at depth and whether these communities show similar size structure differences as those observed in surface waters during the spring cruises. 


\begin{tabular}{||c|c|c|c|c||}
\hline $\begin{array}{c}\text { Seasonal } \\
\text { Period }\end{array}$ & $\begin{array}{c}\text { Enhanced Biovolume } \\
\text { Concentration }\end{array}$ & $\begin{array}{c}\text { Size Class } \\
\text { Composition Differences }\end{array}$ & $\begin{array}{c}\text { Higher } \\
\text { Diversity }\end{array}$ & $\begin{array}{c}\text { Higher } \\
\text { Evenness }\end{array}$ \\
\hline Early SPR18 & Front, Slope & Yes & Yes & Yes \\
Late SPR18 & Front & No & No & No \\
SPR19 & Front, WCR & Yes & No & No \\
SUM19 & Shelf & Yes & Yes & Yes \\
\hline
\end{tabular}

Table 5.2: Summary of statistically significant differences in water mass characteristics for each seasonal period. The second column lists which water masses had significantly higher biovolume concentrations compared to all other water masses for that period. Columns three through five indicate whether those water masses with higher biovolume concentration also had significantly different size class composition, Shannon Diversity Index, and Evenness. 
Interestingly, while increased biovolume concentration at the front was sometimes observed during the spring cruises, higher phytoplankton biovolume was not associated with consistent size structure changes. Over the three cruise periods in which we observed higher biovolume concentration at the front, we encountered cases when water masses with higher biovolume concentrations had significantly different size structure from surrounding water and cases when there were no size structure differences. We also observed water masses with similar biovolume concentrations that had significantly different size structure. When water masses with higher biovolume concentration did differ in size structure, we typically observed increased concentrations of nano and picoplankton and greater size class evenness offshore in the front and slope waters, compared to shelf water.

The observed patterns are partially consistent with our model-based predictions. In our simple conceptual model, high nutrient availability and strong grazing impact at the front results in increased evenness in front water compared to the shelf and slope waters. In fact, we observed increased evenness at the front only during early SPR18. During late SPR18, we observed no increase in evenness at the front despite seeing increased phytoplankton biovolume concentration. And during SPR19, there was increased biovolume concentration and higher microplankton fractions at the front, but no significant increase in evenness.

In the size-structured model described in Chapter 4, increased evenness was strictly related to higher relative proportions of larger phytoplankton size classes. This relationship arises from the fact that size classes are always added in order of increasing size as nutrient availability or grazing pressure is increased, and that the distribution of biomass is always monotonically decreasing from smallest to largest across the set of coexisting size classes. The model simulations suggest that increased microplankton biovolume fractions should be accompanied by greater evenness. However, the relationship between size class evenness and the relative fraction of the largest phytoplankton size classes in the Northeast U.S. Shelf ecosystem appears to be more complex. It is possible that under certain conditions, for example, microplankton may become the dominant size class in the phytoplankton community, a result which is impossible in the size-structured model. In that case, a high microplankton fraction would coincide with low size class evenness, as was observed at the front during SPR19.

There are a few key caveats that need to be addressed when discussing how the size-structured model can be applied to interpret the data presented in this chapter. First, the conceptual model 
(Fig. 5-1) that is here used to speculate about the relative nutrient availability and grazing impact on the phytoplankton community was derived based on model solutions at equilibrium. The time-scale required for the model to reach equilibrium was approximately one to several months. In contrast, on our SPIROPA cruises physical forcing was observed to vary on time scales of days to weeks. It is therefore likely that the empirical observations describe perturbations to the steady-state and not the stead-state itself. This is especially important to keep in mind for transient features such as the spring bloom, which evolve through space and time on time scales significantly shorter than the time scale of equilibrium observed in the model.

Additionally, it is important to consider that the size-structured model does not capture all the biological and physical variability that exists in the real world. One key difference is the potential for the empirical observations to be made during time periods when the phytoplankton community was light-limited rather than nutrient-limited. This was likely, for example, during early SPR18 when we observed nutrients at the surface to be in abundance. In that case, the conceptual model is less applicable since it excludes a key factor influencing phytoplankton community dynamics.

Another example is the simplified representation of zooplankton grazing in the model, which includes only one type of zooplankton. In the real world, grazer communities exist as many different zooplankton types with specific preferences and trophic relationships not just with phytoplankton, but with other grazers as well. Zooplankton grazing on other kinds of zooplankton creates the potential for trophic cascades, in which the grazing pressure on certain phytoplankton types is indirectly reduced when one zooplankton type consumes that phytoplankton type's specific grazer. These complex trophic relationships may lead to phytoplankton assemblages and size structures that cannot be produced in my simplified model. Moving forward, the development of more complex models for the Northeast U.S. Shelf ecosystem will be valuable for exploring the potential impact of the caveats discussed above.

Future analysis of the IFCB imagery will quantify the taxonomic diversity of the phytoplankton community in addition to the size diversity presented here. That data will give us additional insight and context for the patterns in size structure discussed in this chapter. Phytoplankton size is an extremely important component of functional diversity as it affects a number of key biogeochemical characteristics, including nutrient acquisition (Marañón et al., 2013) and light absorption (Finkel, 2001). There are other aspects of diversity, such as temperature dependence and elemental 
stoichiometry, which do not have simple allometric scaling laws (Tilman, 2001). Evaluating the taxonomic diversity of phytoplankton on the Northeast U.S. Shelf may help explain cases in which the size structure is surprising or unintuitive, considering our sized-based conceptual model.

Broadly speaking, the paradigm for pelagic phytoplankton communities is that some background concentration of picoplankton (sometimes referred to as a "lawn") exists at all times, and that phytoplankton biomass is increased by adding successively larger phytoplankton cells as nutrients are made more available (Chisholm, 1992). On the Northeast Shelf, however, location appears to play a significant role in modulating this paradigm. High microplankton biovolume was more likely to be found offshore in the slope and WCR waters, with lower concentrations on the shelf.

One possible explanation of these patterns is that the composition of the zooplankton grazer community across the shelf plays a significant role in determining phytoplankton community size structure. In Section 4.2, I modeled grazing as a constant top-down pressure that is distributed across different phytoplankton size classes based on their relative abundance. This is a very convenient simplification that allows us to parameterize the vast diversity of marine zooplankton into a single box represented in the model. However, in the real world, the taxonomic composition of the zooplankton community plays a significant role in determining phytoplankton community size structure, with different zooplankton assemblages producing shifts in phytoplankton community structure (Berquist et al., 1985). Historically, the copepod Calanus finmarchicus has been abundant on the U.S. Northeast Shelf, with lower concentrations offshore (Fleminger and Hulsemann, 1977). The presence of a large zooplankton grazer may partially explain why concentrations of microplankton were lower on the shelf. Determining the effect of zooplankton community composition on phytoplankton size structure is especially important considering recent modeling efforts that project significant declines in the abundance of $C$. finmarchicus on the Northeast U.S. Shelf as a result of warming sea surface temperatures (Grieve et al., 2017).

One of the advantages of collecting these data as part of the SPIROPA project (a large, interdisciplinary endeavour) is the availability of additional data sets we can leverage to provide the necessary context for our results. Other researchers participating in the SPRIOPA project measured zooplankton biomass and performed grazing incubations to estimate zooplankton grazing rates on each of our cruises. Measurements of the zooplankton grazing rate in different water masses will help us evaluate the hypothesis that increased grazing results in higher size class diversity. 
One drawback of this study is the limited number of replicate cruises, which included only one cruise that re-sampled the same season and then only for the spring. Additional data is needed to describe more years in both the spring and summer months to build an accurate picture of climatological mean states and interannual variability. The recently established Northeast U.S. Shelf Long-term Ecological Research (NES-LTER) Program will be greatly beneficial in this regard. The NES-LTER program samples the same transect used by the SPIROPA project four times over the seasonal cycle every year, and will continue to do so for the foreseeable future. These cruises include the same underway instrument setup described above and will produce many replicate data sets in the coming years. Long-term ecological sampling programs like this are uncommon, and their value in establishing climatological mean conditions and diagnosing ecological change over long term scales is immense.

The impact of climate change is of significant interest to scientists studying this region. Recently, an important regime shift was identified that has led to a significant increase in the number of warmcore rings impacting the shelf since about the year 2000 (Gangopadhyay et al., 2019). Our results suggest that interaction between the shelfbreak front and warm core rings is an important factor in determining phytoplankton productivity and community composition. An increase in the frequency of warm core rings may have had, and possibly continues to have, important consequence for the biological communities at the front and more broadly across the Northeast U.S. Shelf. Continued monitoring of frontal phytoplankton communities is important to identify and project future climaterelated changes to the ecosystem. 


\section{Chapter 6}

\section{Conclusions}

In this thesis, I have explored how specific zooplankton behaviors can have important impacts on the dynamics of marine planktonic ecosystems. First, I investigated the contribution of zooplankton DVM to the vertical flux of carbon as part of the biological pump. There has long been speculation about the significance of DVM to the biological pump, but quantitative estimates of its impact are rare. I estimated that DVM constitutes approximately $16 \%$ of the global carbon export flux associated with the biological pump and that the relative contribution of DVM is higher in subtropical latitudes. I also explored zooplankton switching, looking at how it behaves in nutrient-phytoplankton-zooplankton (NPZ) models with different levels of complexity. Zooplankton switching plays a significant role in promoting phytoplankton diversity because it allows competing phytoplankton types to coexist in situations that would otherwise lead to competitive exclusion. Stronger switching leads to phytoplankton communities with more coexisting size classes and a more even distribution of biomass between size classes. Along the way, I identified a particular characteristic of the Kill-the-Winner functional response, which I have termed synergistic grazing. Synergistic grazing occurs when the grazing rate on one phytoplankton type increases as the biomass of an alternative phytoplankton type increases. This characteristic can result in unintuitive model dynamics. Finally, I used the NPZ model results to develop a series of hypotheses concerning the relationship between nutrient supply and grazing and the biomass and diversity of phytoplankton communities. I compared these hypotheses against patterns of phytoplankton community size structure that we observed on the Northeast U.S. Shelf, finding that enhancement of phytoplankton biovolume at the shelfbreak front is common during the springtime, but these enhancement events 
are not associated with consistent changes in community size structure.

Much of the work in this thesis was motivated by the desire to quantify the effect of specific zooplankton behaviors in planktonic ecosystems. Both DVM and switching have been well documented by oceanographers for decades. Nevertheless, it is only recently that either one has started to make its way into biogeochemical models. This raises an interesting question: What obstacles exist that make it challenging to progress from a descriptive understanding of a biological phenomenon to a mathematical description that can be implemented in models on a global scale?

One reason that it may take time for additional biological detail to become mainstream in the modeling community is the natural progression in model complexity over time. Biological systems are messy things to try to model. They can be extremely complex and contain seemingly infinite levels of variability. It would be a mistake to immediately attempt to create a model that faithfully reproduces all this complexity in silico; the result would undoubtedly be a frightening mess of equations and parameters that is at least as inscrutable as the real world and far less informative to study. Instead, the approach of modelers is to abstract complex systems using a simple set of relationships that represent the fundamental drivers of the system. Importantly, we should not make models more complex simply for complexity's sake. A more complex model is only necessary if it becomes clear that a simple model is insufficient to answer a particular question. In this way, models used to study marine ecosystems progress from the very simple to the more complex in a very systematic manner as our mechanistic understanding of the system is developed and more complex questions are raised.

In some ways, the approach that I employed in developing the NPZ models in Chapters 3 and 4 of this thesis is a sort of microcosm for this progression of model complexity. I began with a simple diamond-shaped model and used it to study how zooplankton switching modulated the competitive relationship between just two phytoplankton types. However, it quickly became clear that the diamond-shaped food web model was insufficient for describing real world phytoplankton communities given how diverse those communities really are. The size-structured food web model, built using a few simple allometric scaling laws, was the next logical step in building up model complexity. This model allowed me to investigate how those competitive relationships between pairs of phytoplankton types played out in a more complete and representative community. In turn, the size-structured model elucidated the relationship between switching and phytoplankton 
diversity, providing important context for global biogeochemical models that describe how switching impacts diversity on a global scale.

Similarly, my work to quantify the carbon export flux associated with zooplankton DVM was very much an attempt to answer the question of how much biological detail is needed when modeling the biological pump. Is a simpler model (i.e., one that does not include DVM) sufficient? I found that when I simulated the model with DVM, it increased the modeled global carbon export flux by $14 \%$ relative to model simulations that did not include DVM. This provided strong evidence that including DVM in global models is a necessary endeavour or we risk significantly underestimating the carbon export flux of the biological pump. We compared our model to field measurements of both zooplankton biomass and DVM-mediated flux values to build a strong argument for the validity of our findings.

Which brings me to a second obstacle to incorporating specific zooplankton behaviors into models - sometimes behaviors that are well documented still lack data describing their magnitude and variability over large spatial or temporal scales. For example, the DVM model was highly sensitive to the parameter describing the fraction of the zooplankton community that participated in DVM. For our simulations, we used a value of $50 \%$, but a literature review revealed that that value can range from nearly $0 \%$ to almost $100 \%$ depending on the taxonomic composition of the zooplankton assemblage. Without good observations describing the variability of this parameter between regions and over the seasonal cycle, these kinds of models are useful for theoretical and diagnostic type studies, but cannot be used effectively to predict future outcomes.

My work on zooplankton switching also revealed that phytoplankton diversity in my models was highly sensitive to the parameter describing the strength of switching in the grazer community. This parameter is especially difficult to characterize because it describes an emergent behavior of a community and is not easily measurable in real ecosystems. This is exemplified by the discussion in Section 3.3 that highlights how switching can be viewed as either arising from behavioral changes in individual zooplankton or from compositional changes to the zooplankton assemblage. Indeed, at the time I wrote that chapter, I was unable to find a single attempt to quantify switching in a real ecosystem, despite many studies documenting that switching was an important process in promoting phytoplankton diversity.

These challenges underscore the importance of modelers and empiricists communicating clearly 
about the needs of their particular fields. As useful as models are for understanding the patterns we observe in the real world, field observations and experiments are equally critical for helping constrain and parameterize models. The work that I have done in this thesis provides evidence that zooplankton DVM and switching are important processes in driving planktonic ecosystem dynamics. However, there remains much work to do before these phenomena can be accurately incorporated into the kinds of global biogeochemical models that are used to simulate global nutrient cycling, phytoplankton biogeography, or climate processes. The first priority moving forward is to develop standardized methods for observing community level characteristics (e.g. strength of switching or the fraction of the zooplankton community participating in DVM) so that we can effectively measure the variability of these key model parameters in different ecosystems and biogeochemical regimes. More complex models that include zooplankton behaviors are highly sensitive to these parameters and without good information to help appropriately parameterize these models, their application will remain limited to theoretical questions.

I hope that the legacy of this work is to inspire others to continue to think critically about all the fascinating and intricate behaviors that have been observed in zooplankton communities. What are the potential impacts of those behaviors? How can we abstract those behaviors and study them in a model? What data is needed to constrain our understanding of the behavior and characterize its variability? The microscopic world of phytoplankton and zooplankton is rich in both diversity and complexity, with implications for the global biosphere. It is a daunting task to try to quantify just how the individual choices of uncountable numbers of tiny organisms can echo with global-scale impacts, but it is one that I have found endlessly fascinating and rewarding. I hope others will as well. 


\section{Appendix A}

\section{Proof of competitive exclusion of}

\section{phytoplankton $P_{2}$ under Cases 1 and 3 of the diamond-shaped food web model}

In this appendix we show that the second phytoplankton species, $P_{2}$, which is also the poorer competitor in the absence of zooplankton grazing, cannot invade the $N-P_{1}-Z$ system at equilibrium in Cases 1 and 3 of Table 3.1 in Section 3.2.2. In particular, we show that $r$, as given by Eq. 3.10, is negative in these cases.

By assumption, $\mu_{1}>\mu_{2}$ and $k_{1}<k_{2}$. As a result

$$
\frac{\mu_{2} N}{k_{2}+N}<\frac{\mu_{1} N}{k_{1}+N}
$$

for all $N>0$. It follows that

$$
\frac{\mu_{2} N^{*}}{k_{2}+N^{*}}-m_{p}-D<\frac{\mu_{1} N^{*}}{k_{1}+N^{*}}-m_{p}-D
$$

where $N^{*}$ is the equilibrium nutrient concentration of the $N-P_{1}-Z$ system given by Eqs. 3.7 and 3.8. The right side of inequality (A.2) is equivalent to $G_{1}\left(P_{1}^{*}, 0\right) Z^{*} / P_{1}^{*}$, and therefore always positive assuming positive values of $P_{1}^{*}$ and $Z^{*}$. 
Now, since $\rho_{2}>\rho_{1}>0$ in Cases 1 and 3 ,

$$
\frac{\mu_{2} N^{*}}{k_{2}+N^{*}}-m_{p}-D<\frac{\rho_{2}}{\rho_{1}}\left(\frac{\mu_{1} N^{*}}{k_{1}+N^{*}}-m_{p}-D\right)
$$

and

$$
\frac{\mu_{2} N^{*}}{k_{2}+N^{*}}-m_{p}-D-\frac{\rho_{2}}{\rho_{1}}\left(\frac{\mu_{1} N^{*}}{k_{1}+N^{*}}-m_{p}-D\right)<0,
$$

and

$$
\frac{\mu_{2} N^{*}}{k_{2}+N^{*}}-m_{p}-D-\frac{\rho_{2} g_{\max }\left[\frac{k_{s a t}+\rho_{1} P_{1}^{*}}{\rho_{1} g_{\max }}\left(\frac{\mu_{1} N^{*}}{k_{1}+N^{*}}-m_{p}-D\right)\right]}{k_{\text {sat }}+\rho_{1} P_{1}^{*}}<0 .
$$

The term in square brackets in inequality (A.5) is, by Eq. 3.9,

$$
\frac{k_{\text {sat }}+\rho_{1} P_{1}^{*}}{\rho_{1} g_{\max }}\left(\frac{\mu_{1} N^{*}}{k_{1}+N^{*}}-m_{p}-D\right)=Z^{*}
$$

Upon substitution back into inequality (A.5) we have

$$
\frac{\mu_{2} N^{*}}{k_{2}+N^{*}}-m_{p}-D-\frac{\rho_{2} g_{\max } Z^{*}}{k_{\text {sat }}+\rho_{1} P_{1}^{*}}<0
$$

Inequality (A.7), along with Eq 10, guarantees that $r<0$ for all positive parameter values in Cases 1 and 3 , as $\alpha=1$ for these cases. 


\section{Appendix B}

\section{References}

Adjou, M., Bendtsen, J., \& Richardson, K. (2012). Modeling the influence from ocean transport, mixing and grazing on phytoplankton diversity. Ecological Modelling, 225, 19-27.

Al-Mutairi, H., \& Landry, M. R. (2001). Active export of carbon and nitrogen at Station ALOHA by diel migrant zooplankton. Deep Sea Research Part II: Topical Studies in Oceanography, 48, 2083-2103.

Anderson, T. R., \& Gentleman, W. C. (2012). The legacy of Gordon Arthur Riley (1911-1985) and the development of mathematical models in biological oceanography. Journal of Marine Research, 70, 1-30.

Armstrong, R. A. (1979). Prey species replacement along a gradient of nutrient enrichment: A graphical approach. Ecology, 60, 76-84.

Armstrong, R. A. (1994). Grazing limitation and nutrient limitation in marine ecosystems: Steady state solutions of an ecosystem model with multiple food chains. Limnology and Oceanography, 39, 597-608.

Armstrong, R. A. (1999). Stable model structures for representing biogeochemical diversity and size spectra in plankton communities. Journal of Plankton Research, 21, 445-464.

Armstrong, R. A., Lee, C., Hedges, J. I., Honjo, S., \& Wakeham, S. G. (2002). A new, mechanistic model for organic carbon fluxes in the ocean based on the quantitative association of POC with ballast minerals. Deep Sea Research Part II: Topical Studies in Oceanography, 49, 219236.

Atkinson, A., Ward, P., \& Murphy, E. J. (1996). Diel periodicity of subantarctic copepods: Relationships between vertical migration, gut fullness and gut evacuation rate. Journal of Plankton Research, 18, 1387-1405.

Aumont, O., Ethé, C., Tagliabue, A., Bopp, L., \& Gehlen, M. (2015). PISCES-v2: an ocean biogeochemical model for carbon and ecosystem studies. Geoscientific Model Development Discussions, 8, 2465-2513.

Aumont, O., Maury, O., Lefort, S., \& Bopp, L. (2018). Evaluating the potential impacts of the diurnal vertical migration by marine organisms on marine biogeochemistry. Global Biogeochemical Cycles, 32, 1622-1643.

Baird, M. E., \& Suthers, I. M. (2007). A size-resolved pelagic ecosystem model. Ecological Modeling, 203. 
Baudrot, V., Perasso, A., Fritsch, C., Giraudoux, P., \& Raoul, F. (2016). The adaptation of generalist predators' diet in a multi-prey context: Insights from new functional responses. Ecology, 97, $1832-1841$.

Bautista, B., \& Harris, R. P. (1992). Copepod gut contents, ingestion rates and grazing impact on phytoplankton in relation to size structure of zooplankton and phytoplankton during a spring bloom. Marine Ecology Progress Series, 82, 41-50.

Berquist, A. M., Carpenter, S. R., \& Latino, J. C. (1985). Shifts in phytoplankton size structure and community composition during grazing by contrasting zooplankton assemblages. Limnology and Oceanography, 30, 1037-1045.

Bianchi, D., Galbraith, E. D., Carozza, D. A., Mislan, K. A. S., \& Stock, C. A. (2013a). Intensification of open-ocean oxygen depletion by vertically migrating animals. Nature Geoscience, $6,545-548$.

Bianchi, D., \& Mislan, K. A. S. (2016). Global patterns of diel vertical migration times and velocities from acoustic data. Limnology and Oceanography, 61, 353-364.

Bianchi, D., Stock, C., Galbraith, E. D., \& Sarmiento, J. L. (2013b). Diel vertical migration: Ecological controls and impacts on the biological pump in a one-dimensional ocean model. Global Biogeochemical Cycles, 27, 478-491.

Box, G. E. P. (1979). Robustness in statistics. Academic Press.

Boyd, P. W., \& Trull, T. W. (2007). Understanding the export of biogenic particles in oceanic waters: Is there consensus? Progress in Oceanography, 72, 276-312.

Buesseler, K. O., \& Boyd, P. W. (2009). Shedding light on processes that control particle export and flux attenuation in the twilight zone of the open ocean. Limnology and Oceanography, $54,1210-1232$.

Buitenhuis, E., Le Quére, C., Aumont, O., Beaugrand, G., Bunker, A., Hirst, A., Ikeda, T., O’Brien, T., Piontkovski, S., \& Straiel, D. (2006). Biogeochemical fluxes through mesozooplankton. Global Biogeochemical Cycles, 20, 1-18.

Butler, G. J., \& Wolkowicz, G. S. K. (1985). A mathematical model of the chemostat with a general class of functions describing nutrient uptake. SIAM Journal on Applied Mathematics, 45, $138-151$.

Butler, G. J., \& Wolkowicz, G. S. K. (1986). Predator-mediated competition in the chemostat. Journal of Mathematical Biology, 24, 167-191.

Cadier, M., Sourisseau, M., Gorgues, T., Edwards, C. A., \& Memery, L. (2017). Assessing spatial and temporal variability of phytoplankton communities' composition in the Iroise Sea ecosystem (Brittany, France): A 3D modeling approach: Part 2: Linking summer mesoscale distribution of phenotypic diversity to hydrodynamism. Journal of Marine Systems, 169, 111-126.

Chassot, E., Bonhommeau, S., Dulvy, N. K., Mélin, F., Watson, R., Gascuel, D., \& Le Pape, O. (2010). Global marine primary production constrains fisheries catches. Ecology Letters, 13, 495-505.

Chen, B., Smith, S. L., \& Wirtz, K. W. (2019). Effect of phytoplankton size diversity on primary productivity in the North Pacific: trait distributions under environmental variability. Ecology Letters, 22, 56-66.

Chisholm, S. W. (1992). Phytoplankton Size. In P. G. Falkowski, A. D. Woodhead, \& K. Vivirito (Eds.), Primary productivity and biogeochemical cycles in the sea (pp. 213-237). Springer.

Cock, M. J. W. (1978). The assessment of preference. Journal of Animal Ecology, 47, 805-816.

Cramer, N. F., \& May, R. M. (1972). Interspecific competition, predation and species diversity: a comment. Journal of Theoretical Biology, 34, 289-293.

Dam, H. G., \& Peterson, W. T. (1988). The effect of temperature on the gut clearance rate constant of planktonic copepods. Journal of Experimental Marine Biology and Ecology, 123, 1-14. 
Doney, S. C., Glover, D. M., \& Najjar, R. G. (1996). A new coupled, one-dimensional biologicalphysical model for the upper ocean: Applications to the JGOFS Bermuda Atlantic Timeseries Study (BATS) site. Deep Sea Research Part II: Topical Studies in Oceanography, 43, 591-624.

Dutkiewicz, S., Cermeno, P., Jahn, O., Follows, M. J., Hickman, A., Taniguchi, D., \& Ward, B. (2020). Dimensions of marine phytoplankton diversity. Biogeosciences, 17, 609-634.

Egilmez, H. I., \& Morozov, A. Y. (2016). Tri-trophic plankton models revised: Importance of space, food web structure and functional response parametrisation. Mathematical Modelling of Natural Phenomena, 11, 16-33.

Eppley, R. W., \& Peterson, B. J. (1979). Particulate organic matter flux and planktonic new production in the deep ocean. Nature, 282, 677-680.

Evans, G. T., \& Parslow, J. S. (1985). A model of annual plankton cycles. Biological Oceanography, 3, 327-347.

Falkowski, P. G., Barber, R. T., \& Smetacek, V. (1998). Biogeochemical controls and feedbacks on ocean primary production. Science, 281, 200-206.

Fasham, M. J. R., Ducklow, H. W., \& McKelvie, S. M. (1990). A nitrogen-based model of plankton dynamics in the oceanic mixed layer. Journal of Marine Research, 48, 591-639.

Field, C. B., Behrenfeld, M. J., Randerson, J. T., \& Falkowski, P. (1998). Primary production of the biosphere: integrating terrestrial and oceanic components. Science, 281, 237-240.

Finkel, Z. V. (2001). Light absorption and size scaling of light-limited metabolism in marine diatoms. Limnology and Oceanography, 46, 86-94.

Fischer, J., \& Visbeck, M. (1993). Seasonal variation of the daily zooplankton migration in the Greenland Sea. Deep Sea Research Part I: Oceanographic Research Papers, 40, 1547-1557.

Fleming, R. H. (1939). The control of diatom populations by grazing. ICES Journal of Marine Science, 14, 210-227.

Fleminger, A., \& Hulsemann, K. (1977). Geographical range and taxonomic divergence in North Atlantic Calanus (C. helgolandicus, C. finmarchicus, and C. glacialis). Marine Biology, 40, 233-248.

Flierl, G., \& Davis, C. S. (1993). Biological effects of Gulf Stream meandering. Journal of Marine Research, 51, 529-560.

Forsythe, W. C., Rykiel, E. J., Stahl, R. S., Wu, H., \& Schoolfield, R. M. (1995). A model comparison for daylength as a function of latitude and day of year. Ecological Modelling, 80, 87-95.

Franks, P. (2002). NPZ models of plankton dynamics: Their construction, coupling to physics, and application. Journal of Oceanography, 58, 379-387.

Franks, P. J., \& Chen, C. (2000). A 3-D prognostic numerical model study of the Georges Bank ecosystem. Part II: biological-physical model. Deep Sea Research Part II: Topical Studies in Oceanography, 48, 457-482.

Fredrickson, A. G. (1977). Behavior of mixed cultures of microorganisms. Annual Review of Microbiology, 31, 63-87.

Fussmann, G. F., \& Blasius, B. (2005). Community response to enrichment is highly sensitive to model structure. Biology Letters, 1, 9-12.

Gangopadhyay, A., Gawarkiewicz, G., Silva, E. N. S., Monim, M., \& Clark, J. (2019). An observed regime shift in the formation of warm core rings from the Gulf Stream. Scientific Reports, 9, 1-9.

Geider, R. J., Platt, T., \& Raven, J. A. (1986). Size dependence of growth and photosynthesis in diatoms: A synthesis. Marine Ecology Progress Series, 30, 93-104. 
Gentleman, W., Leising, A., Frost, B., Strom, S., \& Murray, J. (2003). Functional responses for zooplankton feeding on multiple resources: A critical review of assumed biological dynamics. Deep Sea Research Part II: Topical Studies in Oceanography, 50, 2847-2875.

Gin, K. Y. H., Guo, J., \& Cheong, H. F. (1998). A size-based ecosystem model for pelagic waters. Ecological Modeling, 112, 53-72.

Gloege, L., McKinley, G. A., Mouw, C. B., \& Ciochetto, A. B. (2017). Global evaluation of particulate organic carbon flux parameterizations and implications for atmospheric pCO2. Global Biogeochemical Cycles, 31, 1192-1215.

Gregg, W. W., Ginoux, P., \& Schopf, P. S. (2003). Phytoplankton and iron: Validation of a global three-dimensional ocean biogeochemical model. Deep Sea Research Part II: Topical Studies in Oceanography, 50, 3143-3169.

Grieve, B. D., Hare, J. A., \& Saba, V. S. (2017). Projecting the effects of climate change on Calanus finmarchicus distribution within the U.S. Northeast Continental Shelf. Scientific Reports, 7, $1-12$.

Guyennon, A., Baklouti, M., Diaz, F., Palmieri, J., Beuvier, J., Lebaupin-Brossier, C., Arsouze, T., Béranger, K., Dutay, J. C., \& Moutin, T. (2015). New insights into the organic carbon export in the Mediterranean Sea from 3-D modeling. Biogeosciences, 12, 7025-7046.

Hales, B., Vaillancourt, R. D., Prieto, L., Marra, J., Houghton, R., \& Hebert, D. (2009). Highresolution surveys of the biogeochemistry of the New England shelfbreak front during Summer, 2002. Journal of Marine Systems, 78, 426-441.

Haney, J. F. (1988). Diel patterns of zooplankton behaviour. Bulletin of Marine Science, 43, 583603.

Hardin, G. (1960). The competitive exclusion principle. Science, 131, 1292-1297.

Hassell, M. P. (1978). The Dynamics of Arthropod Predator-Prey Systems. Princeton University Press.

Haydon, D. (1994). Pivotal assumptions determining the relationship between stability and complexity : An analytical synthesis of the stability-complexity debate. The American Naturalist, $144,14-29$.

Hays, G. C. (2003). A review of the adaptive significance and ecosystem consequences of zooplankton diel vertical migrations. Hydrobiologia, 503, 163-170.

Henson, S. A., Sanders, R., Madsen, E., Morris, P. J., Le Moigne, F., \& Quartly, G. D. (2011). A reduced estimate of the strength of the ocean's biological carbon pump. Geophysical Research Letters, 38, 10-14.

Hernández-León, S., Gómez, M., Pagazaurtundua, M., Portillo-Hahnefeld, A., Montero, I., \& Almeida, C. (2001). Vertical distribution of zooplankton in Canary Island waters: Implications for export flux. Deep Sea Research Part I: Oceanographic Research Papers, 48, 1071-1092.

Holling, C. S. (1959). The components of predation as revealed by a study of small-mammal predation of the European Pine Sawfly. The Canadian Entomologist, 91, 293-320.

Holt, R. D., Grover, J., \& Tilman, D. (1994). Simple rules for interspecific dominance in systems with exploitative and apparent competition. The American Naturalist, 144, 741-771.

Hsu, S. B. (1978). Limiting behavior for competing species. SIAM Journal on Applied Mathematics, 34, 760-763.

Hughes, R. N., \& Croy, M. I. (1993). An experimental analysis of frequency-dependent predation (switching) in the 15-Spined Stickleback, Spinachia spinachia. The Journal of Animal Ecology, 62, 341.

Hutchinson, G. E. (1941). Ecological aspects of succession in natural populations. The American Naturalist, 75, 406-418.

Hutchinson, G. E. (1961). The paradox of the plankton. The American Naturalist, 95, 137-145. 
Ikeda, T. (2014). Respiration and ammonia excretion by marine metazooplankton taxa: synthesis toward a global-bathymetric model. Marine Biology, 161, 2753-2766.

Isla, A., \& Anadon, R. (2004). Mesozooplankton size-fractionated metabolism and feeding off NW Spain during autumn: Effects of a poleward current. ICES Journal of Marine Science, 61, 526-534.

Isla, A., Scharek, R., \& Latasa, M. (2015). Zooplankton diel vertical migration and contribution to deep active carbon flux in the NW Mediterranean. Journal of Marine Systems, 143, 86-97.

Jost, J. L., Drake, J. F., Tsuchiya, H. M., \& Fredrickson, A. G. (1973). Microbial food chains and food webs. Journal of Theoretical Biology, 41, 461-484.

Kempf, A., Floeter, J., \& Temming, A. (2008). Predator-prey overlap induced Holling type III functional response in the North Sea fish assemblage. Marine Ecology Progress Series, 367, 295-308.

Key, T., McCarthy, A., Campbell, D. A., Six, C., Roy, S., \& Finkel, Z. V. (2010). Cell size trade-offs govern light exploitation strategies in marine phytoplankton. Environmental Microbiology, 12, 95-104.

Kiørboe, T., Saiz, E., \& Viitasalo, M. (1996). Prey switching behaviour in the planktonic copepod Acartia tonsa. Marine Ecology Progress Series, 143, 65-75.

Klevjer, T. A., Irigoien, X., Røstad, A., Fraile-Nuez, E., Benítez-Barrios, V. M., \& Kaartvedt., S. (2016). Large scale patterns in vertical distribution and behaviour of mesopelagic scattering layers. Scientific Reports, 6, 19873.

Kwon, E. Y., Primeau, F., \& Sarmiento, J. L. (2009). The impact of remineralization depth on the air-sea carbon balance. Nature Geoscience, 2, 630-635.

Lampert, W. (1989). The adaptive significance of diel vertical migration of zooplankton. Functional Ecology, 3, 21-27.

Last, K. S., Hobbs, L., Berge, J., Brierley, A. S., \& Cottier, F. (2016). Moonlight drives ocean-scale mass vertical migration of zooplankton during the Arctic winter. Current Biology, 26, 244251.

Laufkötter, C., John, J. G., Stock, C. A., \& Dunne, J. P. (2017). Temperature and oxygen dependence of the remineralization of organic matter. Global Biogeochemical Cycles, 31, 10381050 .

Laufkötter, C., Vogt, M., Gruber, N., Aumont, O., Bopp, L., Doney, S. C., Dunne, J. P., Hauck, J., John, J. G., Lima, I. D., Seferian, R., \& Völker, C. (2016). Projected decreases in future marine export production: The role of the carbon flux through the upper ocean ecosystem. Biogeosciences, 13, 4023-4047.

Le Quéré, C., Harrison, S. P., \& Prentice, I. C. (2005). Ecosystem dynamics based on plankton functional types for global ocean biogeochemistry models. Global Change Biology, 11, 20162040.

Leibold, M. A. (1996). A graphical model of keystone predators in food webs: Trophic regulation of abundance, incidence, and diversity patterns in communities. The American Naturalist, $147,784-812$.

Letscher, R. T., Moore, J. K., Teng, Y. C., \& Primeau, F. (2015). Variable C:N:P stoichiometry of dissolved organic matter cycling in the Community Earth System Model. Biogeosciences, 12, 209-221.

Lima, I. D., Lam, P. J., \& Doney, S. C. (2014). Dynamics of particulate organic carbon flux in a global ocean model. Biogeosciences, 11, 1177-1198.

Linder, C. A., Gawarkiewicz, G. G., \& Pickart, R. S. (2004). Seasonal characteristics of bottom boundary layer detachment at the shelfbreak front in the Middle Atlantic Bight. Journal of Geophysical Research: Oceans, 109, 1-9. 
Longhurst, A. R., Bedo, A. W., Harrison, W. G., Head, E. J. H., \& Sameoto, D. D. (1990). Vertical flux of respiratory carbon by oceanic diel migrant biota. Deep Sea Research Part I: Oceanographic Research Papers, 37, 685-694.

Madin, L. P., Horgan, E. F., \& Steinberg, D. K. (2001). Zooplankton at the Bermuda Atlantic Timeseries Study (BATS) station: Diel, seasonal and interannual variation in biomass, 1994-1998. Deep-Sea Research Part II: Topical Studies in Oceanography, 48, 2063-2082.

Marañón, E., Cermeño, P., López-Sandoval, D. C., Rodríguez-Ramos, T., Sobrino, C., Huete-Ortega, M., Blanco, J. M., \& Rodríguez, J. (2013). Unimodal size scaling of phytoplankton growth and the size dependence of nutrient uptake and use. Ecology Letters, 16, 371-379.

Mariani, P., \& Visser, A. W. (2010). Optimization and emergence in marine ecosystem models. Progress in Oceanography, 84, 89-92.

Marra, J., Houghton, R. W., Boardman, D. C., \& Neale, P. J. (1982). Variability in surface chlorophyll a at a shelf-break front (New York Bight). Journal of Marine Research, 40, 575-591.

Marsay, C. M., Sanders, R. J., Henson, S. A., Pabortsava, K., Achterberg, E. P., \& Lampitt, R. S. (2015). Attenuation of sinking particulate organic carbon flux through the mesopelagic ocean. Proceedings of the National Academy of Sciences, 112, 1089-1094.

Martin, J. H., Knauer, G. A., Karl, D. M., \& Broenkow, W. W. (1987). VERTEX: Carbon cycling in the northeast Pacific. Deep Sea Research Part I: Oceanographic Research Papers, 34, 267285.

May, R. M. (1973). Stability and Complexity in Model Ecosystems. Princeton University Press.

McGillicuddy, D. J., McCarthy, J. J., \& Robinson, A. R. (1995a). Coupled physical and biological modeling of the spring bloom in the North-Atlantic: Model formulation and one dimensional bloom processes. Deep Sea Research Part I: Oceanographic Research Papers, 42, 1313-1357.

McGillicuddy, D. J., Robinson, A. R., \& McCarthy, J. J. (1995b). Coupled physical and biological modeling of the spring bloom in the North-Atlantic: 3-dimensional bloom and post-bloom processes. Deep Sea Research Part I: Oceanographic Research Papers, 42, 1359-1398.

Michaels, A. F., \& Silver, M. W. (1988). Primary production, sinking fluxes and the microbial food web. Deep-Sea Research Part I: Oceanographic Research Papers, 35, 473-490.

Moberg, E. A., \& Sosik, H. M. (2012). Distance maps to estimate cell volume from two-dimensional plankton images. Limnology and Oceanography: Methods, 10, 278-288.

Moloney, C. L., \& Fields, J. G. (1991). The size-based dynamics of plankton food webs. I. A simulation model of carbon and nitrogen flows. Journal of Plankton Research, 13, 10031038 .

Moore, J. K., Doney, S. C., Kleypas, J. A., Glover, D. M., \& Fung, I. Y. (2002). An intermediate complexity marine ecosystem model for the global domain. Deep Sea Research Part II: Topical Studies in Oceanography, 49, 403-462.

Moore, J. K., Doney, S. C., \& Lindsay, K. (2004). Upper ocean ecosystem dynamics and iron cycling in a global three-dimensional model. Global Biogeochemical Cycles, 18, 1-21.

Morel, A., \& Bricaud, A. (1981). Theoretical results concerning light absorption in a discrete medium, and application to specific absorption of phytoplankton. Deep Sea Research Part I: Oceanographic Research Papers, 28, 1375-1393.

Moriarty, R., \& O'Brien, T. D. (2013). Distribution of mesozooplankton biomass in the global ocean. Earth System Science Data, 5, 45-55.

Morozov, A. Y. (2010). Emergence of Holling type III zooplankton functional response: Bringing together field evidence and mathematical modelling. Journal of Theoretical Biology, 265, $45-54$.

Murdoch, W. W. (1969). Switching in general predators: Experiments on predator specificity and stability of prey populations. Ecological Monographs, 39, 335-354. 
Murdoch, W. W., Avery, S., \& Smyth, M. E. B. (1975). Switching in predatory fish. Ecology, 56, 1094-1105.

Nissen, C., Vogt, M., Münnich, M., Gruber, N., \& Haumann, F. A. (2018). Factors controlling coccolithophore biogeography in the Southern Ocean. Biogeosciences, 15, 6997-7024.

Ochoa, J., Maske, H., Sheinbaumc, J., \& Candela, J. (2013). Diel and lunar cycles of vertical migration extending to below $1000 \mathrm{~m}$ in the ocean and the vertical connectivity of depthtiered populations. Limnology and Oceanography, 58, 1207-1214.

Oliver, H., Zhang, W. G., Alatalo, P., Chappell, P. D., Hirzel, A., Selden, C. R., Sosik, H. M., Stanley, R. H. R., Zhu, Y., \& McGillicuddy, D. J. (2020). Extraordinary diatom blooms driven by western boundary current instability. Geophysical Research Letters, in prep.

O'Reilly, J. E., \& Busch, D. A. (1984). Phytoplankton primary production on the northwestern Atlantic shelf. Rapp. et Proc.-Verbaux Re'un. Cons. Int. Explor. Mer, 183, 255-268.

Paffenhöfer, G. A. (1984). Food ingestion by the marine planktonic copepod Paracalanus in relation to abundance and size distribution of food. Marine Biology, 80, 323-333.

Pielou, E. C. (1966). The measurement of diversity in different types of biological collections. Journal of Theoretical Biology, 13, 131-144.

Poulin, F. J., \& Franks, P. J. S. (2010). Size-structured planktonic ecosystems: Constraints, controls and assembly instructions. Journal of Plankton Research, 32, 1121-1130.

Prowe, A. E. F., Pahlow, M., Dutkiewicz, S., Follows, M., \& Oschlies, A. (2012). Top-down control of marine phytoplankton diversity in a global ecosystem model. Progress in Oceanography, $101,1-13$.

Putzeys, S., \& Hernández-Leon, S. (2005). A model of zooplankton diel vertical migration off the Canary Islands: Implication for active carbon flux. Journal of Sea Research, 53, 213-222.

Riley, G. A. (1946). Factors controlling phytoplankton populations on Georges Bank. Journal of Marine Research, 6.

Roughgarden, J., \& Feldman, M. (1975). Species packing and predation pressure. Ecology, 56, 489492.

Ryan, J. P., Yoder, J. A., Barth, J. A., \& Cornillon, P. C. (1999). Chlorophyll enhancement and mixing associated with meanders of the shelf break front in the Mid-Atlantic Bight. Journal of Geophysical Research: Oceans, 104, 23479-23493.

Sabine, C. L., Feely, R. A., Gruber, N., Key, R. M., Lee, K., Bullister, J. L., Wanninkhof, R., Wong, C. S., Wallace, D. W. R., Tilbrook, B., Millero, F. J., Peng, T. H., Kozyr, A., Ono, T., \& Rios, A. F. (2004). The oceanic sink for anthropogenic CO2. Science, 305, 367-371.

Schminke, H. K. (2007). Entomology for the copepodologist. Journal of Plankton Research, 29, i149-i162.

Shannon, C. E. (1948). A mathematical theory of communication. Mobile Computing and Communications Review, 5, 3-55.

Sieburth, J. M., Smetacek, V., \& Jurgen, L. (1978). Pelagic ecosystem structure : Heterotrophic compartments of the plankton and their relationship to plankton size fractions. Limnology and Oceanography, 23, 1256-1263.

Siegel, D. A., Buesseler, K. O., Behrenfeld, M. J., Benitez-Nelson, C. R., Boss, E., Brzezinski, M. A., Burd, A., Carlson, C. A., D’Asaro, E. A., Doney, S. C., Perry, M. J., Stanley, R. H. R., \& Steinberg, D. K. (2016). Prediction of the export and fate of global ocean net primary production: The EXPORTS science plan. Frontiers in Marine Science, 3, 1-10.

Siegel, D. A., Buesseler, K. O., Doney, S. C., Sailley, S. F., Behrenfeld, M. J., \& Boyd, P. W. (2014). Global assessment of ocean carbon export by combining satellite observations and food-web models. Global Biogeochemical Cycles, 28, 181-196. 
Slobodkin, L. B. (1980). Growth and Regulation of Animal Populations (2nd ed.). Dover Publications.

Smith, S. L., Vallina, S. M., \& Merico, A. (2016). Phytoplankton size-diversity mediates an emergent trade-off in ecosystem functioning for rare versus frequent disturbances. Scientific Reports, 6, 34170 .

Son, S. H., \& Wang, M. (2015). Diffuse attenuation coefficient of the photosynthetically available radiation $\mathrm{Kd}(\mathrm{PAR})$ for global open ocean and coastal waters. Remote Sensing of Environment, 159, 250-258.

Song, C., Barabás, G., \& Saavedra, S. (2019). On the consequences of the interdependence of stabilizing and equalizing mechanisms. American Naturalist, 194, 627-639.

Sosik, H. M., Futrelle, J., Brownlee, E. F., Peakcock, E., Crockford, T., \& Olson, R. J. (2020). Ifcb-analysis.

Sosik, H. M., \& Olson, R. J. (2007). Automated taxonomic classification of phytoplankton sampled with imaging-in-flow cytometry. Limnology and Oceanography: Methods, 5, 204-216.

Steele, J. H. (1974). The Structure of Marine Ecosystems. Harvard University Press.

Steinberg, D. K., Carlson, C. A., Bates, N. R., Goldthwait, S. A., Madin, L. P., \& Michaels, A. F. (2000). Zooplankton vertical migration and the active transport of dissolved organic and inorganic carbon in the Sargasso Sea. Deep-Sea Research Part I: Oceanographic Research Papers, 47, 137-158.

Steinberg, D. K., Carlson, C. A., Bates, N. R., Johnson, R. J., Michaels, A. F., \& Knap, A. H. (2001). Overview of the US JGOFS Bermuda Atlantic Time-series Study (BATS): A decadescale look at ocean biology and biogeochemistry. Deep-Sea Research II: Topical Studies in Oceanography, 48, 1405-1447.

Steinberg, D. K., Goldthwait, S. A., \& Hansell, D. A. (2002). Zooplankton vertical migration and the active transport of dissolved organic and inorganic nitrogen in the Sargasso Sea. Deep-Sea Research I: Oceanographic Research Papers, 49, 1-17.

Steinberg, D. K., \& Landry, M. R. (2017). Zooplankton and the ocean carbon cycle. Annual Review of Marine Science, 9, 413-444.

Strom, S. L., \& Loukos, H. (1998). Selective feeding by protozoa: Model and experimental behaviors and their consequences for population stability. Journal of Plankton Research, 20, 831-846.

Strom, S. L., Miller, C. B., \& Frost, B. W. (2000). What sets lower limits to phytoplankton stocks in high-nitrate, low-chlorophyll regions of the open ocean? Marine Ecology Progress Series, 193, 19-31.

Takahashi, K., Kuwata, A., Sugisaki, H., Uchikawa, K., \& Saito, H. (2009). Downward carbon transport by diel vertical migration of the copepods Metridia pacifica and Metridia okhotensis in the Oyashio region of the western subarctic Pacific Ocean. Deep-Sea Research I: Oceanographic Research Papers, 56, 1777-1791.

Tanioka, T., \& Matsumoto, K. (2018). Effects of incorporating age-specific traits of zooplankton into a marine ecosystem model. Ecological Modelling, 368, 257-264.

Teramoto, E. I., Kohkichi, K., \& Nanako, S. (1979). Switching effect of predation on competitive prey species. Journal of Theoretical Biology, 79, 303-315.

Thingstad, T. F. (2000). Elements of a theory for the mechanisms controlling abundance, diversity, and biogeochemical role of lytic bacterial viruses in aquatic systems. Limnology and Oceanography, 45, 1320-1328.

Thorton, P. E., Doney, S. C., Lindsay, K., Moore, J. K., Mahowald, N., Randerson, J. T., Fung, I., Lemarque, J. F., Feddema, J. J., \& Lee, Y. H. (2009). Carbon-nitrogen interactions regulate climate-carbon cycle feedbacks: Results from an atmosphere-ocean general circulation model. Biogeosciences, 6, 2099-2120. 
Tilman, D. (1977). Resource competition between plankton algae : An experimental and theoretical approach. Ecology, 58, 338-348.

Tilman, D. (1982). Resource Competition and Community Structure. Princeton University Press.

Tilman, D. (2001). Functional diversity. Encyclopedia of biodiversity, 3, 109-120.

Vallina, S. M., Cermeno, P., Dutkiewicz, S., Loreau, M., \& Montoya, J. M. (2017). Phytoplankton functional diversity increases ecosystem productivity and stability. Ecological Modelling, 361, 184-196.

Vallina, S. M., Follows, M. J., Dutkiewicz, S., Montoya, J. M., Cermeno, P., \& Loreau, M. (2014a). Global relationship between phytoplankton diversity and productivity in the ocean. Nature Communications, 5.

Vallina, S. M., \& Le Quéré, C. (2011). Stability of complex food webs: Resilience, resistance and the average interaction strength. Journal of Theoretical Biology, 272, 160-173.

Vallina, S. M., Ward, B. A., Dutkiewicz, S., \& Follows, M. J. (2014b). Maximal feeding with active prey-switching: A kill-the-winner functional response and its effect on global diversity and biogeography. Progress in Oceanography, 120, 93-109.

Verdy, A., Follows, M. J., \& Flierl, G. (2009). Optimal phytoplankton cell size in an allometric model. Marine Ecology Progress Series, 379, 1-12.

Vinogradov, M. E. (1962). Feeding of the deep-sea zooplankton. Rapp. Rv Réun Cons. Perm. Int. Explor. Mer., 153, 114-119.

Wallace, M. I., Cottier, F. R., Brierley, A. S., \& Tarling, G. A. (2013). Modelling the influence of copepod behaviour on faecal pellet export at high latitudes. Polar Biology, 36, 579-592.

Ward, B. A., Dutkiewicz, S., \& Follows, M. J. (2014). Modelling spatial and temporal patterns in size-structured marine plankton communities: Top-down and bottom-up controls. Journal of Plankton Research, 36, 31-47.

Ward, B. A., Dutkiewicz, S., Jahn, O., \& Follows, M. J. (2012). A size-structured food-web model for the global ocean. Limnology and Oceanography, 57, 1877-1891.

Ward, B. A., \& Follows, M. J. (2016). Marine mixotrophy increases trophic transfer efficiency, mean organism size, and vertical carbon flux. Proceedings of the National Academy of Sciences, 113, 2958-2963.

Wroblewski, J. S. (1977). A model of phytoplankton bloom formation during variable Oregon upwelling. Journal of Marine Research, 35, 357-394.

Zhang, W. G., \& Gawarkiewicz, G. G. (2015). Dynamics of the direct intrusion of Gulf Stream ring water onto the Mid-Atlantic Bight shelf. Geophysical Research Letters, 42, 7687-7695.

Zhang, W. G., Gawarkiewicz, G. G., McGillicuddy, D. J., \& Wilkin, J. L. (2011). Climatological mean circulation at the New England shelf break. Journal of Physical Oceanography, 41, $1874-1893$.

Zhang, W. G., McGillicuddy, D. J., \& Gawarkiewicz, G. G. (2013). Is biological productivity enhanced at the New England shelfbreak front? Journal of Geophysical Research: Oceans, 118, $517-535$.

Zhang, X., \& Dam, H. G. (1997). Downward export of carbon by diel migrant mesozooplankton in the central equatorial Pacific. Deep Sea Research II: Topical Studies in Oceanography, 44, $2191-2202$.

Zhou, M. (2006). What determines the slope of a plankton biomass spectrum? Journal of Plankton Research, 28, 437-448. 